\title{
Feasibility of Designing Aerogel Process for Targeted Thermal Conductivity by Controlling Porosity
}

\author{
A Dissertation \\ Presented to
}

the faculty of the School of Engineering and Applied Science

University of Virginia

in partial fulfillment

of the requirements for the degree

Doctor of Philosophy

by

Jong Ho Sonn

August 2017 


\title{
APPROVAL SHEET
}

\author{
This Dissertation \\ is submitted in partial fulfillment of the requirements \\ for the degree of \\ Doctor of Philosophy
}

Author Signature:

This Dissertation has been read and approved by the examining committee:

Advisor: Pamela M. Norris

Committee Member: Patrick E. Hopkins

Committee Member: Gavin T. Garner

Committee Member: David Green

Committee Member: Elizabeth J. Opila

Committee Member:

Accepted for the School of Engineering and Applied Science:

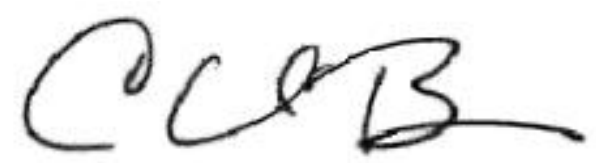

Craig H. Benson, School of Engineering and Applied Science

August 2017 


\section{Abstract}

For decades, there have been global issues of environmental pollution and energy availability. Aerogels have been steadily suggested as alternative thermal insulation materials in various applications to aid in solutions to these issues. This effort has resulted in some aerogel products being commercialized and available on the market. The size of the aerogel market in the thermal insulation industry has been steadily increasing over time. This market for thermal insulation also continues to find new products and upgrade the aerogel products to satisfy the needs from both the suppliers and customers. In order for aerogel insulation to stay competitive, development of an aerogel production process that has high feasibility for controlling thermal conductivity is undoubtedly important. Successful development of the aerogel production process should also enable simple integration of the developed process with a conventional material process for hybrid aerogel products. In addition, the new process should contribute to reducing the production cost.

A good understanding of how thermal conduction occurs in aerogel is crucial to guide development of a new aerogel production process. In this research, the sensitivity of thermal conductivity in aerogel materials to the porosity is investigated. This investigation shows that higher porosity results in lower thermal conductivity for aerogels with porosities of $80 \%$ or greater. It also demonstrates that the production process of aerogel insulation should be continuously optimized even when the result of optimization only reduced thermal conductivity of the product by less than $1.0 \mathrm{~mW} / \mathrm{m} \cdot \mathrm{K}$.

Motivated by the investigation results, a new sol-gel process, called the direct-contact method (DCM, US Provisional Patent Application Serial No. 62/486,057 filed April 17, 2017) is developed and introduced in this research. This new process replaces the conventional pre-hydrolysis step in the two step sol-gel process. By employing this method, silica alkoxide is pre-hydrolyzed by a special combination of catalysts without solvent and heating. As a result, clear and pre-hydrolyzed silica sol is produced and directly available for further gelation steps within a few hours at ambient temperature. The DCM also yields a sol with higher concentration of silica than that obtained from a conventional method, so the density range of the final gel product is extended by approximately 3 times. This advantage increases feasibility of the entire sol-gel process with regards to combining with any existing material processes. The production costs of silica aerogel insulation can be remarkably reduced as the time and energy consumed by the DCM is much less compared to the conventional two step process. With these advantages, DCM still produces a pre-hydrolyzed sol 
comparable with a functional silane, which is commercially available at a much higher price than TEOS.

By taking advantage of the DCM, study on how porosity and the porous structure of silica aerogel is affected by the parameters of its production process is pursued. This study demonstrates successful integration of the DCM with the two step sol-gel route. The DCM enables simplification of the entire sol-gel process and still produces monolithic silica aerogels with over $90 \%$ reproducibility in terms of gelation time, densities, and main pore size. It also shows high feasibility for tuning the synthetic conditions to obtain a desired target property of the produced silica aerogel including porosity.

In addition, studies on the effects of target density on porosity of yttria aerogels and organic aerogels are pursued and their results are introduced. Both types of aerogel monoliths were produced from modified sol-gel processes and characterized in order to outline how target density is related to apparent density and skeletal density. The study for yttria aerogel aids in recognizing the importance of controlling skeletal density of the produced yttria aerogel in order to obtain a desired porosity by strategizing the sol-gel process. Through the study of organic aerogel, it is recognized that the porosity has high sensitivity to the apparent density, as it rapidly increased when apparent density was decreased. These results aid in defining the range of target density for future research on organic aerogel in order to understand the porosity change as well as the porosity.

This research finally presents a newly developed experimental method for preparation of aerogel samples for thermal conductivity measurements using the established $3 \oplus$ method. The new method requires installation of a wire set inside a monolithic aerogel so that it can play the role of the 4 connectors needed for the $3 \omega$ method. These experimental $3 \omega$ results show that thermal conductivities decreased with increased porosities, thus agreeing well with the discussion and model presented in this research. These results also demonstrate that the wire set implantation can be a reliable method that simplifies sample preparation for $3 \curvearrowleft$ measurements in aerogel materials. 


\section{Acknowledgements}

First, I praise God of Ebenezer for He is the Ruler of this universe and He has let me look into very small part of His creation so that I could study and appreciate it.

I sincerely thank my advisor, Dean Norris, for letting me continue to work on aerogel at her research lab and providing me every milestone of the way with unthinkable trust and support as well as guidance, which I didn't deserve by big margin. Thanks to Pam's encouragement at every moment I stumbled due to unforeseen issues in my research, I could keep on walking forward and made achievement way more than I can only with my capability. I also want to give Pam another thanks separately since she made me think about directing my next destiny to a teaching position.

I thank my advisory committee, all of whom have been very generous with their time and expertise: Patrick Hopkins, David Green, Gavin Garner, and Elizabeth Opila. I think each of you can identify the huge swaths of this work that I owed directly to classes and conversations I've had with you over the years. I want to deeply appreciate the unending supports from Dr. Park, Professor Lim, Dr. Suh, and Mr. Yoon who were all my great mentors in the past.

I acknowledge my colleagues at Aerogel Research Lab and Nanosclae Heat Transfer Lab for many hours of communication and support for my research as well as proposing many possibilities, some of which actually have been visualized and taken into real experiments for my final works. Irreplaceable assist from Bo Hosticka provided me major breakthrough in characterization study and I would never forget that Bo gave me a ride on his beloved antique car - I wish I remembered the model name of it. It has been and will be the coolest ride I ever receive in my life. I want to say specially thank you to Casey Bauer, Chris Saltonstall, and Christina Pappas, who began this journey together and stayed together at the classes and worked on assignments with a good team spirit. I wish them best of luck as all of them are in places for their lives after graduation.

My family specially warrants thanks for any success I've had to this point in my life. My father and mother, both have been already Doctor over 40 years in their professions their loves to me have been always unthinkable and they themselves have been always my standpoints in my pursuance of this study. Seung-Hyun, my only sister, Dr. Sonn, only brother have been my comforters. Mr. Choi and Mrs. Kang, who are my brother- in-law and sister-in-law have been my supporters. HuiJo and Young-Min are always my loving niece and nephew and I have been happy to see you grow 
over time. My brothers and sisters of Dongyuk in Christ, I know that I owed you big time since they have been praying for me since we met

Finally, Mihee and Youngush, I have no proper words to express my grateful heart to you sufficiently. Thank you, and thank you. 


\section{Table of Contents}

Aerogel

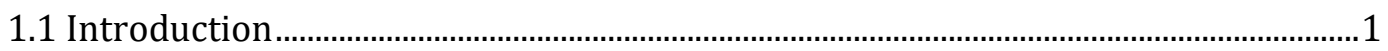

1.1.1 Solution-sol-gel process ............................................................................. 1

1.1.2 Supercritical drying process......................................................................... 5

1.1.3 Ambient drying process .............................................................................. 9

1.2 Historical Background .................................................................................................. 10

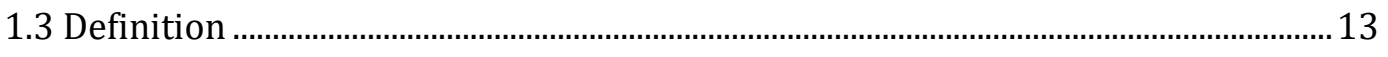

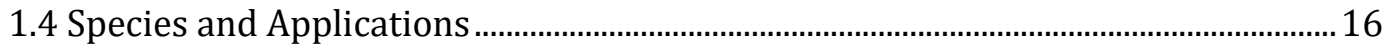

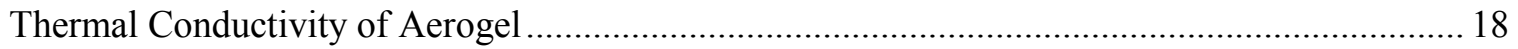

2.1 Thermal Conductivity of Amorphous Materials …….................................................. 18

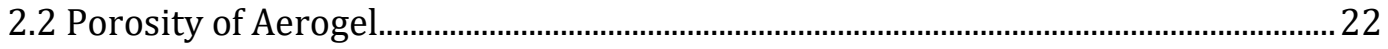

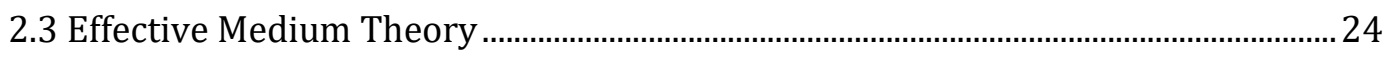

2.4 Modified Model for Porous Materials with Advantage ....................................................27

2.5 Sensitivity of the Thermal Conductivity of Aerogel to its Porosity............................. 31

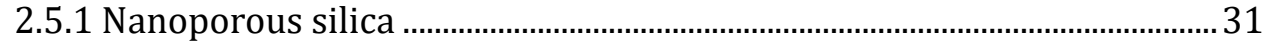

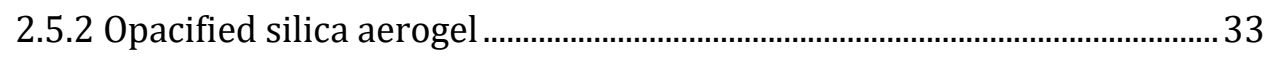

2.5.3 Hybrid organic-inorganic aerogel ................................................................... 37

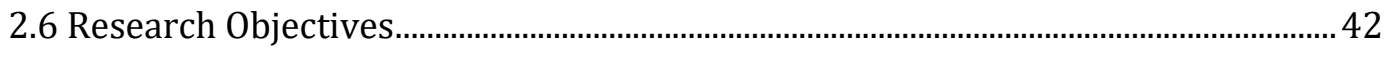

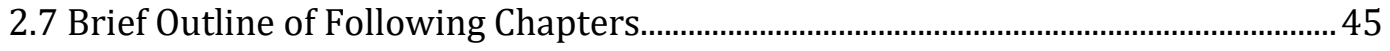

New Sol-Gel Route for Silica Aerogel Synthesis: Direct-Contact Method (DCM) …................ 47

3.1 Motivation for Process Development................................................................................ 47

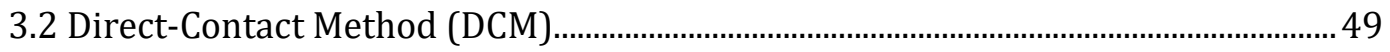

3.2.1 Description of the developed process ............................................................ 49 
3.2.3 Quantitative definition of each composition.................................................53

3.2.4 Observation of the DCM process........................................................................ 54

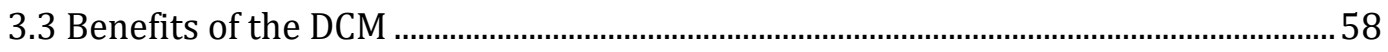

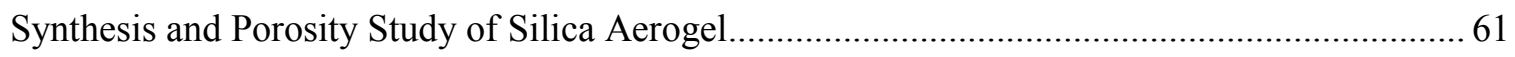

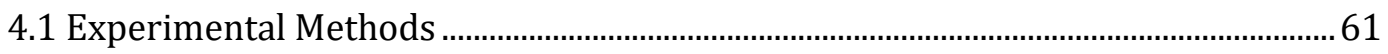

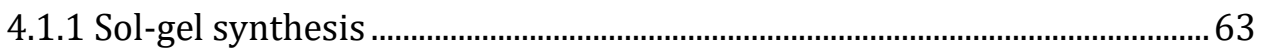

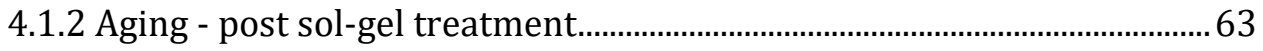

4.1.3 Supercritical drying process and low temperature calcination................65

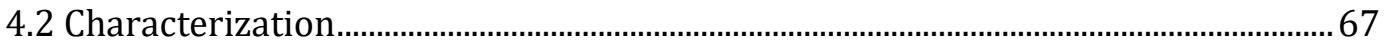

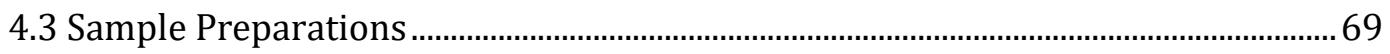

4.3.1 Variations in target density and porosity of "standard samples" via gelation time ................................................................................................. 70

4.3.2 Variations in solvent types of "standard samples" via changing

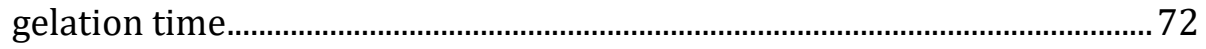

4.3.3 Variations in the catalytic combination in the DCM .................................74

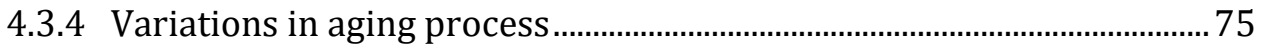

4.4 Results and Discussion .............................................................................................. 76

4.4.1 Effects of target density on gelation time of monolithic silica

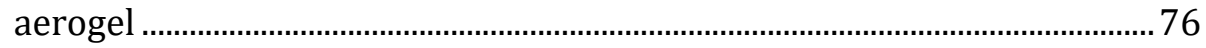

4.4.2 Effects of solvent types on gelation time of monolithic silica

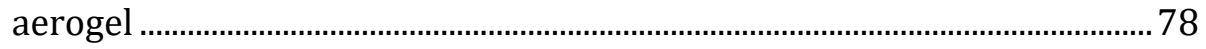

4.4.3 Effects of apparent density on transparency of monolithic silica aerogel

4.4.4 Effects of target density on apparent density and skeletal

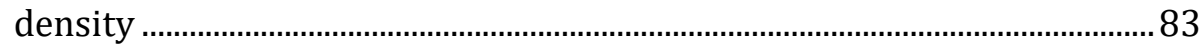

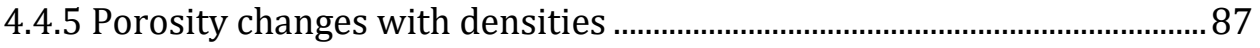

4.4.6 Discussion of morphological analysis from SEM pictures.......................... 89

4.4.7 Effects of processing conditions on porosity …..............................................90

4.5 Conclusions 


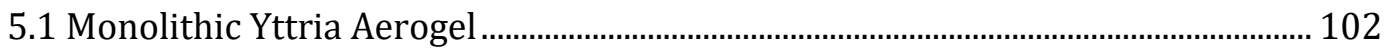

5.1.1 Experimental method ................................................................................. 103

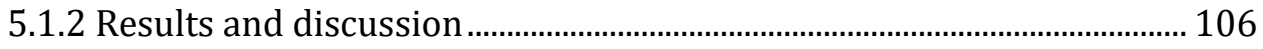

5.2 Synthesis and porosity study of organic aerogel....................................................... 112

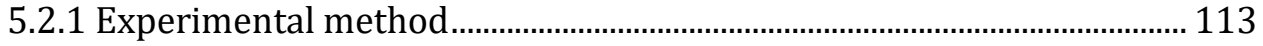

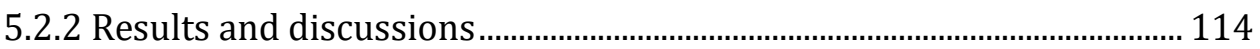

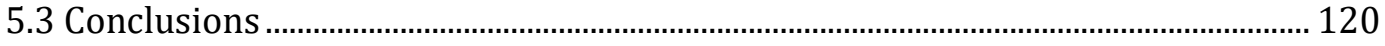

Thermal Conductivity of Monolithic Aerogel Determined with Implanted Wire Set by $3 \omega$ Method 122

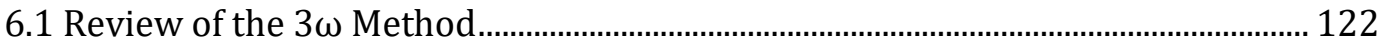

6.2 Customized Sample Preparation for 3๑ Measurement.......................................... 124

6.3 Determining a Heat Diffusion Equation .................................................................... 127

6.4 Sample Preparation and Anticipated Results .............................................................. 128

6.4.1 Preparation of silica aerogels with wire sets.............................................. 128

6.4.2 Preparation of organic aerogels with wire sets......................................... 130

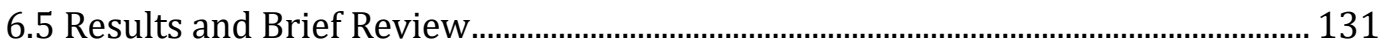

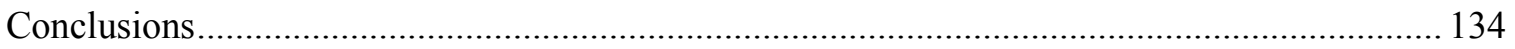

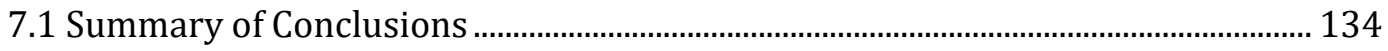

7.2 Suggested Future Studies................................................................................................ 138

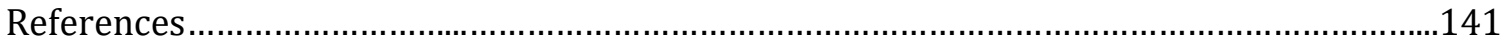




\section{List of Figures}

Figure 1-1. Illustration of a standard sol-gel process. ......................................................................... 2

Figure 1-2. Comparison of required pressures and temperatures for supercritical drying $\left(\mathrm{CO}_{2}\right.$ vs alcohol) [17].

Figure 1-3. First large-scale production unit for silica aerogel in Boston. Alcohol storage tanks in foreground (left). Three of the autoclaves used to dry the gels (right) [24].

Figure 1-4. The produced aerogel is removed from the autoclave (left) and moved in a container (right). The first aerogel product was a mixture of randomly shaped chunks and powders and it was crashed into powders with relatively uniform size. The final product weighed only 3-5 pounds per cubic foot [24].

Figure 1-5. Examples of pore size distributions of ambiently dried silica aerogel (square, [53]) and supercritically dried silica aerogel (diamond, Aerogel Research Lab at University of Virginia).

Figure 1-6. Complex aerogel pore structure describing macro-, meso-, and micro-porosity in addition to surface silanol groups of a "standard" un-modified silica aerogel [54]..................15

Figure 1-7. Application fields of aerogel products [58].................................................................16

Figure 2-1. Debye approximation to the dispersion (black line) and definition of group velocity. Where $a$ is the interatomic spacing.

Figure 2-2 Schematic view from a cross section of the bulk density and the skeletal density of aerogel structure. The blue parts are the solid back bone (skeletal frame) and white parts between the skeletal frame are the pore spaces considered to be the pore volume.................23

Figure 2-3. Sensitivity of thermal conductivity to the atomic density of Si and 0 for hydrogensilsesquioxane films (solid circles labeled by FOx and XLK) and various forms of $\mathrm{SiO}_{2}$. This data was taken and replotted from reference [73]. For comparison, data for carbon aerogels (filled triangles), $\mathrm{SiO}_{2}$ (open circles), porous Vycor (diamonds), xerogel (open squares), and the lattice conductivity of silica aerogel (open triangles) are included. Data for $\mathrm{SiO}_{2}$ are from the literatures $[83,84,85,86,87] .$. The dashed line is the differential effective medium (DEM) theory [73].

Figure 2-4. Effects of atomic density of $\mathrm{Si}$ and $\mathrm{O}$ on thermal conductivity for the aerogel and calcined-aerogel films along with bulk $\mathrm{SiO}_{2}$ [84], a sputtered $\mathrm{SiO}_{2}$ thin film [73], other porous silica materials (XLK and FOx) [73], and bulk $\mathrm{SiO}_{2}$ aerogels [91, 92]. Predictions from the theoretical "porous minimum limit" to the thermal conductivity of $\mathrm{SiO}_{2}$ (solid line - Eq. 2.13) [88].

Figure 2-5. Thermal conductivities of silica aerogels reported with below $0.220 \mathrm{~g} / \mathrm{cm}^{3}$ of apparent densities $[87,93,94,95]$. The porous minimum limit (solid line) and the DEM theory (dashed line) were replotted from reference [88] by assuming the linear relationship between the atomic density of $\mathrm{Si}$ and $\mathrm{O}$ and the apparent density. 30 
Figure 2-6. Summary of sensitivity of thermal conductivity to porosity for $L 0 / R p=0.0,0.1$, 0.5 , and 1.0 in the present nanoporous model considered by Sumirat et al. and comparison with data experimentally reported. [96]. Porous xerogel-a is sintered silica xerogel film and porous xerogel-b is silica xerogel film made with a mixture of ethanol and ethylene glycol solvents [97]. Porous xerogel-c is a $\mathrm{SiO}_{2}$-capped xerogel film [86] and MSSQ is a porous organosilicate film formed by templated vitrification of copolymers consisting of polymeric matrix material of methylsilsesquioxane and porogens [98]. 32

Figure 2-7. Illustration of sensitivity of thermal conductivity of opacified monolithic silica aerogel to apparent density (a) and skeletal density (b) at room temperature. It was arranged and plotted from the data originally presented in reference [92]. The data labels indicate weight pecentage of the opacifier that is carbon nanofiber $(\mathrm{cn})$ 35

Figure 2-8. Sensitivity of thermal conductivity to porosity of opacified monolithic silica aerogel. It was arranged and plotted from the data originally presented in reference [92]. The data labels indicate weight pecentage of the opacifier that is carbon nanofiber $(\mathrm{cn})$. The eror bars for 15,13 , and $10 \mathrm{wt} \% \mathrm{cn}$ are smaller than the data markers 36

Figure 2-9. Hydrolysis and condensation route of the two-step sol-gel process for MTMS hybrid silica alcogel [106].

Figure 2-10. Illustration of sensitivity of thermal conductivity of MTMS hybrid silica aerogel to apparent density (a), skeletal density (b), and porosity (c) at room temperature. The sample aerogels prepared from the molar ratio of $\mathrm{MeOH} / \mathrm{MtMS}(\mathrm{M})=7$ are marked as red dots, $M=14$ as brown dots, $M=21$ as light green dots, $M=28$ as violet dots, and $M=35$ as light blue dots (arranged and plotted from Nadargi et al. 's work [66]). 40

Figure 2-11. U.S. aerogel market revenue by end-use, 2012-2022, The data were taken from Aerogel market by raw material (Clark, Allied Market Research, 2016 [107]). 42

Figure 3-1. The three main reaction steps occurring in the two step sol-gel process of alkoxysilanes: (1) hydrolysis of an alkoxy group produces silanol groups and the respective alcohol; (2a) condensation of two silanol bearing species; and (2b) mixed condensation of a silanol group and an alkoxy group bearing species [108]. 48

Figure 3-2. (a) Schematic comparison of conventional pre-hydrolysis and the direct-contact method (DCM) hydrolysis. (b) Example of the two step sol-gel process by employing the DCM and resultant products. 50

Figure 3-3. pH and temperature profile of the hydrolysis reaction of TEOS by the directcontact method (DCM) when nitric acid and acetic acid catalyzed the hydrolysis. The molar ratio of TEOS : water : nitric acid : acetic acid is $1: 4.83: 1.0 \times 10^{-3}: 3.5 \times 10^{-2}$, respectively...55

Figure 3-4. pH and temperature profile of the hydrolysis reaction of TEOS by the direct contact method (DCM) when sulfuric acid and acetic acid catalyzed the hydrolysis. The molar ratio of TEOS : water : nitric acid : acetic acid is $1: 4.83: 1.2 \times 10^{-3}: 3.5 \times 10^{-2}$ respectively.....57

Figure 4-1. Procedure for production of silica aerogel including the DCM process in the solgel process by the nitric acid-acetic acid combination (N-A comb.). Also includes the optional

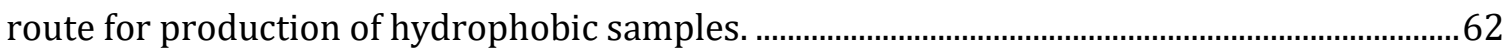

Figure 4-2. Schematic diagram of the supercritical drying unit for the COLD method. .65 
Figure 4-3. Temperature and pressure profiles of $\mathrm{CO}_{2}$ supercritical drying process (the COLD

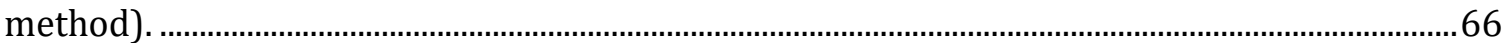

Figure 4-4. AccuPyc II 1340 gas pycnometer at the Aerogel Research Lab (AeRL)..................68

Figure 4-5. Effects of target density on gelation time for the monolithic silica aerogel produced by nitric acid - acetic acid combination for the DCM during sol-gel synthesis (standard process). The three additional samples for the gelation time investigation are $2.0 / 8.0,3.5 / 6.5$, and 7.0/3.0 (highlighted in yellow). The uncertainties are $\pm 5 \%$ and $\pm 2 \%$ in target density and gelation time, respectively 76

Figure 4-6. Effects of target density on gelation time for the monolithic silica aerogel produced by sulfuric acid - acetic acid combination for the DCM during the sol-gel synthesis. The uncertainties are $\pm 5 \%$ and $\pm 2 \%$ in target density and gelation time respectively.........77

Figure 4-7. Transparency comparison of monolithic silica aerogel based on the apparent density (prepared by the standard process). The apparent densities of each aerogel from the left are $0.0529,0.1244,0.1285,0.1733$, and $0.2188 \mathrm{~g} / \mathrm{cm}^{3}$, respectively. The sample IDs from the left are $1.5 / 8.5,2.5 / 7.5,3.0 / 7.0,4.0 / 6.0$, and 5.0/5.0. 79

Figure 4-8. Effects of apparent density $(\mathrm{g} / \mathrm{cm} 3)$ on pore size distributions of monolithic silica aerogels prepared by the standard process (the nitric acid - acetic acid combination for the DCM). The sample IDs from the lowest apparent density are 1.5/8.5, 2.5/7.5, 3.0/7.0, 4.0/6.0, and 5.0/5.0, respectively. .80

Figure 4-9. Effects of target density on actual apparent density of monolithic silica aerogel prepared by the standard process (the nitric acid - acetic acid combination for the DCM). The left value of the data label is the target density $\left(\mathrm{g} / \mathrm{cm}^{3}\right)$ and the right one is the apparent density $\left(\mathrm{g} / \mathrm{cm}^{3}\right)$ for each sample. The uncertainties are $\pm 5 \%$ and $\pm 0.19 \%$ in target density and apparent density, respectively. 83

Figure 4-10. Effects of target density on skeletal density of monolithic silica aerogel (standard process). The left value of the data label is the target density $\left(\mathrm{g} / \mathrm{cm}^{3}\right)$ and the right one is the skeletal density $\left(\mathrm{g} / \mathrm{cm}^{3}\right)$ for each sample. The uncertainties are $\pm 5 \%$ and $\pm 0.06 \%$ in target density and skeletal density respectively. 85

Figure 4-11. Porosity distributions for different target densities (left) and apparent densities of silica aerogels prepared from the standard process. The uncertainties are $\pm 5 \%, \pm 0.19 \%$, and $\pm 0.25 \%$ in target density, apparent density, and gelation time respectively.. .86

Figure 4-12. SEM photographs of $1.5 / 8.5$, which has a low apparent density $\left(0.0529 \mathrm{~g} / \mathrm{cm}^{3}\right)$ and a high skeletal density $\left(2.0106 \mathrm{~g} / \mathrm{cm}^{3}\right)$. The monolithic silica aerogel was prepared by the standard process (magnification: left 1: 50,000, right 1:250,000). . .88

Figure 4-13. SEM photographs of 5.0/5.0, which has a high apparent density $\left(0.2188 \mathrm{~g} / \mathrm{cm}^{3}\right)$ and a low skeletal density $\left(1.7226 \mathrm{~g} / \mathrm{cm}^{3}\right)$. The monolithic silica aerogel was prepared by the standard process (magnification: left 1: 50,000, right 1: 250,000). 89

Figure 4-14. Effects of solvent types in gelation step on pore size distributions of monolithic silica aerogels. The pre-hydrolyzed sol was prepared by the standard DCM (nitric acid - acetic acid combination). 93 
Figure 4-15. Effects of adding water in the gelation step on the pore size distributions of monolithic silica aerogels. The pre-hydrolyzed sol was prepared by the standard DCM (nitric acid - acetic acid combination). 95

Figure 4-16. Change in pore size distribution of silica aerogel synthesized by sulfuric acid acetic acid combination using the DCM.

Figure 4-17. Water droplet on the hydrophobic silica aerogel (left) and SEM photographs of

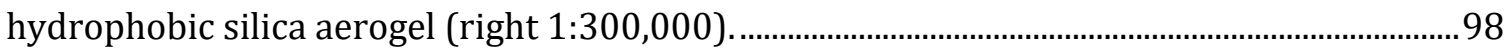

Figure 4-18. Change in pore size distribution of silica aerogel with hydrophobicity. .99

Figure 5-1. The deprotonation and poly-condensation of reaction mechanism for $\mathrm{Y}_{2} \mathrm{O}_{3}$ alcogel synthesis. Chemical equations are based on Reference [121]. 105

Figure 5-2. Summary of the sol-gel investigation and resultant gel states of yttria alcogel prepared by yttrium hexahychloride and propylene oxide in alcoholic solvent environment. 106

Figure 5-3. Photograph of the synthesized yttria alcogel (left) and aerogel (right) 108

Figure 5-4. Change of the porosity values with the apparent densities (a) and the skeletal densities (b) of the monolithic yttria aerogels. 109

Figure 5-5. Effects of the target density change on the pore size distribution of the produced yttria aerogels.

Figure 5-6. Photograph of the produced organic aerogel monoliths. Note that the color differences between the samples come from the different apparent densities. From left to right, the apparent density of each aerogel is $0.237,0.209,0.130,0.113$, and $0.087 \mathrm{~g} / \mathrm{cm}^{3}$, respectively. 114

Figure 5-7. Stages of formation of resole resin (base-catalyzed phenol-formaldehyde resin) for the base-catalyzed PF gel synthesis. The image was taken from Reference [134] 115

Figure 5-8. Change of the porosity values with the apparent densities (a) and the skeletal densities (b) of the monolithic organic aerogels.

Figure 5-9. Effects of the target density change on the pore size distribution of the produced organic aerogels. 118

Figure 6-1. Depiction of simplified 3 $\omega$. Modified from the reference [140]. The monolithic aerogel sample is connected to the 4 -wire set by the 4 wires implanted in the sample...... 123

Figure 6-2. Schematic diagram of procedure for sample aerogel preparation for $3 \omega$ measurement.

Figure 6-3. Schematic diagram of the 3-omega set and the connected monolithic aerogel sample with the implanted wire set (Modified from the reference [140]). 126 


\section{List of Tables}

Table 1-1. Critical constants of fluids used in supercritical extraction......................................... 7

Table 2-1. List of kinetic diameters and atomic diameters of inert gases $[68,69,70]$. The atomic diameters are only available as calculated values except for the value for nitrogen. The atomic diameter of nitrogen listed in this table is a measured value [69]. 25

Table 2-2. Example of developing aerogel process by screening sol-gel parameters with regards to best thermal insulation performance for scale up to a pilot plant (Aspen Aerogels, Inc.). Note that the process (yellow-highlighted) that produced TEHY-1 was selected as the optimized production process because its thermal conductivity is the lowest one among other candidates. The next lowest one, TEHY-2, has higher thermal conductivity only by $0.7 \mathrm{~mW}$ $/ \mathrm{m} \cdot \mathrm{K}$. but not selected. ( ${ }^{\mathrm{a}}$ : hydrolysis time to prepare a sol, ${ }^{\mathrm{b}}$ : apparent density, ${ }^{\mathrm{c}}$ : thermal conductivity). .43

Table 3-1. Investigation results on optimized relative molar ratio of involved compositions in the DCM.

Table 3-2. List of characteristic differences between the conventional method and DCM for pre-hydrolysis in silica sol-gel route. 59

Table 4-1. List of reagents used for the silica aerogel production process. 64

Table 4-2. List of samples of monolithic silica aerogels and their processing conditions prepared by the standard process (the nitric acid - acetic acid combination for the DCM catalyst). The three additional samples for the gelation time investigation are 2.0/8.0, 3.5/6.5, and 7.0/3.0 (highlighted in yellow).

Table 4-3. List of samples of monolithic silica aerogels prepared by different solvent type. All samples were produced at the target density of $0.0582 \mathrm{~g} / \mathrm{cm}^{3}$ so $3.0 \mathrm{ml}$ of the pre-hydrolyzed sol produced by the standard DCM was used to prepare each samples.

Table 4-4. List of samples of monolithic silica aerogels and their processing conditions produced by different catalytic combination of sulfuric acid - acetic acid for the DCM..........73

Table 4-5. List of samples of hydrophilic and hydrophobic monolithic silica aerogels and their aging process. Samples were produced with a target density of $0.0582 \mathrm{~g} / \mathrm{cm}^{3} \mathrm{so} 3.0 \mathrm{ml}$ of the pre-hydrolyzed sol produced by the standard DCM was used to prepare each sample..........75

Table 4-6. Effects of solvent types on gelation time of monolithic silica aerogel........................78

Table 4-7. Effects of target density on textural properties of monolithic silica aerogels........82

Table 4-8. List of skeletal density and porosity of monolithic silica aerogels produced using the standard process. The uncertainties are $0.06 \%$ and $0.25 \%$ in skeletal density and porosity respectively.

Table 4-9. Effects of solvent types in gelation on densities and porosities of monolithic silica aerogels. 92

Table 4-10. Effects of solvent types on textural properties of monolithic silica aerogels.......94 
Table 4-11. Effects of pre-hydrolysis catalyst on densities and porosities of monolithic silica aerogels.

Table 4-12. Effects of pre-hydrolysis catalyst on textural properties of monolithic silica aerogels.

Table 4-13. Effects of aging treatment for hydrophobicity on densities and porosities of monolithic silica aerogels 100

Table 4-14. Effects of hydrophobicity on textural properties of monolithic silica aerogels. 100

Table 5-1. List of reagents used for yttria aerogel production. 104

Table 5-2. Resulting gelation time and corresponding densities of the monolithic yttria aerogels. 107

Table 5-3. Effects of target density on textural properties of monolithic yttria aerogels.... 110

Table 5-4. List of reagents used for the organic aerogel processing... 112

Table 5-5. Resulting gelation time and corresponding densities of the monolithic organic aerogels.

Table 5-6. Effects of target density on textural properties of monolithic organic aerogels.119

Table 6-1. List of wire set-implanted silica aerogels prepared with the wire sets for the 30 measurement for thermal conductivity

Table 6-2. List of wire set-implanted organic aerogels prepared with the wire sets for the $3 \omega$ measurement of thermal conductivity. 130

Table 6-3. Physical properties and measurement results of $3 \bowtie$ for monolithic aerogels with implanted wire sets. 


\section{Chapter 1}

\section{Aerogel}

\subsection{Introduction}

Aerogels are ultra-low density and highly porous solids, made by the formation of a wet gel and subsequent solvent removal from the gel by supercritical drying or other methods without collapsing the inherent structure of the original gel. Aerogels primarily result from a two-step preparation method, first a sol-gel step and second a drying step performed under special conditions, i.e., supercritical conditions of evacuation of all liquid phases. There can be an additional step, called the aging step, between the two main steps. The aging of synthesized wet gel from the sol-gel process is oftentimes employed to consolidate the wet-chemical structure by continuous condensation, esterification, dissolution, reprecipitation, depolymerization, and repolymerization processes. Aging is a function of time, temperature, $\mathrm{pH}$, and the nature of the aging fluid.

\subsubsection{Solution-sol-gel process}

The sol-gel process (see Error! Reference source not found..), also known as chemical solution deposition, is a wet-chemistry technique widely used in the fields of material science and ceramic engineering. Sol-gel methods are used primarily for the fabrication of materials (typically metal oxides) starting from a chemical solution which acts as the precursor to an integrated network of either discrete particles or network polymers, the so-called gel. Typical 


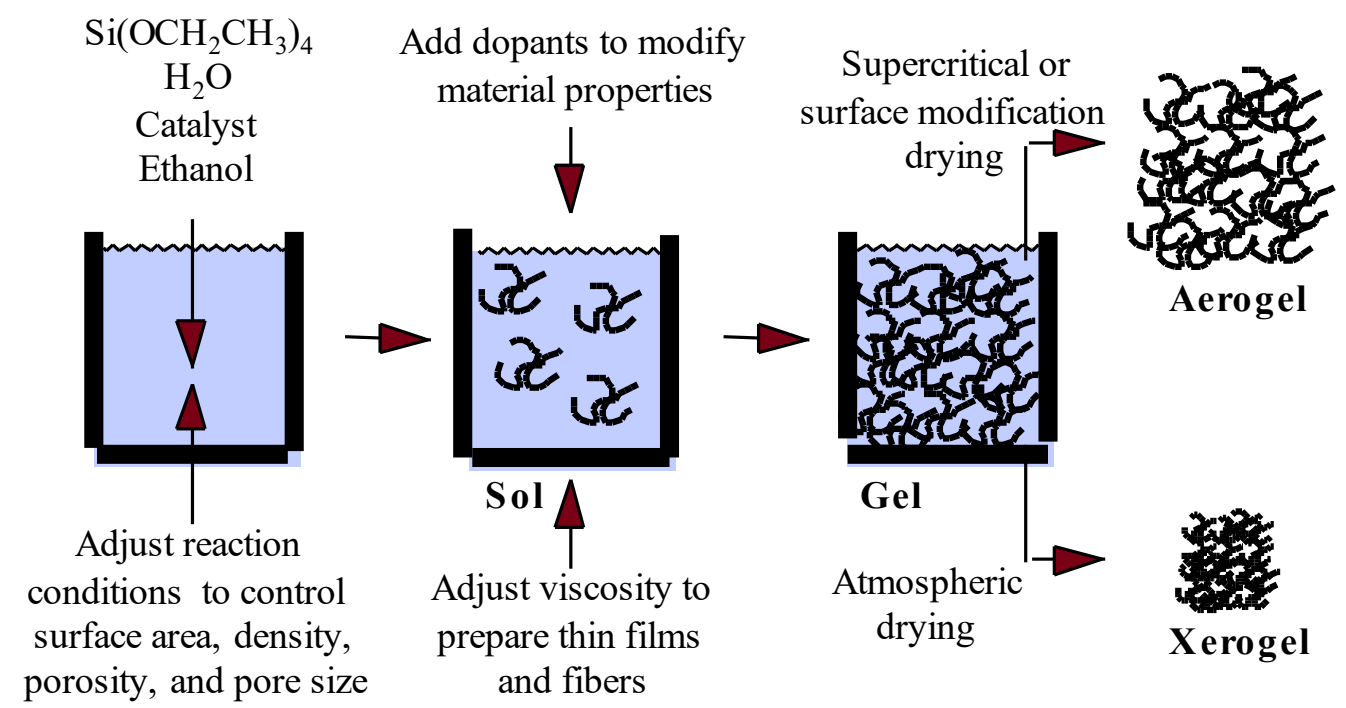

Figure 1-1. Illustration of a standard sol-gel process.

precursors are metal alkoxides and metal chlorides, which undergo various forms of hydrolysis (formation of hydroxyl group hydroxylation) and condensation (formation of bridging bonds oxolation and olation) of appropriate precursor molecules [1]. The sol evolves towards the formation of a gel-like diphasic system containing both a liquid phase and a solid phase whose morphologies range from discrete particles to continuous polymer networks (gel). The typical sol-gel reaction, which involves the use of metal alkoxides, is usually written as follows:

Hydrolysis : $\mathrm{M}(\mathrm{OR})_{\mathrm{n}}+\mathrm{nH}_{2} \mathrm{O} \rightarrow \mathrm{M}(\mathrm{OH})_{\mathrm{n}}+\mathrm{nROH}$

Poly condensation : $\mathrm{M}(\mathrm{OR})_{\mathrm{n}}+\mathrm{M}(\mathrm{OH})_{\mathrm{n}} \rightarrow \mathrm{M}-\mathrm{O}-\mathrm{M}+\mathrm{nROH}$,

$$
\mathrm{M}(\mathrm{OH})_{\mathrm{n}}+\mathrm{M}(\mathrm{OH})_{\mathrm{n}} \rightarrow \mathrm{M}-\mathrm{O}-\mathrm{M}+\mathrm{H}_{2} \mathrm{O}
$$

where $\mathrm{M}$ is metal or $\mathrm{Si}$, and $\mathrm{R}$ is an alkyl group. By adjusting the synthetic conditions, such as the $\mathrm{pH}$, water/precursor ratio, solvent/precursor ratio, and temperature of the reaction system, 
the resulting product can range widely from colloidal solutions, colloidal gels, polymeric gels, or simply precipitates.

There are two important sol-gel methods, namely the alkoxide [2] and the colloidal methods $[3,4,5]$. A third sol-gel method, intermediate between the others but suitable only for silicate systems and based on the chemistry of amino-silicate solutions, has been re-proposed by Maliavski et al. [6]. The most common precursor used in the sol-gel processes is a metal alkoxide in which elements are bonded to a hydrocarbon moiety through oxygen. In addition to the two primary methods, the use of metal salts has been re-evaluated and actively investigated for the last decade, and this has contributed to increasing feasibility of obtaining various species of aerogels which are only possible under very limited conditions [7]. The sol-gel method for yttria aerogel in this dissertation is an example of applying this method and a series of monolithic yttria aerogels were obtained successfully.

The intrinsic sol-gel reactivity of metal alkoxides lies mainly in the steric effects of alkoxy ligands and the inductive effects of the alkoxy ligands. During sol-gel processing of silicon alkoxides, condensation can proceed by combinations of alcohol-organosilanol and waterorganosilanol reactions that have undergone different extents of hydrolysis [8]. According to Voronkov et al., the condensation rate of triorganosilanol decreases with an increase in the length or branching of the chain of the alkyl radical as the longer alkyl chains occupy more space and reduce the chance of having the silanols meet and react with each other during the condensation process [9]. For example, trimethoxysilanol $\left(\left(\mathrm{CH}_{3}\right)_{3} \mathrm{Si}-\mathrm{OH}\right)$ has a higher condensation rate than triethoxysilanol $\left(\left(\mathrm{C}_{2} \mathrm{H}_{5}\right)_{3} \mathrm{Si}-\mathrm{OH}\right)$ because ethyl groups in the triethoxysilanol have more carbons that form longer chains. Likewise, when the alkyl groups are replaced by substituents in tetrafunctional alkoxides that are employed in sol-gel processing as the precursor, it is expected that the substituents that increase steric crowding in the sol-togel state will retard condensation. The methoxy groups $\left(\mathrm{OCH}_{3}-\right)$ of methyltrimethoxysilane 
(MTMS) can be examples of the substituents described above as they can be substituents for the methyl groups of tetramethyl orthosilicate (TMOS). Voronkov et al. also reported that the condensation rate is proportional to the number of silanols on the silicon atom, which results in silanol acidity [9]. This result can be explained on the basis of steric, inductive effects.

While such an analytic review on the sol-gel process helps to understand the basic chemistry, the virtual reactions that occur during the sol-gel process are much more complicated than that introduced in Eqs. (1.1) and (1.2). This is because it is nearly impossible to accurately define when the intermediate products appear and how they are involved in the main reactions as well as the molecular structure of the final products. However, it is these intermediate products, whose formation and control are not fully understood which fundamentally influence the sol-gel process and their role in the aerogel production process remains to be clarified. Smallangle X-ray scattering (SAXS) [10, 11], Rayleigh scattering [12], and NMR studies [13] have been actively employed to study the sol-gel reactions at the scale of the nanostructured molecular units and many reports have been produced to provide qualitatively informative information on how and when the $\mathrm{M}(\mathrm{OR}) \mathrm{n}$ is transformed into the $\mathrm{M}(\mathrm{OH}) \mathrm{n}$ followed by the metal or silica oxide in some sol-gel routes. On the other hand, the catalysis that simultaneously proceeds, transforming the target reactants, has yet to be analyzed by the above methods in the aerogel formation process. The catalyst used in the sol-gel process is often involved in unintended reactions such as trans-esterification and synerisis of the final product that result in the structural change in nature of the produced gel. It further interferes with the supercritical extraction process by reacting with liquid or supercritical $\mathrm{CO}_{2}$ or retarding the solvent exchange process.

The precursor sol can either be deposited onto a substrate to form a film (e.g., by dip coating or spin coating), cast into a suitable container with the desired shape (e.g., to obtain monolithic ceramics, glasses, fibers, membranes [14], bulk aerogels), or used to synthesize powders (e.g., 
microspheres, nanospheres). The sol-gel approach is, in general, a cost-effective and lowtemperature technique that allows for fine control of the resulting product's chemical composition. Small quantities of dopants, such as organic dyes and rare earth elements, can also be introduced into the sol and end up uniformly dispersed in the final product. The sol-gel process can be used in ceramic processing and manufacturing for investment casting, or as a means of producing very thin films of metal oxides for various purposes.

\subsubsection{Supercritical drying process}

After gelation, the wet gel may optionally be aged in its mother liquor to strengthen the gel networks, or in another solvent for modification of the inherent gel property after which it is then rinsed by the mother liquor [8]. Then this mother liquor can be removed by the drying process to obtain gels which have different shapes and properties depending upon the drying method. With respect to the drying method applied, a conventionally dried gel is termed a xerogel, a freeze dried one a cryogel, and a supercritically dried one an aerogel [1].

Supercritical drying prevents the formation of a liquid-vapor meniscus during the phase change process of drying, which recedes during the emptying of the pores in the wet gels. This meniscus forms due to the surface tension of the liquid. A capillary pressure $\left(\mathrm{P}_{\mathrm{S}}\right)$ gradient is established on the pore walls, which can be described as,

$$
\begin{gathered}
2 \sigma r \cos \theta=r^{2} h \rho g \\
\rho g h=P_{s}=(2 \sigma / r) \cos \theta
\end{gathered}
$$

where $\theta$ is a contact angle between the solid and the liquid, $\sigma$ is surface tension, $r$ is the pore radius, $h$ is the depth of the pore, $\rho$ is density of the solvent, and $g$ is gravity. For example, when

the pore radius is $20 \mathrm{~nm}$ and alcohol is used as the solvent, $\sigma=22.75 d y n / \mathrm{cm}, \rho=0.7893 \mathrm{~g} / \mathrm{cm}^{3}$, 
and the corresponding capillary force is $22.5 \mathrm{~atm}$. This force is great enough to collapse the majority of the pore volume, resulting in low-density, porous silica with less remarkable properties [15]. To avoid this pressure and the resulting collapse of the gel network, it is necessary to eliminate the liquid surface tension. One convenient way to do so is to transform the liquid into a supercritical fluid with no surface tension. Supercritical extraction of the liquidphase impregnating the porous volume of the wet gel permits the recovery of a kind of "dry

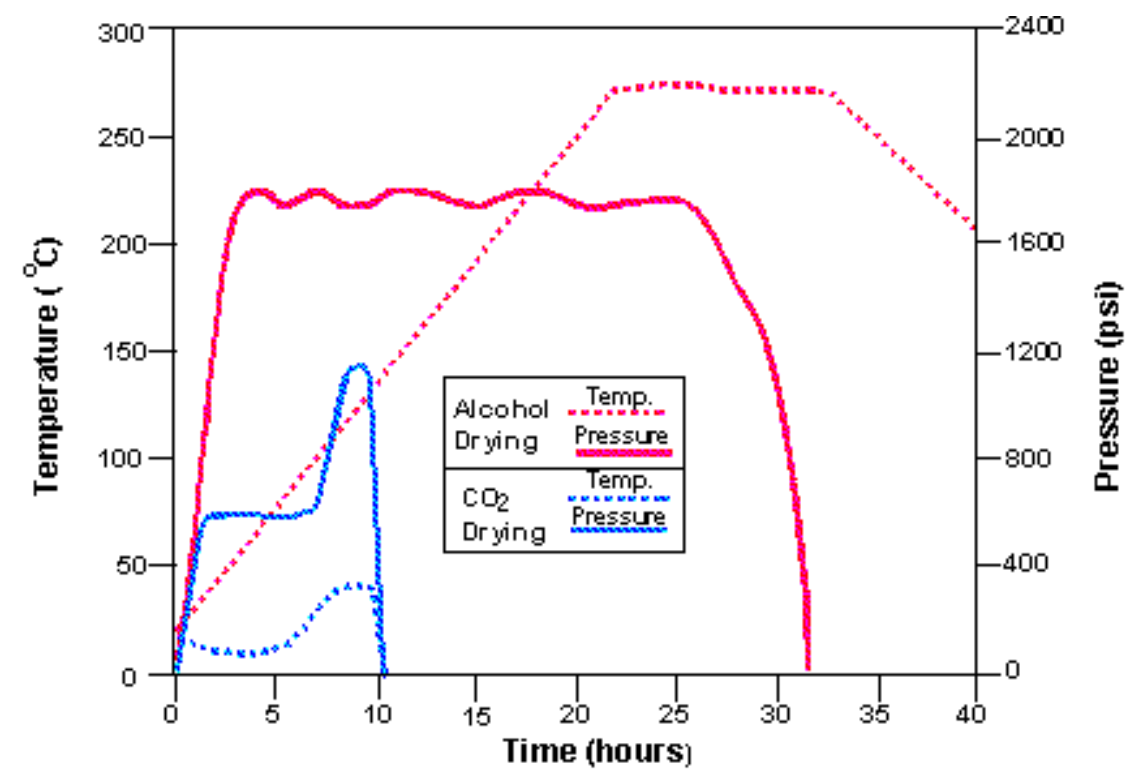

Figure 1-2. Comparison of required pressures and temperatures for supercritical drying ( $\mathrm{CO}_{2}$ vs alcohol) [17].

solid image" of the wet material. This drying procedure yields a porous structure quite intact by avoiding the pore collapse phenomenon [16].

The supercritical drying process can be divided into two categories: the high temperature method and the low temperature method. They both circumvent the capillary stress either by transferring the solvent into the supercritical state or by replacing the solvent with either liquid or supercritical $\mathrm{CO}_{2}$, thus eliminating any liquid-vapor interface inside the sol-gel product 
during solvent extraction. Figure 1-2 shows typical processing pathways in temperature and pressure during the COLD method and the HOT method of supercritical drying process [17].

The high temperature method requires a heat proof autoclave with appropriate safety system to secure the safe and stable drying process. The low temperature method, on the other hand, requires less stringent system requirements for safety. This advantage has made the low temperature method more popular in the aerogel industry. Table 1-1 lists critical constants (critical temperature, $T_{c}$, and critical pressure, $P_{c}$ ) of a few solvents which are commonly used for, or related to, the supercritical extraction process.

Table 1-1. Critical constants of fluids used in supercritical extraction.

\begin{tabular}{|c|c|c|c|c|}
\hline \multirow[b]{2}{*}{ Name } & \multirow{2}{*}{ Formula } & \multirow{2}{*}{$\begin{array}{c}\text { Critical } \\
\text { temperature } \\
(\stackrel{\circ}{ } \mathrm{C})\end{array}$} & \multicolumn{2}{|c|}{ Critical pressure } \\
\hline & & & (psi) & (Mpa) \\
\hline Carbon dioxide & $\mathrm{CO}_{2}$ & 31.1 & 1073 & 7.36 \\
\hline Nitrous oxide & $\mathrm{N}_{2} \mathrm{O}$ & 36.5 & 1054 & 7.24 \\
\hline Freon 116 & $\mathrm{CF}_{3} \mathrm{CF}_{3}$ & 19.7 & 432 & 2.97 \\
\hline Methanol & $\mathrm{CH}_{3} \mathrm{OH}$ & 240 & 1155 & 7.93 \\
\hline Ethanol & $\mathrm{C}_{2} \mathrm{H}_{5} \mathrm{OH}$ & 243 & 927 & 6.36 \\
\hline Water & $\mathrm{H}_{2} \mathrm{O}$ & 374 & 3204 & 22.0 \\
\hline
\end{tabular}

Presently, high temperature supercritical drying in alcohol is referred to as the HOT method for aerogel production and low temperature supercritical drying in $\mathrm{CO}_{2}$ is called the COLD 
method. The resulting materials from the HOT method are generally hydrophobic since their surfaces are covered by alkoxy groups. The COLD method does not favor such processes, and as a consequence, it leads to more hydrophilic solids.

In the COLD method, a crucial stage in silica aerogel production in the COLD method is alcohol diffusion through alcogel (the alcohol filled gel) to $\mathrm{CO}_{2}$ as ethanol and methanol are dominantly used as the solvent in the aerogel production process. Mukhopadhyay et al. proposed a mechanistic supercritical drying model for producing crack-free aerogels, and elucidated the requirement of such a process by focusing on mass transfer modeling through a silica aerogel assuming parallel cylindrical mesopores with a known pore size distribution [18]. Their results lead to the conclusion that most of the ethanol is removed by spillage as a result of supercritical $\mathrm{CO}_{2}$ dissolution in the pore liquid, rather than by convective evaporation. Higher pressures, lower temperatures, a lower supercritical $\mathrm{CO}_{2}$ diffusion rate and thicker gels all slow down the supercritical drying process and are conducive to producing crack-free transparent aerogels.

Rogacki et al. and Novak et al. investigated the kinetics of the second stage by identification of the binary coefficients of ethanol-liquid $\mathrm{CO}_{2}[19]$ and methanol-liquid $\mathrm{CO}_{2}$ [20], respectively. The analysis was simplified to the superposition of diffusion in two infinite plates:

$$
C\left(F_{0}, x, y\right)=f\left(F_{0}, x\right) f\left(F_{0}, y\right)
$$

where $C$ is the alcohol concentration, $f$ is the solution for an infinite flat plate, $x$ and $y$ are the coordinates, and $F_{0}$ is the Fourier number, defined as

$$
F_{0}=\frac{D_{A B} t}{L^{2}}
$$

where $D_{A B}$ is the diffusion coefficient of ethanol- $\mathrm{CO}_{2}$ in the gel sample, $t$ is the time of the diffusion process, and $L$ is the length of the shorter sample edge. In the morphological study, the concentration of methanol on the interface between the transparent and non-transparent areas 
was estimated to be $5 \%$ by using the van de Waals fluid equation [21]. This $5 \%$ interface seen as a projection on the sample face satisfies the following condition:

$$
C_{5 \%}=f\left(F_{0}, x\right) f\left(F_{0}, L / 2\right)
$$

Thus, the width of the damaged zone, $x_{5 \%}$, where cracks or collapses of the gel structure occur during the depressurization step due to the evaporation of unextracted methanol, can be determined from Eq. (1.8):

$$
x_{5 \%}=f^{-1}\left(F_{0}, \frac{C_{5 \%}}{f\left(F_{0}, L / 2\right.}\right)
$$

Since the values of $t$ and $L$ are known from the actual experiment, Eq. (1.8) makes it possible to determine the value of $D_{A B}$. Minimization of the damaged zone is a primary condition when transparent silica aerogel is produced as it directly affects the transparency of the product.

\subsubsection{Ambient drying process}

A promising technique which can be applied on a large scale for industrial purposes is known as "ambient-pressure drying". This method relies on passivation of the pore surface, inside the gel, so as to impede further formation of new siloxane bonds by condensation reactions when the gel network is compressed under the drying stresses. Such a passivation can be induced by silylation, for example, with trimethylchlorosilane. At the end of the solvent evaporation process, the passivated aerogel monolith no longer experiences capillary stresses, and thus it can resume its wet size by a "spring-back" effect. More recently, a new technique has been applied to hydrogels made from sodium silicate (water glass) in hexamethyldisiloxane (HMDS) solvent. This new technique is considered as one of the promising candidates for costeffective processing [16]. 


\subsection{Historical Background}

Aerogels were first developed by Samuel S. Kistler in 1931 [22]. After proving experimentally that the more familiar liquid-based gels or jellies were an open solid network of cells permeated by liquid, he was able to make the first silica aerogels with densities ranging from $0.02 \mathrm{~g} / \mathrm{cm}^{3}$ to $0.1 \mathrm{~g} / \mathrm{cm}^{3}$ [22]. In this process, Kistler prepared a wet gel by using sodium silicate as a precursor. He then soaked the synthesized gel in alcohol for the solvent substitution because the gel was originally water-based. After the solvent substitution, Kistler heated the alcohol and gel in a closed container to a high temperature and pressure (80 atmospheres and $240^{\circ} \mathrm{C}$ ) and slowly depressurized the vessel. This allowed the alcohol, now a vapor, to escape, leaving an air-filled cellular matrix, which he coined an aerogel [23].

Rather than commercialize such an interesting invention, Kistler completed a license agreement with the Monsanto Corp. for the production of silica aerogel in the early 1940's. Monsanto began production in a plant in Everett, Massachusetts, and sold products for many years under the trade name "Santocel" with images shown in Figure 1-3 and Figure 1-4 [24].

The first aerogel products were sold as insulation filler for an oxygen-producing plant and a national line of freezers, and as thickening agents for inks used in cigarette packages, screwworm salves for sheep, and in the jelly of fiery Napalm bomb for the US military. Santocel also became an essential ingredient in the manufacture of silicone rubber. The product, however, 


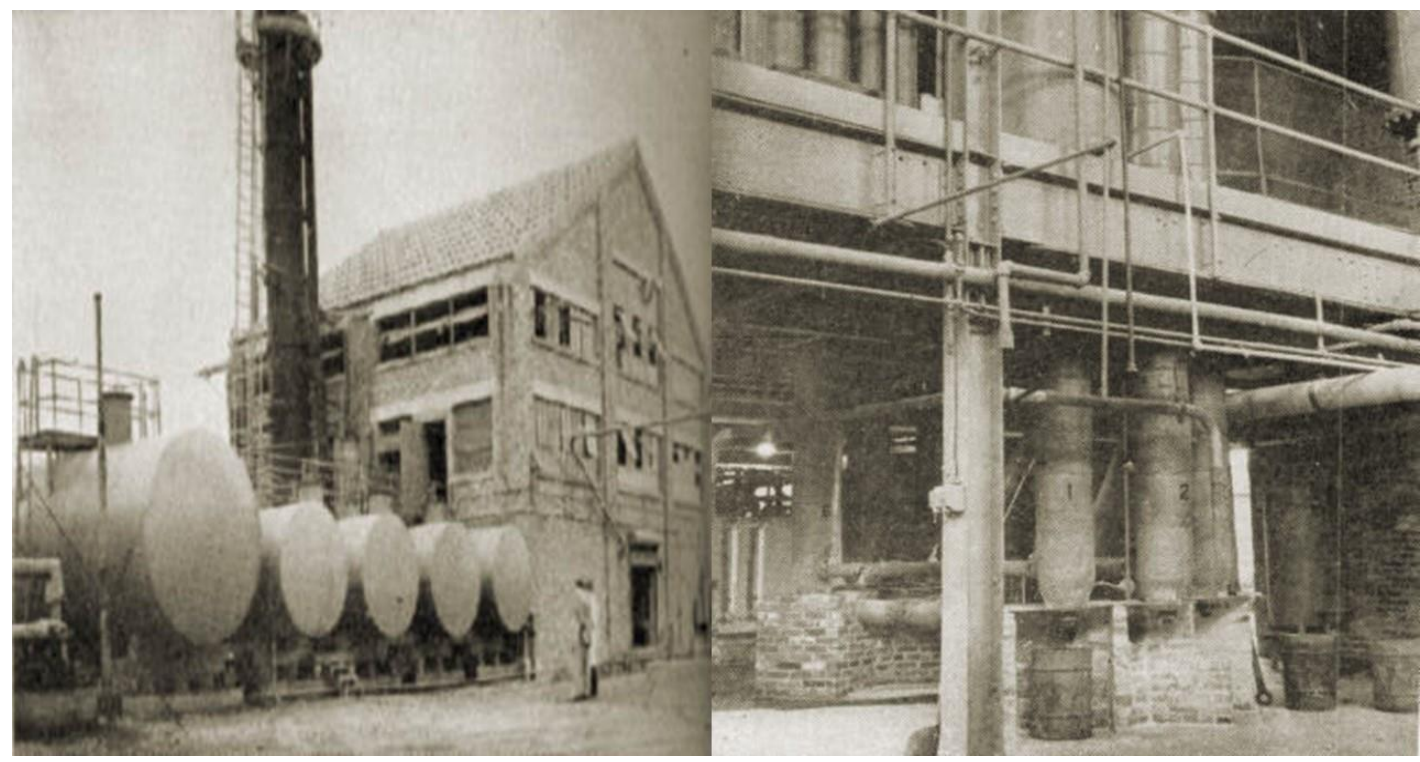

Figure 1-3. First large-scale production unit for silica aerogel in Boston. Alcohol storage tanks in foreground (left). Three of the autoclaves used to dry the gels (right) [24].
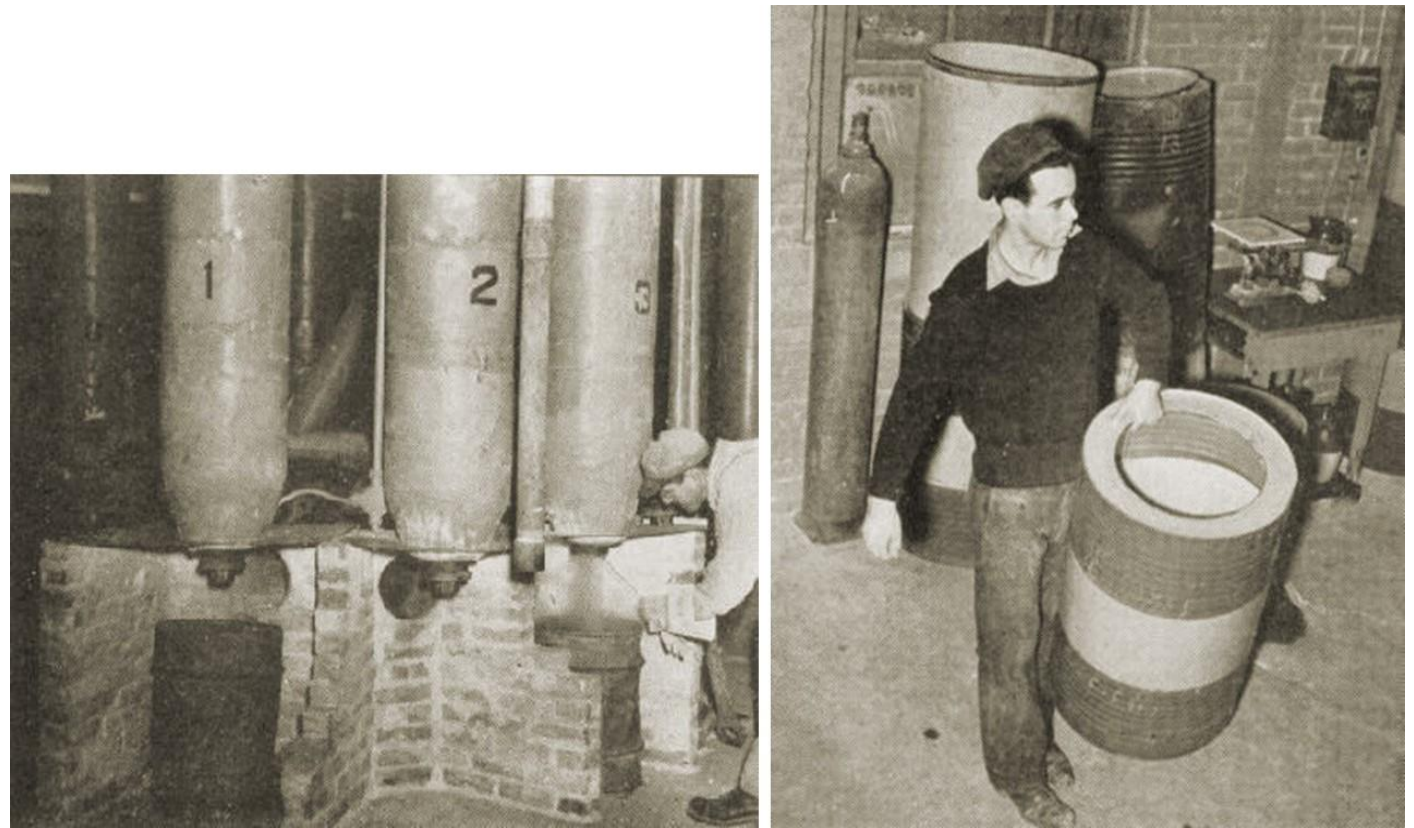

Figure 1-4. The produced aerogel is removed from the autoclave (left) and moved in a container (right). The first aerogel product was a mixture of randomly shaped chunks and powders and it was crashed into powders with relatively uniform size. The final product weighed only 3-5 pounds per cubic foot [24]. 
was discontinued from production in the 1960's due to its high manufacturing cost and insufficient knowledge of the outstanding properties of their own product which creates a competitive advantage relative to others in the industry [24].

Despite much interest in the properties of aerogels, it was not until the 1980's that significant research and studies of aerogels markedly increased. In 1975, Teichner began employing alkoxide as a chemical precursor instead of sodium silicate in the production process [25]. Silica alkoxides, such as tetraethyl orthosilicate (TEOS) and tetramethyl orthossilicate (TMOS), are alcohol-based precursors which enable the wet gel synthesis to proceed without the solvent substitution step. They also show an acceptable reaction rate in the hydrolysis and poly condensation processes as compared to that of sodium silicate. These advantages with the silica alkoxide enabled simplification of the chemistry for the wet gel preparation and offered the ability to tune the gel structure. Ten years later, Hunt found that the alcohol traditionally used in the supercritical drying process can be replaced with liquid carbon dioxide (the COLD method previously described) as it has great miscibility without chemical reaction [26]. This advancement meant he also could reduce the process time from 2-3 days to $8-10$ hours by using liquid $\mathrm{CO}_{2}$ with considerably less expensive equipment.

In the 1990's, researchers started broadly using drying control chemical additives (DCCAs) in order to pursue the drying process under ambient conditions [8]. This later enabled the spin coating method to produce thin film aerogel for various coating applications $[27,28,29,30,31$, $32,33]$. These advances in the aerogel production process resulted in marked increases in the development of various aerogel species. They even triggered early commercialization of aerogel products in the thermal insulation industry [34].

There has been more diversification in investigation and refining of the aerogel process since 2000. A new rapid supercritical extraction (RSCE) method was experimented with, in which the sol-gel precursors were themselves brought to a supercritical state inside a pressurized 
mold [35]. The ambient pressure drying method was extended to aerogels produced from sodium silicate [36]. The use of metal salt as a precursor, thanks to the proton scavenger of the epoxide, was further extended to a range of materials comprising $\mathrm{SnO}_{2}$ [37], $\mathrm{Y}_{2} \mathrm{O}_{3}$-stabilized $\mathrm{ZrO}_{2}$ aerogels [38], aerogel thermites with dispersed $\mathrm{Al}$ nanoparticles [39], $\mathrm{SiO}_{2}$ aerogels with dispersed metal nanoparticles [40] or with CdS nanoparticles grown by lithography inside the aerogel [41], and chitosan $-\mathrm{SiO}_{2}$ hybrid aerogels with dispersed gold particles [42]. In addition, the development of organic aerogels including cellulose aerogel [43], the combination of resorcinol/phloroglucinol-formaldehyde/furfuryl alcohol, and the carbon aerogels derived from them became organic compounds for hybrid organic-inorganic aerogels [44, 45].

Aerogels are now commercialized by several companies, among them Cabot Corp. which produces aerogel particulate insulators for windows [46], Nano HiTech [47] and Okagel also produce insulating products [48], American Aerogel Corp. produces open-cell foam materials [49], Birdair Company produces membranes with aerogel inserts [50], and Aspen Aerogels Inc. produces flexible insulation products [51].

\subsection{Definition}

Aerogel has been defined from two different standpoints since the 1990's, when the ambient drying process was proposed as an alternative method to the supercritical drying process. The first definition is based on the processing method, which consequently determines the nature of the resulting aerogel. This has been upheld as the sole definition for over five decades, ever since the first aerogel was produced. The second definition is based on the key properties of aerogel. As mentioned above, it was found that the xerogel production process can produce materials with compatible characteristics to those of supercritically dried aerogels by modifying 
the chemical preparation technique. For example, silica aerogels produced from the ambient drying method can have surface areas as large as approximately $60 \%$ compared to that of silica aerogel produced from the conventional aerogel process [52]. Figure 1-5 illustrates typical pore size distributions of silica aerogels measured by the nitrogen adsorption-desorption and calculated by applying the Barrett-Joyner-Halenda method (BJH) to the desorption branch of the isotherm [53]. From this figure, one can clearly see the fundamental differences in structural properties of aerogels prepared from the ambient drying technique compared with the supercritical drying technique.

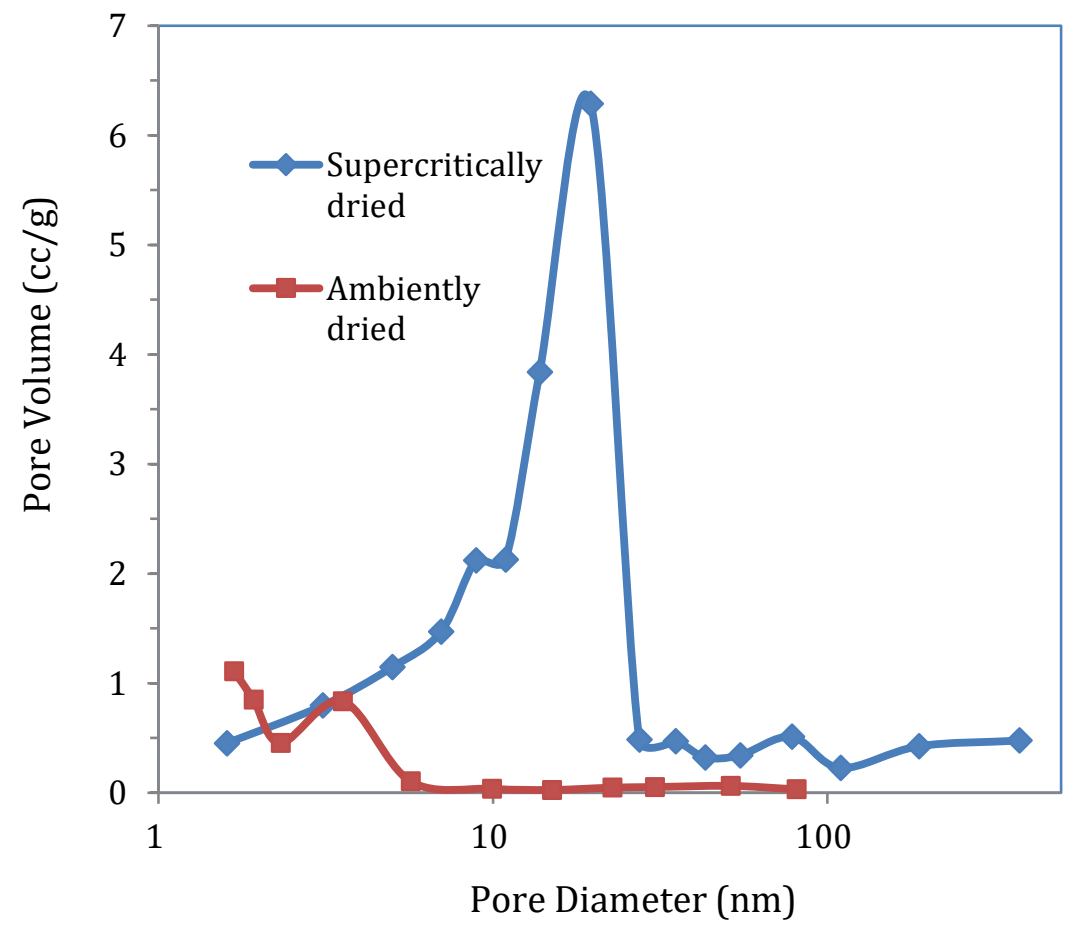

Figure 1-5. Examples of pore size distributions of ambiently dried silica aerogel (square, [53]) and supercritically dried silica aerogel (diamond, Aerogel Research Lab at University of Virginia). 
While both definitions have been accepted for nearly 30 years, the conventional aerogel process has been optimized and modified for various applications. As a result, the surface area of aerogels from some optimized methods has increased by over $30 \%$.

Typical silica aerogel consists of approximately $95 \%$ air, while the remaining $5 \%$ is a thin, wispy matrix of silica. The unique microstructure (Figure 1-6) leads to many extraordinary properties [54]. Aerogels have the lowest density, lowest thermal conductivity, lowest dielectric constant, lowest acoustic velocity, and lowest refractive index of any known solid. The thermal conductivity of aerogels can be two to three orders of magnitude smaller than that of bulk silica glass and values as low as $0.003 \mathrm{~W} / \mathrm{m} \mathrm{K}$ have been recorded [55].

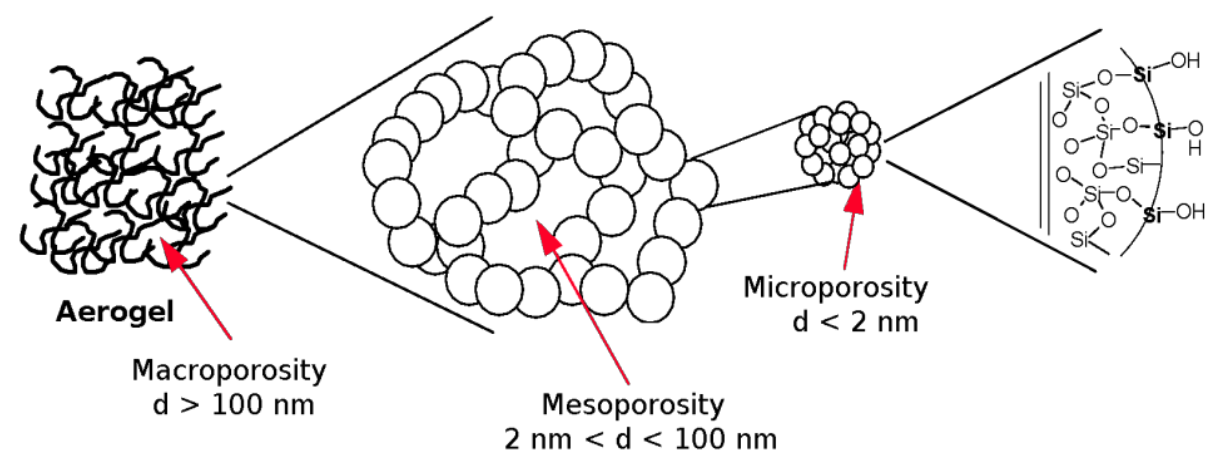

Figure 1-6. Complex aerogel pore structure describing macro-, meso-, and micro-porosity in addition to surface silanol groups of a "standard" un-modified silica aerogel [54].

Due to its extraordinarily low thermal conductivity, aerogels are well-known thermal insulating materials. It has been considered as an insulating solution in many areas as a replacement to conventional materials due to the global environmental issues related to energy efficiency and preservation of natural resources. In recent studies, more attention has been paid to aerogel coating technology for applications in the MEMS field since Yokokawa et al. reported 
the fabrication of thin film aerogel [56]. The Aerogel Research Lab at UVA (AeRL) found that a silicon wafer can be coated by a silica aerogel layer via dip coating, drop coating, and spin coating methods [57].

\subsection{Species and Applications}

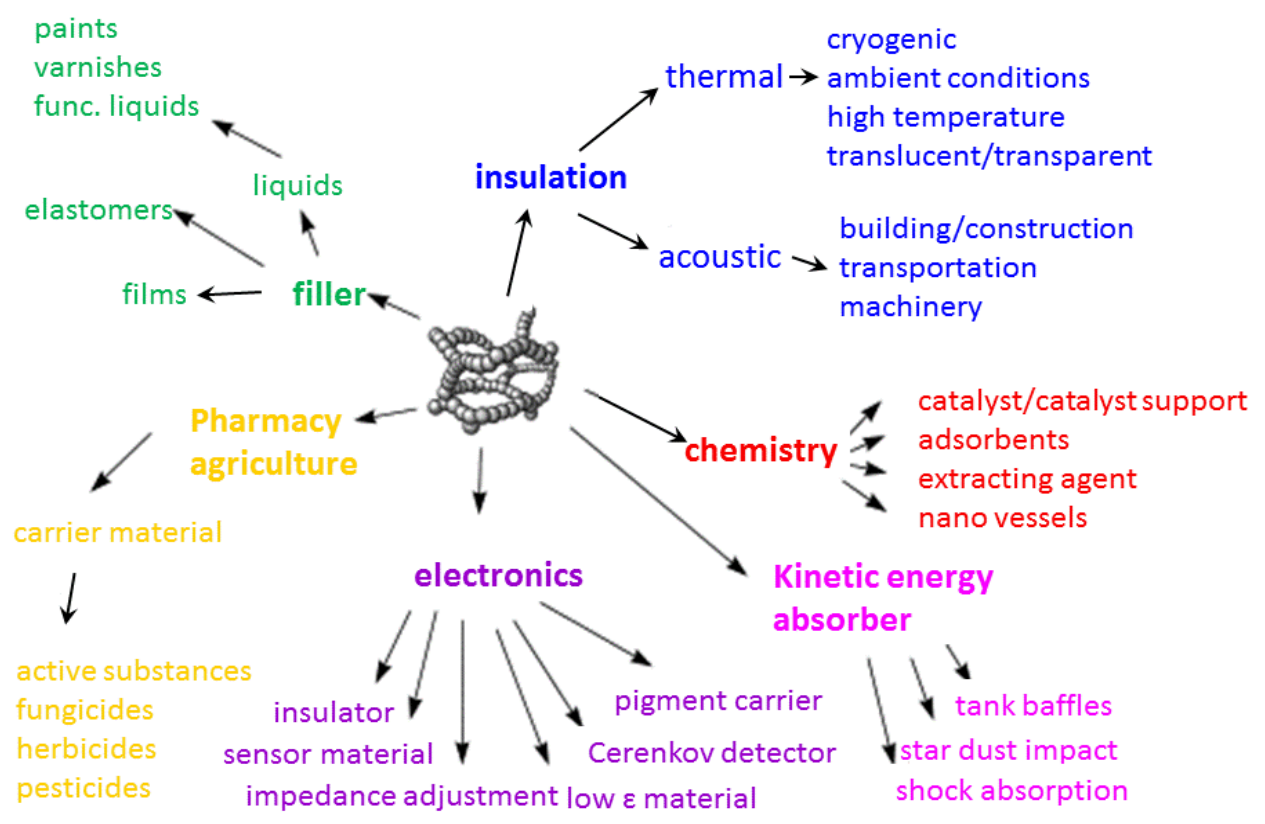

Figure 1-7. Application fields of aerogel products [58].

As introduced in the history and the definition, aerogel can virtually exist in almost all kinds of solids on the earth thanks to its synthetic production method. Aerogels are categorized into two main species: organic and inorganic. Representative inorganic aerogels are silica, 
titania, alumina, niobia, and zirconia. With the exception of silica aerogel, the others are also called metal oxide aerogels. They are commonly prepared by using a metal alkoxide as a precursor and recent studies have demonstrated an alternative method by using a metal salt that replaces the alkoxide. The applications for such metal oxide aerogels are as various as catalysis, dielectric shield, and insulation for buildings, plants, and aircrafts.

Carbon aerogel receives great attention among organic aerogels because of its extraordinary properties in the electronics field. In addition, organic-inorganic hybrid aerogels are expected to be a new solution with applications in some advanced technology areas such as thermal protection system (TPS) for space vehicles and super capacitors in the fuel cell industry. Figure 1-7 shows the application fields for aerogel products [58], yet they are evolving rapidly as many industrial trends have changed over time.

In Chapter 2, thermal conductivity of aerogel will be discussed by reviewing reported references considering amorphousness and high porosity of the aerogel's structure. Then it will introduce research objectives of this dissertation followed by outlines of the next chapters. 


\section{Chapter 2}

\section{Thermal Conductivity of Aerogel}

This chapter describes methods for estimating the thermal conductivity of aerogel. The first section describes thermal conduction in amorphous materials by considering thermal transport by vibrations (Section 2.1). The method for defining porosity of aerogel is described in Section 2.2, as aerogels are well known to be amorphous and porous materials. The next two sections then introduce the effective medium theory, a method for considering thermal transport in heterogeneous materials macroscopically (Section 2.3) and the modification necessary when considering nanoscale thermal transport in porous materials (Section 2.4). In Section 2.5, the sensitivity of the thermal conductivity of aerogels to the porosity is discussed. Section 2.6 describes the necessity of fine adjustment of the thermal conductivity of aerogel in the thermal insulation industry and introduces the research objectives of the dissertation. Finally, a brief outline of the following chapters is provided in Section 2.7 .

\subsection{Thermal Conductivity of Amorphous}

\section{Materials}

The thermal conductivity of amorphous materials is known to be reduced compared to crystalline materials. Unlike the lattice vibrations of crystalline materials, those of amorphous materials are not best described by waves, but rather by damped local oscillations [59]. In perfect 
crystalline solids, the lattice vibrations can be described as traveling waves. All amorphous solids, however, have additional, low-energy excitations dominating the specific heat below a few Kelvin. Below $1 K$ the specific heat approaches a linear variation with temperature. The excitations associated with this specific heat are believed to lead to the characteristic thermal conductivity varying as the square of the temperature [60].

Another universal feature of amorphous solids is that at high temperatures their thermal conductivity increases with increasing temperature and approaches a nearly temperatureindependent value near the softening temperature [60]. In a 1989 paper, Cahill and Pohl used a modified version of Einstein's theory of lattice vibrations to calculate an expression for the thermal conductivity of glasses $\left(k_{C P}\right)$ [61]. They obtained a thermal conductivity given by,

$$
k_{C P}=\left(\frac{\pi}{6}\right)^{1 / 3} k_{B}\left(\frac{N_{A} \rho}{M}\right)^{2 / 3} \times \sum_{i} v_{i} \frac{T^{2}}{\theta_{i}^{2}} \int_{0}^{\theta_{i} / T} \frac{x^{3} e^{x}}{\left(e^{x}-1\right)^{2}} d x
$$

where $N_{\mathrm{A}}$ is Avogadro's number, $\rho$ is the density, and $M$ is the molecular weight. The sum over $i$ includes the two transverse modes and one longitudinal sound mode, and $v_{i}$ is the mode velocity. $k_{\mathrm{B}} \theta_{\mathrm{i}} / \hbar$ is the cutoff frequency for mode $i$ with,

$$
\theta_{i}=\frac{\hbar}{k_{B}}\left(\frac{6 \pi^{2} N_{A} \rho}{M}\right)^{1 / 3}
$$

Cahill and Pohl found this formula to be in reasonable agreement with data for amorphous silica for temperatures above $50 \mathrm{~K}\left(-223^{\circ} \mathrm{C}\right)[61]$.

Hopkins et al. presented methods for estimating the thermal conductivity of amorphous materials [62]. The three major phonon scattering events in crystalline-structured materials are due to impurities, boundaries, and Umklapp processes $\left(\propto T^{-l}\right)$. On the other hand, the temperature dependency in thermal conductivity of amorphous materials follow that of the heat 
capacity and this leads to the following definition of a minimum vibrational scattering length scale (akin to a minimum "mean free path" $\lambda_{\min }$ ), assuming a minimum thermal conductivity given by,

$$
\lambda_{\min }=\mathrm{n}^{-1 / 3}
$$

where $n$ is a number density [62]. Kaviany stated that the range of the mean-free paths differs greatly among crystalline, polycrystalline, and amorphous solids, and the shortest is reported to be for phonons in amorphous silica [63]. For amorphous silica, this length scale is $0.6 \mathrm{~nm}$, and it is nearly the same as the average lattice constant $(0.4 \mathrm{~nm})$ due to lack of crystallinity on the order of the atomic spacing [63].

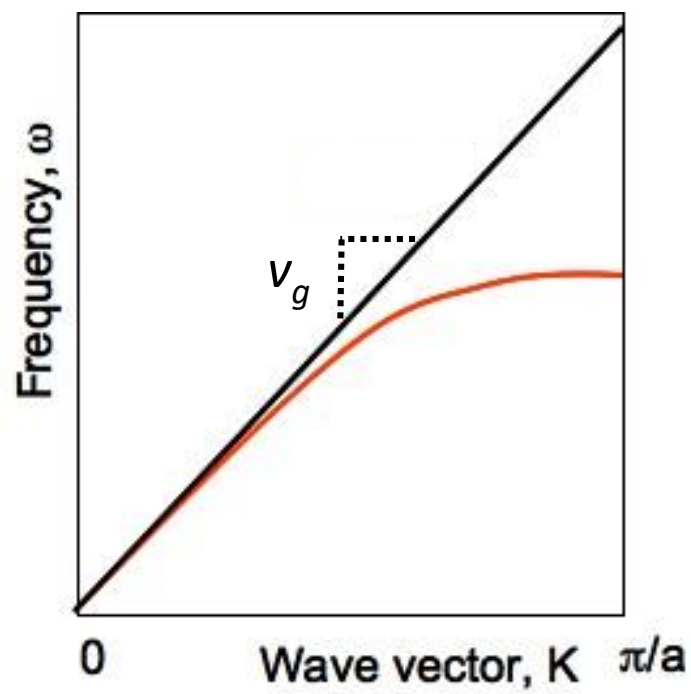

Figure 2-1. Debye approximation to the dispersion (black line) and definition of group velocity. Where $a$ is the interatomic spacing.

As these values are fitted with the Debye model, the group velocity $\left(v_{g}\right)$ and the phase velocity $\left(v_{p}\right)$ of phonons were introduced as shown in Figure 2-1 by considering the Debye approximation, 


$$
v_{g}=\frac{\Delta \omega}{\Delta \bar{k}}, \quad v_{p}=\frac{\omega}{\bar{k}}, \quad v_{g}=v_{p}
$$

In amorphous solids, however, these two velocities are unequal. Hopkins et al. defined the phase velocity as,

$$
v_{p}=\frac{\lambda_{\min }}{\tau_{\min }}=\frac{n^{-\frac{1}{3}}}{\pi / \omega}=\frac{\omega}{\pi n^{\frac{1}{3}}}, \quad \tau_{\min }=\frac{\pi}{\omega}[62] .
$$

He then applied this definition to the density of states (DOS) per unit frequency,

$$
D O S=\frac{1}{2 \pi^{2}} \bar{k}^{2} d \bar{k}=\frac{1}{2 \pi^{2}} \frac{\omega^{2}}{v_{p}^{2}} \frac{d \omega}{v_{g}}=\frac{n^{2 / 3}}{2} \frac{d \omega}{v_{g}}=\frac{n^{2 / 3}}{2} \frac{1}{v_{g}}[62]
$$

By substituting the result for the DOS into the expression for heat capacity, one obtains,

$$
\begin{gathered}
C=\sum_{i} \int_{0}^{\omega_{c, j}} \hbar \omega(D O S) \frac{d f}{d T} d \omega=\sum_{i} \int_{0}^{\omega_{c, i}} \hbar \omega \frac{n^{2 / 3}}{2 v_{g}} \frac{e^{\frac{\hbar \omega}{k_{B} T}}}{\left(e^{\frac{\hbar \omega}{k_{b} T}}-1\right)^{2}} \frac{\hbar \omega}{k_{B} T^{2}} d \omega \\
=\frac{\hbar^{2}{ }^{2} / 3}{2 \mathrm{k}_{\mathrm{B}} \mathrm{T}^{2}} \sum_{\mathrm{i}} \frac{1}{\mathrm{v}_{\mathrm{g}, \mathrm{i}}} \int_{0}^{\omega_{c, \mathrm{i}}} \omega^{2} \frac{\mathrm{e}^{\frac{\hbar \omega}{\mathrm{k}_{\mathrm{B}}}}}{\left(\mathrm{e}^{\frac{\hbar}{\mathrm{k}_{\mathrm{b}} \mathrm{T}}}-1\right)^{2}} \mathrm{~d} \omega .
\end{gathered}
$$

Let $x=\hbar \omega / k_{B} T$, then,

$$
C=\frac{n^{2} / 3 k_{B}^{2}}{2 \hbar} T \sum_{i} \frac{1}{v_{g, i}} \int_{0}^{\frac{\theta_{D}}{T}} \omega^{2} \frac{e^{x}}{\left(e^{x x}-1\right)^{2}} d x
$$

From the above equation, it is demonstrated that the heat capacity $(C)$ is proportional to the temperature ( $T$ ) when $T$ is much less than the Debye temperature $\left(\theta_{D}\right)[64]$. 
Finally, Hopkins et al. introduced the newly modified thermal conductivity model for amorphous solids by applying Eq. (2.6), along with the phase velocity term in Eq. (2.1) as

$$
\begin{gathered}
k_{\min }=\frac{1}{3} \sum_{i} \int_{0}^{\omega_{c}} \hbar \omega(D O S) \frac{d f}{d T} v_{g} \lambda_{\min } d \omega, \quad \lambda_{\min }=v_{p} \cdot \tau_{\min } \\
k_{\min }=\frac{\hbar^{2} n^{1 / 3}}{6 k_{B} T^{2}} \sum_{i} \int_{0}^{\omega_{c, i}} \omega^{2} \frac{e^{\frac{\hbar \omega}{k_{B} T}}}{\left(e^{\frac{\hbar \omega}{k^{T}}}-1\right)^{2}} d \omega[65] .
\end{gathered}
$$

This is the minimum thermal conductivity model presented previously for disordered, layered solids [65]. Later, it has been used for predictions in amorphous solids by modification with the expression of the Debye temperature for each polarization $j\left(\Theta_{\mathrm{j}}\right)$,

$$
\begin{gathered}
\theta_{j}=\frac{v_{g, j} \hbar\left(6 \pi^{2} n\right)^{\frac{1}{3}}}{k_{B}} \\
k_{\text {min,MOD }}=\left(\frac{\pi^{2}}{36}\right)^{1 / 3} n^{2 / 3} k_{B} \sum_{j} \frac{T}{\theta_{j}} v_{g, j} \int_{0}^{\theta_{j} / T} x^{2} \frac{\exp (x)}{(\exp (x)-1)^{2}} d x[62] .
\end{gathered}
$$

Equation (2.9) was presented without deviation previously [65]. This equation describes the heat propagation through the solid at a group velocity $\left(v_{g}\right)$ which is different than the phase velocity $\left(v_{p}\right)$ limited by the atomic oscillations [62].

\subsection{Porosity of Aerogel}

Based on what has been described, it is possible to calculate the thermal conductivity of an aerogel assuming an entirely amorphous-structured material. However, unlike most typical amorphous materials, aerogels are extremely porous materials and this factor has to be taken 
into account as the thermal conductivity is estimated. To have it considered, we utilize the porosity factor calculated by the following formula [66]:

$$
\% \text { of porosity for aerogel }=\left(1-\frac{\rho_{\mathrm{b}}}{\rho_{\mathrm{s}}}\right) \times 100
$$

where $\rho_{b}$ is the apparent (bulk) density and $\rho_{s}$ is the skeletal density of the porous material. The bulk density of aerogel is easily calculated from the measured mass and volume of the aerogel monolith. Since it is an intrinsic variable of a material, the $\rho_{b}$ of the bulk aerogel is the same as that of the monolithic aerogel unless any of the three dimensions of the aerogel is less than the aerogel's pore size $(\sim 20 \mathrm{~nm}$ on average $)$.

$$
\rho_{b}: \text { bulk density }
$$

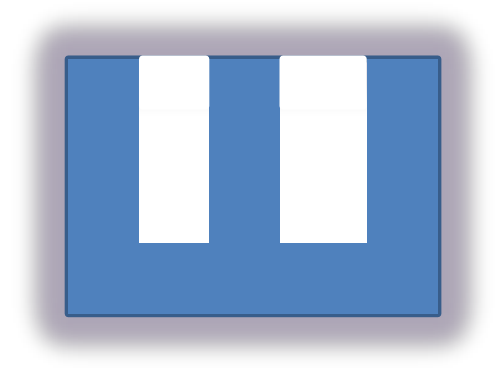

$\rho_{s}:$ skeletal density

porosity $\propto\left(\rho_{s}-\rho_{b}\right)$

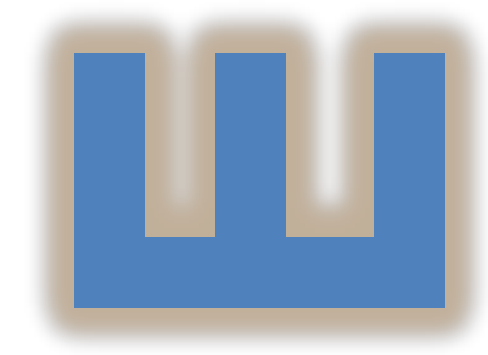

Figure 2-2 Schematic view from a cross section of the bulk density and the skeletal density of aerogel structure. The blue parts are the solid back bone (skeletal frame) and white parts between the skeletal frame are the pore spaces considered to be the pore volume.

The skeletal density of aerogel is the density for only the solid part of the aerogel structure, so the volume occupied by air in the bulk aerogel has to be excluded when it is measured Figure 2-2 shows the schematic definitions of the bulk density and the skeletal density. To pursue the measurement of the skeletal density, the total volume occupied by air in the aerogel structure 
needs to be accurately measured. This task can be accomplished by filling the pores with a measured amount of helium gas. There are two main reasons to use helium as a medium. First, helium does not dissolve into most solid materials, including solid silica. Second, the kinetic diameter of a helium molecule is the smallest among all inert gases as listed in Table 2-1 [67], so it minimizes the portion of the volume not measured due to any pores smaller in size than the medium gas. The kinetic diameter is the size of an atom or a molecule obtained by measuring the mean free path that the atom or molecule moves without colliding with other atoms or molecules [68]. It is different than atomic diameter defined by considering the size of the atom's electron shell $[69,70]$. The kinetic diameter reflects the actual space occupied by a single atom or a molecule. Table 2-1 lists kinetic diameters and atomic diameters of inert gases $[68,69,70]$.

Once the volume occupied by air is measured using this method, this volume is subtracted from the total measured sample volume and the mass of the sample is divided by this volume difference to obtain a skeletal density. This skeletal density can be measured by a helium pycnometer [71]. The helium pycnometer allows measuring the volume and true density of solid objects, without damaging samples. This is accomplished by employing Archimedes' principle of fluid displacement and Boyle's law to determine the volume. The displaced fluid is a gas (helium), which can penetrate the finest pores, thereby assuring maximum accuracy [72].

\subsection{Effective Medium Theory}

It has been reported that if the microstructure of a material is manipulated by introducing porosity, the Debye model may be inadequate to estimate the spectrum of continuum-like vibrational modes, since it is based on the average atomic density, and hence macroscopic sound velocity [73]. Therefore, the model of the minimum thermal conductivity [74] applied to fully 
dense solids, and the introduction of porosity thus require us to revisit this model. Instead, effective-medium theories for heterogeneous materials can be considered to gain insight into the thermal and mechanical properties [73]. The effective-medium theory is a method of treating a macroscopically inhomogeneous medium, i.e., a medium in which quantities such as the conductivity, dielectric function, or elastic modulus, vary in space [75]. The theory was first

Table 2-1. List of kinetic diameters and atomic diameters of inert gases $[68,69$, 70]. The atomic diameters are only available as calculated values except for the value for nitrogen. The atomic diameter of nitrogen listed in this table is a measured value [69].

\begin{tabular}{lcc}
\hline \hline & Kinetic diameter $(\mathrm{nm})$ & Atomic diameter $(\mathrm{nm})$ \\
$\mathrm{He}$ & 0.260 & 0.062 \\
$\mathrm{~N}_{2}$ & 0.364 & 0.155 \\
$\mathrm{Ne}$ & 0.275 & 0.076 \\
$\mathrm{Ar}$ & 0.340 & 0.142 \\
$\mathrm{Kr}$ & 0.360 & 0.176 \\
$\mathrm{Xe}$ & 0.396 & 0.216 \\
\hline
\end{tabular}

proposed by Eucken [76] and by Landauer [77] in a different context. Many materials fall into this broad category. One example is a metal-dielectric composite, consisting of a collection of metallic and dielectric grains arranged in some ordered or random fashion. Another example is 
a porous rock. If that rock is filled with salt water, then it is also a composite of an electrical insulator (the rock matrix) and an electrical conductor (the salt water) [78].

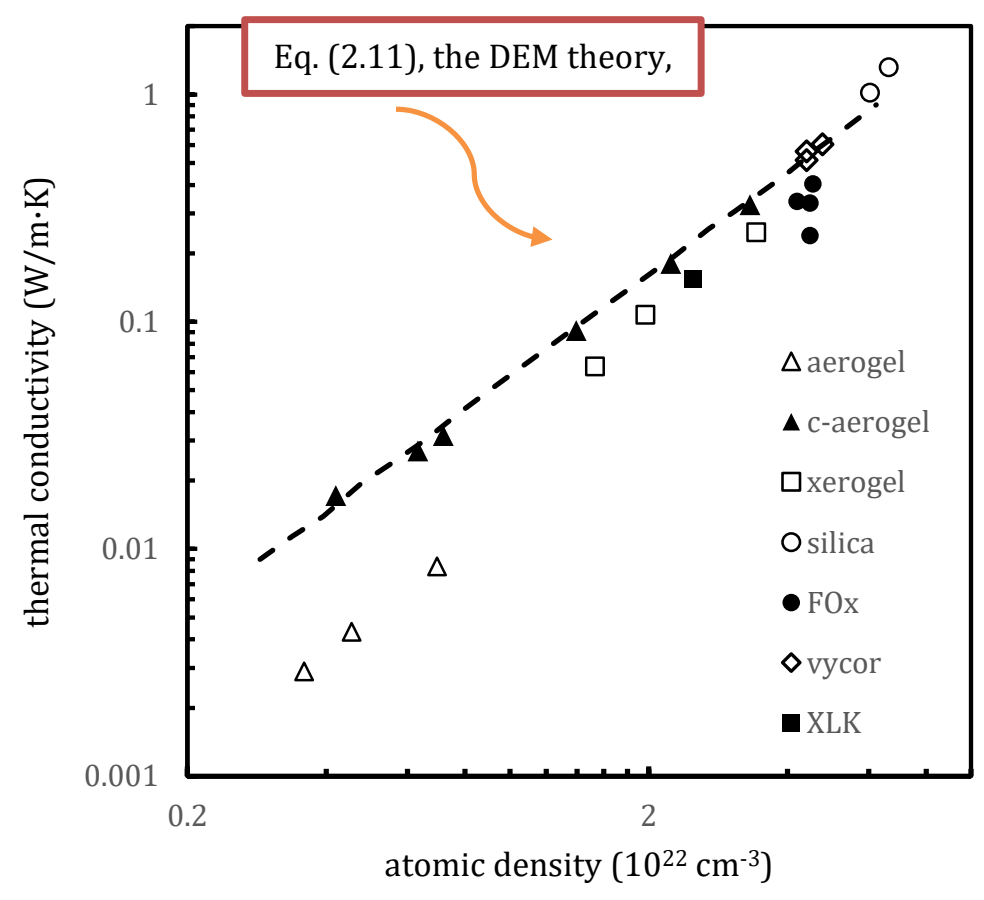

Figure 2-3. Sensitivity of thermal conductivity to the atomic density of $\mathrm{Si}$ and 0 for hydrogen-silsesquioxane films (solid circles labeled by FOx and XLK) and various forms of $\mathrm{SiO}_{2}$. This data was taken and replotted from reference [73]. For comparison, data for carbon aerogels (filled triangles), $\mathrm{SiO}_{2}$ (open circles), porous Vycor (diamonds), xerogel (open squares), and the lattice conductivity of silica aerogel (open triangles) are included. Data for $\mathrm{SiO}_{2}$ are from the literatures $[83,84,85,86,87]$. . The dashed line is the differential effective medium (DEM) theory [73].

When a composite material is constructed by making infinitesimal changes in an already exiting composite, the differential-effective-medium (DEM) theory has been reported as a more 
insightful method for describing the atomic density dependency of the thermal conductivity [73, $79,80,81,82]$. Considering an infinitesimal volume fraction of spherical voids in a composite, the thermal conductivity of the DEM theory, $k_{D E M}$, can be expressed as

$$
\frac{k_{D E M}}{k_{m}}=\left(\frac{n}{n_{m}}\right)^{1.5}
$$

where $k_{m}$ is the thermal conductivity of the composite matrix, $n_{m}$ is the atomic density of the composite matrix, and $n$ is the atomic density of the composite material with the voids. Costescu et al. reported that Eq. (2.11) from the DEM theory provides the best description of the dependence of the thermal conductivity data on atomic density among other effective-medium models as shown in Figure 2-3 [73, 83, 84, 85, 86, 87]. On the other hand, the downside of this theory is that knowledge of the bulk phase is required for the prediction. For example, in order to predict the thermal conductivity of aerogel films, prior knowledge of the thermal conductivity of bulk silica must be provided [73].

\subsection{Modified Model for Porous Materials with}

\section{Advantage}

Hopkins et al. later suggested modification of the minimum thermal conductivity for amorphous materials in order to describe that in a silica aerogel structure [88]. The original amorphous model was given by Cahill et al. as

$$
k_{\text {min }}=\frac{\pi}{3} \sum_{j} \int_{\omega_{j}} \hbar D_{j} v_{g, j}^{2} \frac{\partial f}{\partial T} d \omega
$$


where $\hbar$ is the reduced Planck's constant, $D$ is the density of states, $j$ is an index that refers to the polarization, $v_{g}$ is the group velocity, and $f$ is the Bose-Einstein distribution function. The density of states can be approximated as $D_{j}=\omega^{2} /\left(2 \pi^{2} v_{p, j}^{2} v_{g, j}\right)$, where $v_{p}$ is the phase velocity [89]. They modified the group velocity to scale with the number density in the overall volume of the sample as Eq. (2.12) has the assumption that the sound velocity can be described by the bulk velocities in $\mathrm{SiO}_{2}$ to describe thermal conductivity as a function of $\mathrm{SiO}_{2}$ atomic density [73]. This modification addresses the fact that sound velocity is reduced due to the porosity and resulting matrix of overlapping and multidirectional solid silica ligaments of the aerogel. The group velocities in silica aerogel scale by $\left(n / n_{b u l k}\right)^{1.4}$ according to Gross et al. [90]. By applying what has been considered, Eq. (2.12) is recast as a "porous minimum limit" given by Hopkins et al. [88]

$$
k_{\text {min }, \mathrm{p}}=\frac{1}{6 \pi} \sum_{j} \int_{\omega_{j}} \hbar \frac{\omega^{2}}{v_{\mathrm{p}, j}^{2}} v_{\mathrm{g}, j}\left(\frac{n}{n_{\mathrm{bulk}}}\right)^{1.4} \frac{\partial f}{\partial T} d \omega
$$

Compared to the DEM theory, the porous minimum limit model has remarkable advantage because it can predict the thermal conductivity of porous disordered materials without reference data for the corresponding bulk phase. Hopkins et al. demonstrated good agreement of the newly suggested model by plotting Eq. (2.13) along with the DEM theory prediction, and some examples of porous materials in Figure 2-4 $[88,91,92]$. Note that all of the data and predictions presented in Figure 2-4 taken from reference [88] represent the thermal conductivity through the solid silica network in the aerogel. Therefore, there is reasonable difference between what are displayed and the effective thermal conductivity of the silica aerogel [88].

While the overall degree of the agreement between the two models and the measured values is high in Figure 2-4, it is interesting to observe the differences in agreement between the aerogel samples. The predictions of the introduced theories show a better match at a range of higher 
atomic density. On the other hand, the thermal conductivity of the bulk silica aerogel with low atomic density remains quite different from that of both theories' predictions (approximately

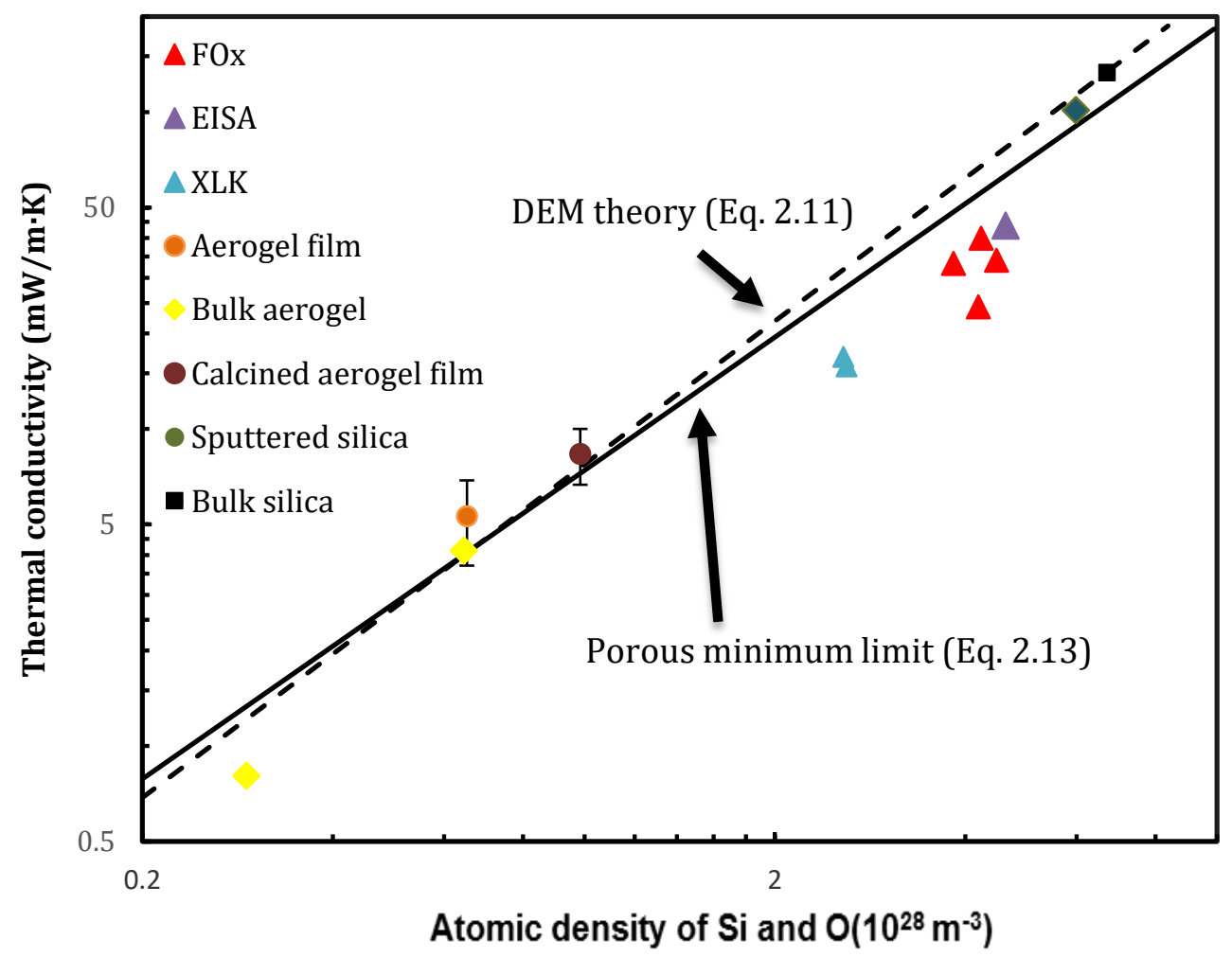

Figure 2-4. Effects of atomic density of $\mathrm{Si}$ and $\mathrm{O}$ on thermal conductivity for the aerogel and calcined-aerogel films along with bulk $\mathrm{SiO}_{2}$ [84], a sputtered $\mathrm{SiO}_{2}$ thin film [73], other porous silica materials (XLK and FOX) [73], and bulk SiO ${ }_{2}$ aerogels $[91,92]$. Predictions from the theoretical "porous minimum limit" to the thermal conductivity of $\mathrm{SiO}_{2}$ (solid line - Eq. 2.13) [88].

0.008 (at $10^{-4} \mathrm{mbar}$ ) vs $0.013 \mathrm{~W} / \mathrm{m} \cdot \mathrm{K}$ ). Rather, it shows better agreement with the thermal conductivity of the corresponding aerogel at near ambient pressure (approximately $0.014\left(\right.$ at $10^{2}$ mbar) vs $0.013 \mathrm{~W} / \mathrm{m} \cdot \mathrm{K}$ ). This relatively low agreement is caused by increasing degree of 
disorder of the silica network at a lower atomic density, which in general is related to the lower apparent density of the bulk aerogel. In Figure 2-4, the bulk aerogel with the higher atomic density has a density of $0.218 \pm 0.002 \mathrm{~g} / \mathrm{cm}^{3}$ and the one with the lower atomic density has a

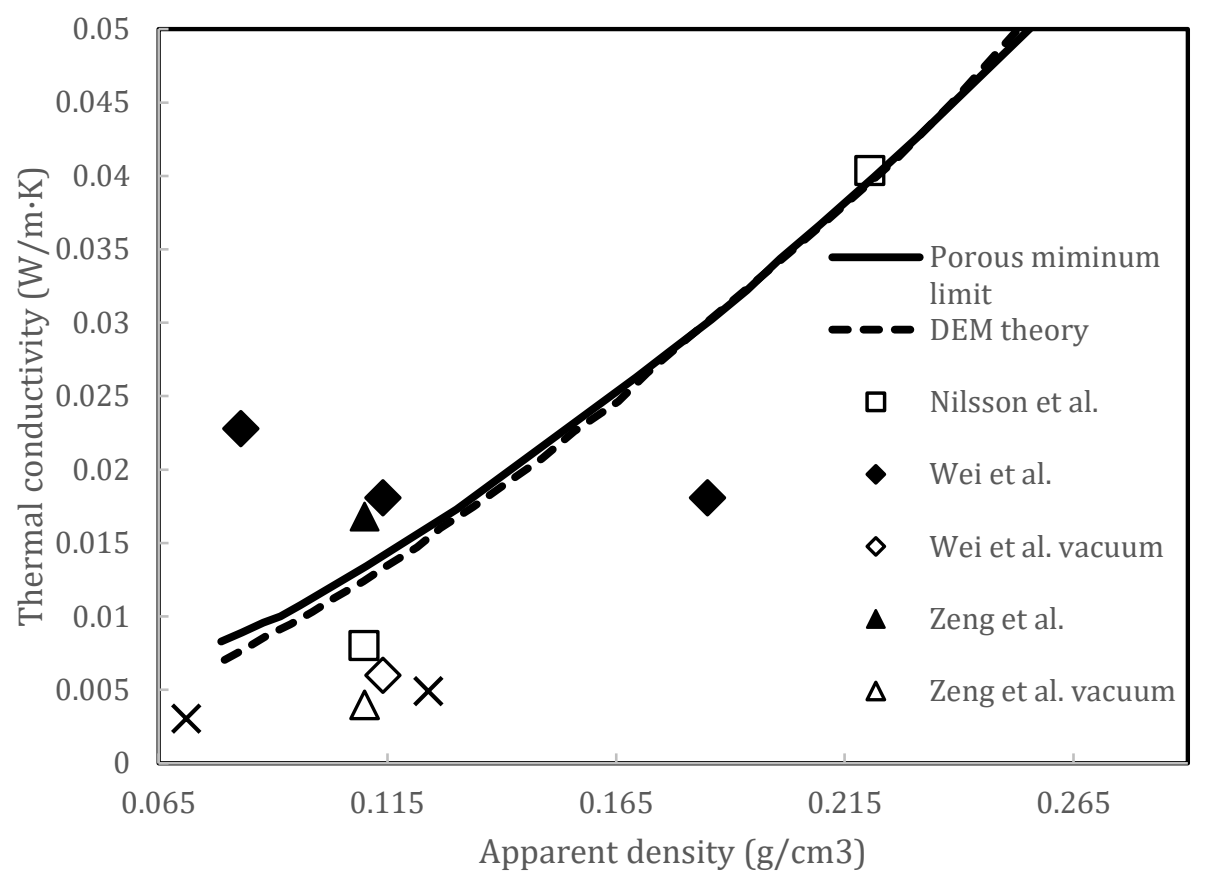

Figure 2-5. Thermal conductivities of silica aerogels reported with below 0.220 $\mathrm{g} / \mathrm{cm}^{3}$ of apparent densities $[87,93,94,95]$. The porous minimum limit (solid line) and the DEM theory (dashed line) were replotted from reference [88] by assuming the linear relationship between the atomic density of $S i$ and $O$ and the apparent density.

density of $0.110 \pm 0.004 \mathrm{~g} / \mathrm{cm}^{3}[91,92]$. Figure 2-5 shows additional thermal conductivities reported by different references that support what have been discussed [87, 93, 94, 95]. 


\subsection{Sensitivity of the Thermal Conductivity of}

\section{Aerogel to its Porosity}

This section introduces the available literature on how thermal conductivity is affected by pores in nanoporous materials and aerogels. The first sub-section (2.5.1) discusses results of the references for nanoporous silica. The next two sections (2.5.2 and 2.5.3) will present and discuss the data from the literature for carbon-opacified aerogels and organic-inorganic hybrid aerogels, respectively.

\subsubsection{Nanoporous silica}

Recall that the introduced methods that predict the thermal conductivity of silica aerogel focus on thermal conduction in the solid silica network. Sumirat et al. investigated the thermal conductivity of porous materials from a theoretical viewpoint with a focus on the influence of porosity [96]. Figure 2-6, taken from their study illustrates porosity dependence of thermal conductivity for porous materials. The vertical axis represents the thermal conductivity ratio, which is the ratio of the thermal conductivity at certain porosity to the value at zero-porosity. As plotted in Figure 2-6, the thermal conductivity of nanoporous materials depends on the ratio of the pore size $R_{p}$ to the phonon mean free path for zero-porosity, $L_{0}$. The thermal conductivity for $L_{0} / R_{p}>1$ decreases steeply with increasing porosity due to effective phonon scattering by pores. On the other hand, the thermal conductivity for $L_{0} / R_{p}<0.1$ decreases moderately with increasing porosity since phonon scattering by pores is no longer effective. Based on such predictions, they suggested the thermal conductivity of nanoporous materials depends on the 
pore size. If the average pore size is close to the phonon mean free path at zero-porosity, the phonon-pore scattering significantly reduces the thermal conductivity.

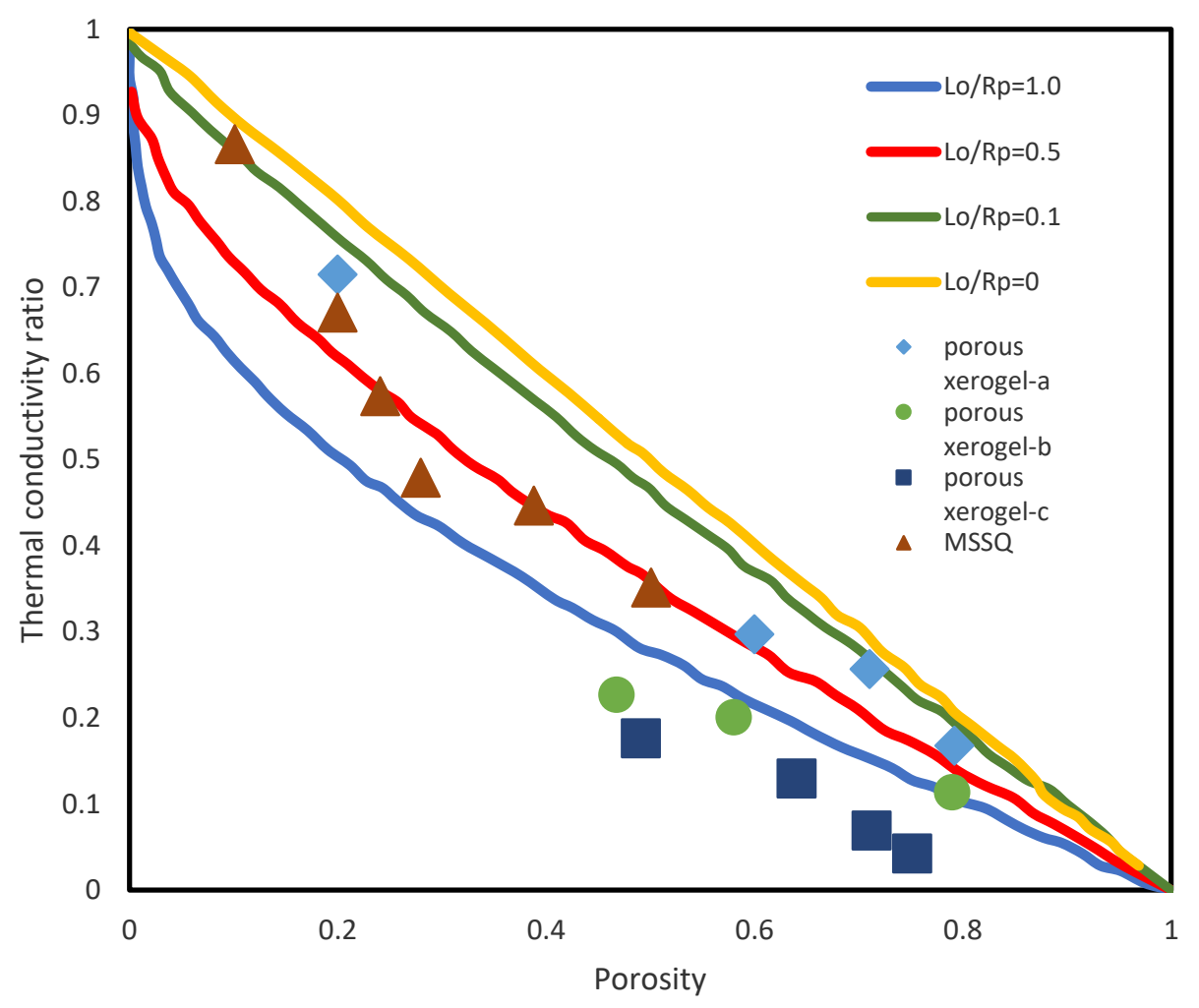

Figure 2-6. Summary of sensitivity of thermal conductivity to porosity for L0/Rp $=0.0,0.1,0.5$, and 1.0 in the present nanoporous model considered by Sumirat et al. and comparison with data experimentally reported. [96]. Porous xerogel$a$ is sintered silica xerogel film and porous xerogel-b is silica xerogel film made with a mixture of ethanol and ethylene glycol solvents [97]. Porous xerogel-c is a $\mathrm{SiO}_{2}$-capped xerogel film [86] and MSSQ is a porous organosilicate film formed by templated vitrification of copolymers consisting of polymeric matrix material of methylsilsesquioxane and porogens [98].

Sumirat et al. compared the theoretical description with the experimental data previously reported in Figure 2-6 [97, 86, 98]. Porous silica xerogel and MSSQ are $\mathrm{SiO}_{2}$-based nanoporous 
materials with pore sizes of $1-10 \mathrm{~nm}$. The phonon mean free path in single crystal $\mathrm{SiO}_{2}$ is about 3 to $5 \mathrm{~nm}$ at room temperature. Thus, the average pore size in the experimental samples is the same order of magnitude as the phonon mean free path at zero-porosity. This is the case for which phonon-pore scattering significantly effects the thermal conductivity. Experimental data in Figure 2-6 are in fair agreement with the curves for $l_{0} / R_{p}=0.5$ to 1.0 in the present model. Although this reported study was not for aerogel, it provides an initiative feel for the dependence of thermal conductivity on porosity in nanoporous materials, such as aerogels.

\subsubsection{Opacified silica aerogel}

Wei et al. synthesized opacified monolithic silica aerogels for use as thermal insulation in high temperature environments [92]. The aerogels were opacified with carbon nanofibers at different concentrations, as high as $20 \mathrm{wt} \%$, to enhance the dimensional stability of the silica aerogels and to lower the thermal radiation that increases thermal transport at high temperatures of $773 \mathrm{~K}\left(500{ }^{\circ} \mathrm{C}\right)$ and above. They obtained a thermal conductivity of $0.050 \mathrm{~W} / \mathrm{m} \cdot \mathrm{K}$ at $773 \mathrm{~K}$ $\left(500{ }^{\circ} \mathrm{C}\right)$ for these aerogels, which is lower than that observed in conventional thermal insulation materials $\left(0.3 \mathrm{~W} / \mathrm{m} \cdot \mathrm{K}\right.$ at $773 \mathrm{~K}\left(500{ }^{\circ} \mathrm{C}\right)$ for glass fibers, $0.1 \mathrm{~W} / \mathrm{m} \cdot \mathrm{K}$ at $800 \mathrm{~K}\left(527{ }^{\circ} \mathrm{C}\right)$ for alumina fused brick, and $1.7 \mathrm{~W} / \mathrm{m} \cdot \mathrm{K}$ at $800 \mathrm{~K}\left(527^{\circ} \mathrm{C}\right)$ for sillimantie). These results mean that they demonstrated successful incorporation of carbon nanofibers into the network of silica aerogels to enhance the dimensional stability of the silica aerogels, and to suppress the thermal radiations that become dominant at high temperatures [92]. In their experimental results, the thermal conductivities and porosities of the produced aerogels were tabulated from which the sensitivity of the thermal conductivity to the porosity can be estimated. 
Figure 2-7 shows the sensitivity of thermal conductivity of the sample aerogels to the apparent density (a) and the skeletal density (b) of the samples in their work, which were deduced based on the measured porosities and the apparent densities determined from,

$$
\text { skeletal density }=\frac{\text { apparent density }}{(1-\text { porosity })}
$$

Wei et al. report that the presence of the carbon nanofiber in the aerogel structure lowers the thermal conductivity as compared to pure silica aerogel. This improvement in thermal insulation is made possible thanks to the opacifier that retards the thermal transport in the aerogel structure by reducing the thermal radiation as expected $[99,100,101,102,103]$. Such results, however, are unexplained by simply considering the differences in the apparent density of the sample aerogels. For example, when the apparent densities of the produced samples are almost the same $\left(0.111 \pm 0.006 \mathrm{~g} / \mathrm{cm}^{3}\right)$, the thermal conductivities differ by $>10 \%$, ranging from $35-$ $38 \mathrm{~mW} / \mathrm{m} \cdot \mathrm{K}$, as shown in Figure 2-7 (a). These results show that the differing amount of opacifier in each aerogel $(13,15$, and $20 \mathrm{wt} \%)$ does not affect the apparent density as much as it does the thermal conduction. 


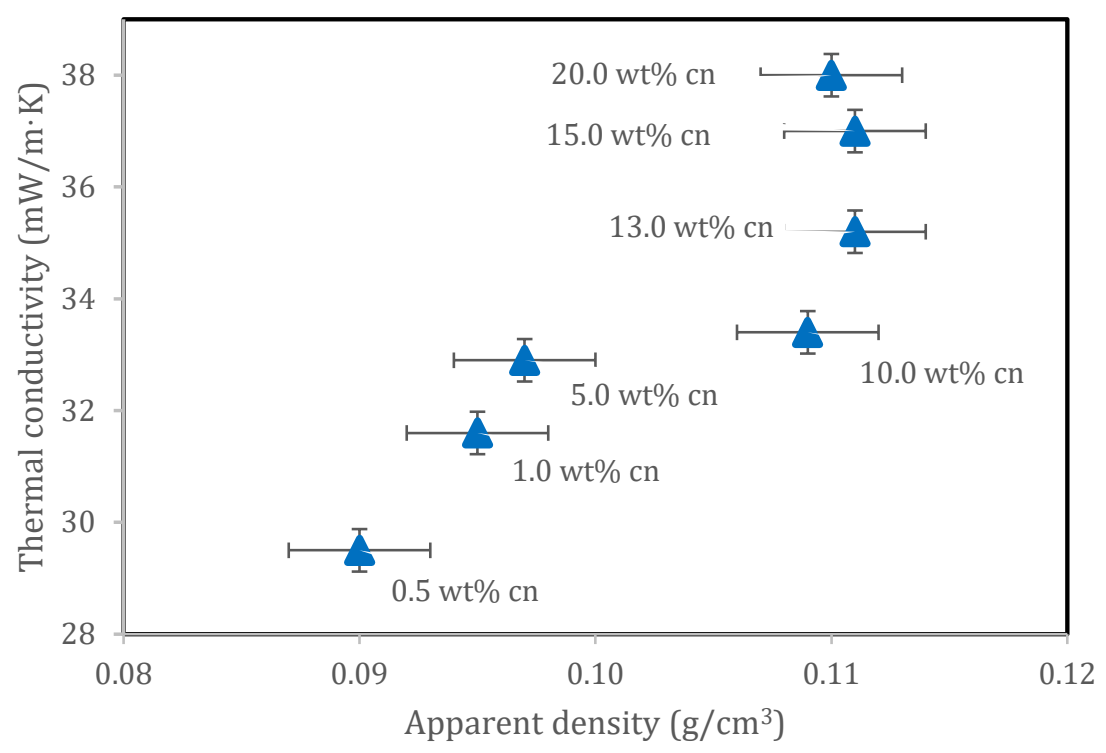

(a)

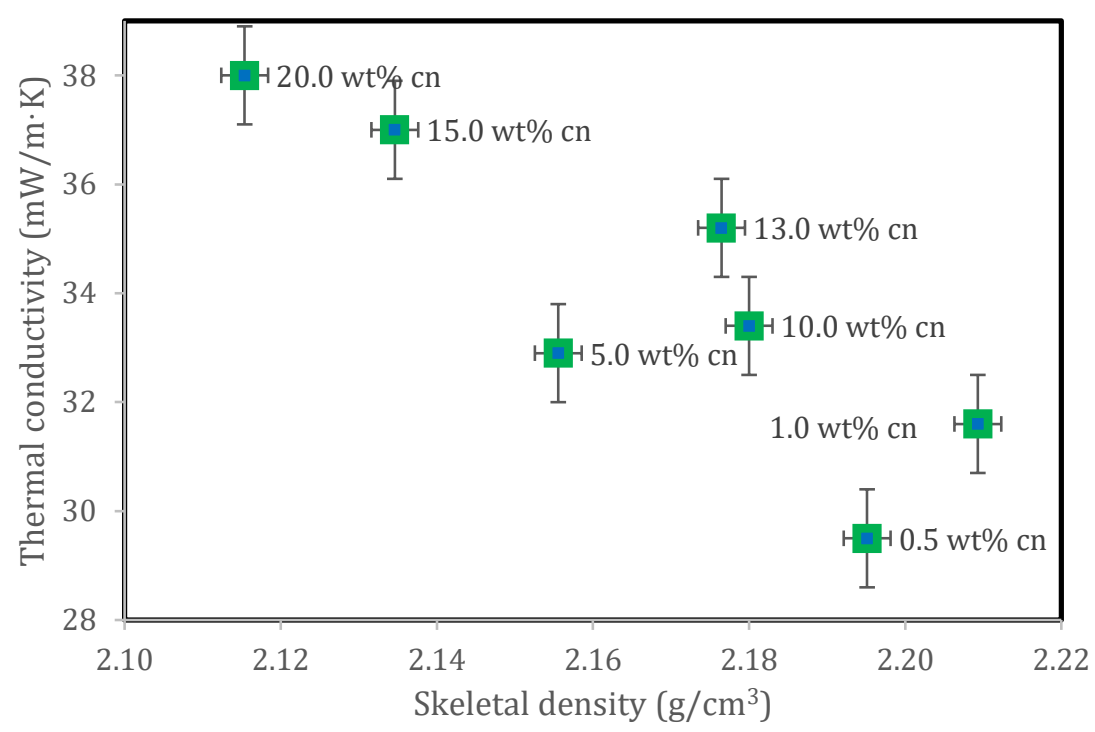

(b)

Figure 2-7. Illustration of sensitivity of thermal conductivity of opacified monolithic silica aerogel to apparent density (a) and skeletal density (b) at room temperature. It was arranged and plotted from the data originally presented in reference [92]. The data labels indicate weight pecentage of the opacifier that is carbon nanofiber (cn). 
On the other hand, one can recognize relatively clear differences in the skeletal densities between the aerogels discussed in Figure 2-7 (b). The skeletal density ranges from 2.135-2.209 $\mathrm{g} / \mathrm{cm}^{3}$ as the weight $\%$ of carbon nanotubes in the opacified aerogels ranges from $13-20 \mathrm{wt} \%$ in the same thermal conductivity ranges $(35-38 \mathrm{~mW} / \mathrm{m} \cdot \mathrm{K})$ observed previously. This sensitivity of thermal conductivity to the skeletal density is reflected and recognized when the

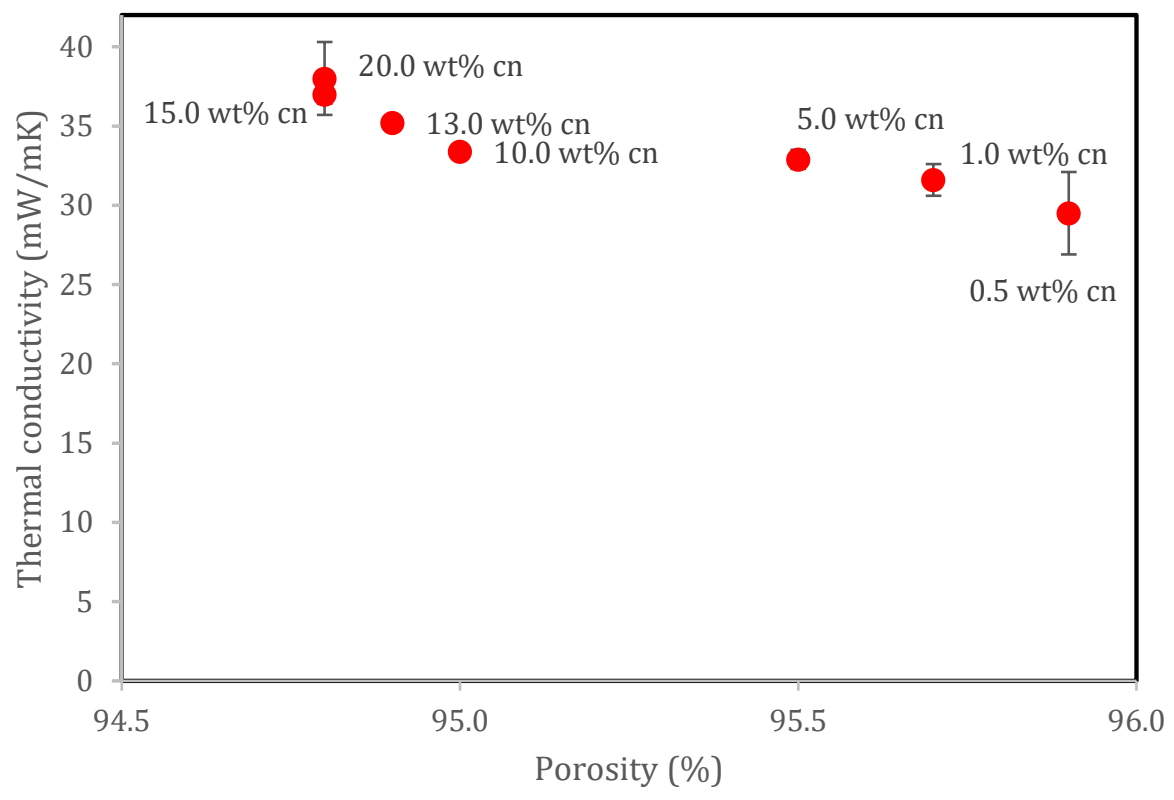

Figure 2-8. Sensitivity of thermal conductivity to porosity of opacified monolithic silica aerogel. It was arranged and plotted from the data originally presented in reference [92]. The data labels indicate weight pecentage of the opacifier that is carbon nanofiber (cn). The eror bars for 15,13 , and $10 \mathrm{wt} \% \mathrm{cn}$ are smaller than the data markers.

porosity is estimated, because the porosity is based on the relative ratio of the apparent density to the skeletal density of monolithic aerogel as introduced in Eq. (2.14). The thermal conductivity of the sample is inversely related to the porosity, as shown clearly in Figure 2-8. 
Therefore, it is possible that the change in the thermal conductivity can be predicted by only observing the porosity without considering the apparent density and the skeletal density separately.

\subsubsection{Hybrid organic-inorganic aerogel}

Nadargi et al. developed hybrid organic-inorganic silica aerogels by using methyltriethoxysilane (MTMS) as a precursor [66]. MTMS has one nonhydrolysable organic radical and the produced aerogel is an open cell polymer with an intricate internal structure and high porosity [104]. As the sample aerogels were prepared, they employed a typical two-step acid-base catalyzed sol-g el process. MTMS was diluted in methanol solvent with varying molar ratios of $\mathrm{MeOH} / \mathrm{MTMS}$ from 7 to 35 . The acidic water (oxalic acid, $0.001 \mathrm{M}$ ) was added to these solutions, drop by drop, while constantly stirring for $15 \mathrm{~min}$. In the second step, after 24 $\mathrm{h}$, basic water (ammonium hydroxide, $10 \mathrm{M}$ ) was added to the alcosol, drop by drop, again while stirring for the same time interval. The homogeneous alcosols thus obtained were transferred to airtight Borosil glass test tubes of $15 \mathrm{~mm}$ outer diameter and $150 \mathrm{~mm}$ height. Gelation took place under ambient conditions (temperature $\sim 27^{\circ} \mathrm{C}$ ). The prepared wet gels were placed in an autoclave for supercritical drying to transform them to aerogels. They used the HOT method by filling the autoclave with the mother solvent (methanol) and heating it to lower the capillary pressure $(P r)$ and hence the surface tension of the solvent to zero as per Laplace's equation [105],

$$
\operatorname{Pr}=-2 \gamma \cos \frac{\theta}{r}
$$


where $\gamma$ is the surface tension of the solvent, $\theta$ is the contact angle and $r$ is the pore radius. The temperature of the autoclave was raised to $265^{\circ} \mathrm{C}$ at a rate of $\sim 80^{\circ} \mathrm{C} / \mathrm{hr}$ and the corresponding pressure increased to $10 \mathrm{MPa}$ over $3 \mathrm{hr}$. Under these conditions, methanol was transformed into a supercritical fluid, which was evacuated from the autoclave, slowly, under isothermal conditions. The hydrolysis and condensation reactions are as per the chemical reactions in Figure 2-9 [106] and Figure 2-10 shows the resulting samples' densities and relation to thermal conductivities.

Hydrolysis:

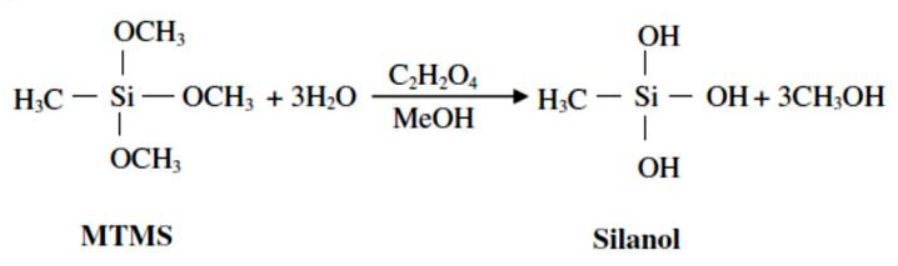

Condensation:

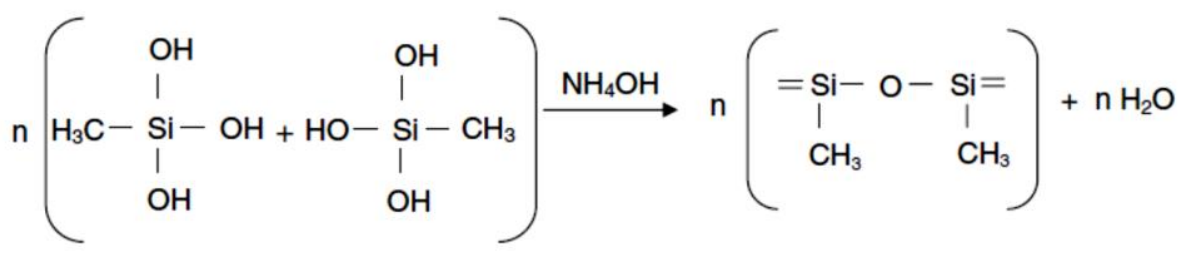

Figure 2-9. Hydrolysis and condensation route of the two-step sol-gel process for MTMS hybrid silica alcogel [106]. 


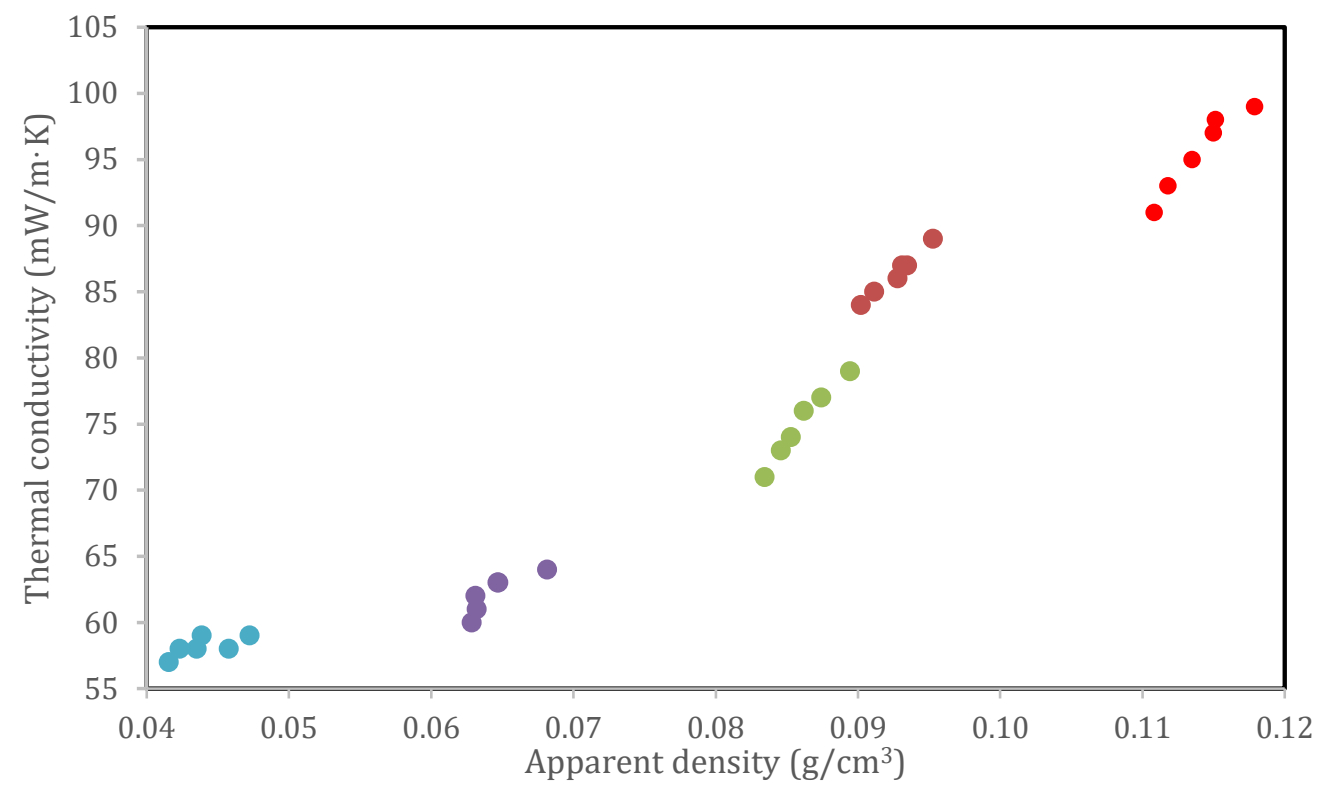

(a)

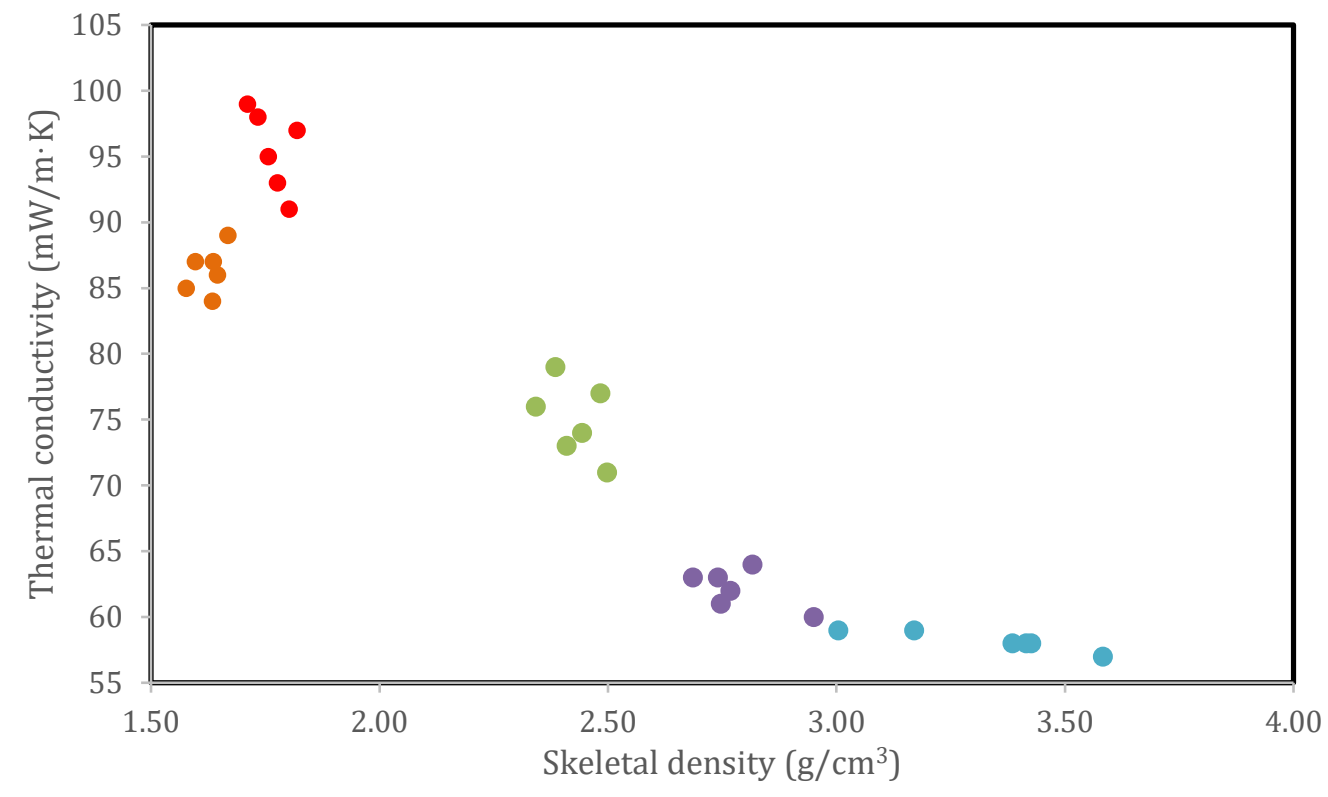

(b) 


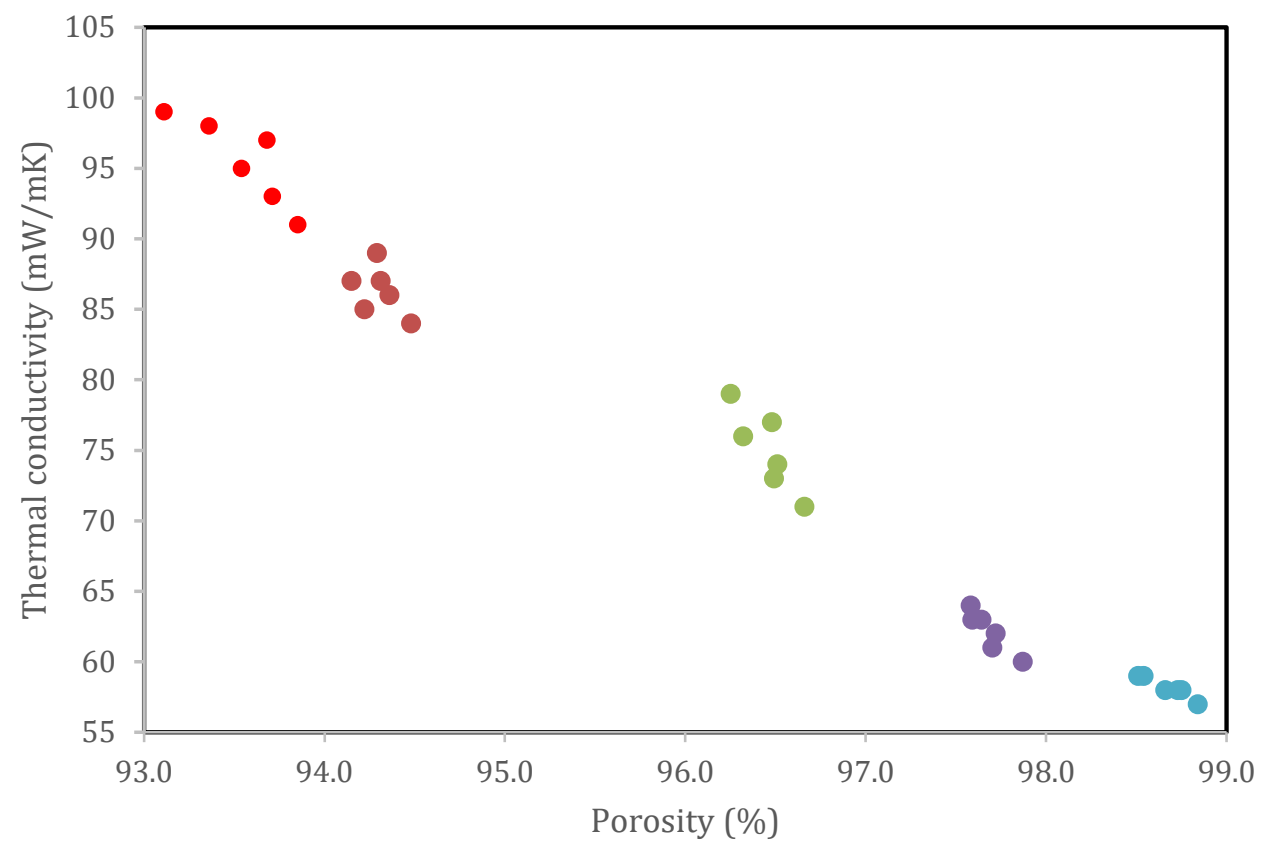

(c)

Figure 2-10. Illustration of sensitivity of thermal conductivity of MTMS hybrid silica aerogel to apparent density (a), skeletal density (b), and porosity (c) at room temperature. The sample aerogels prepared from the molar ratio of $\mathrm{MeOH} / \mathrm{MtMS}(\mathrm{M})$ $=7$ are marked as red dots, $M=14$ as brown dots, $M=21$ as light green dots, $M=28$ as violet dots, and $\mathrm{M}=35$ as light blue dots (arranged and plotted from Nadargi et al.'s work [66]).

As shown in Figure 2-10 (a) and (b), the thermal conductivity increases, nearly linearly, with apparent density, while decreasing, nearly linearly, with skeletal density, yet neighboring groups of different density are not always sufficiently distinguishable. For example, there is a clear overlap between the sample groups of $M=14$ and $M=21$ with the same apparent density (approximately $0.09 \mathrm{~g} / \mathrm{cm}^{3}$ ) but with thermal conductivity values from 80 to $84 \mathrm{~mW} / \mathrm{m} \cdot \mathrm{K}$ ) as shown in Figure 2-10 (a). We can also note from Figure 2-10 (b) that the thermal conductivity 
for the $M=14$ samples, which have a smaller skeletal density than the $M=7$ samples, is lower. This is in contrast to the other data for which the $\mathrm{M}$ values, and the skeletal density, are inversely proportion to the thermal conductivity (at $\mathrm{M}=21,28$, and 35).

All the above noted inconsistencies, however, are corrected and the data can be displayed in a much more organized way when the porosities of the sample aerogels are calculated and graphed with the measured thermal conductivity as shown in Figure 2-10 (c). The thermal conductivity decreases when the porosity increases over the range studied similar to that which Nadargi et al. reported [66]. In addition, the inconsistency of the density differences along with the thermal conductivity changes are well explained when the porosity and the thermal conductivity are paired, which means the formation of the silica backbone and the pores during the sol-gel step are not only affected by the target density but by multiple parameters.

As discussed, the skeletal density and the apparent density of monolithic aerogel are intrinsically related and this relationship can be understood in a more straightforward way when porosity is included in the considerations, as the porosity is, in a way, a combination of the two properties. The thermal conductivity of the aerogel is furthermore quite sensitive to the porosity, which can be used as a key parameter to estimate the resulting thermal conductivity of produced aerogel once the designated sol-gel process is employed. 


\subsection{Research Objectives}

The global aerogel market size was estimated at USD 352.6 million in 2014. At that time, it was stated that the market was likely to witness significant growth over the next seven years owing to its rising demand from various end-use industries as shown in Figure 2-11 [107]. The growth of these end-use industries was expected to further fuel growth over the forecast period [107].

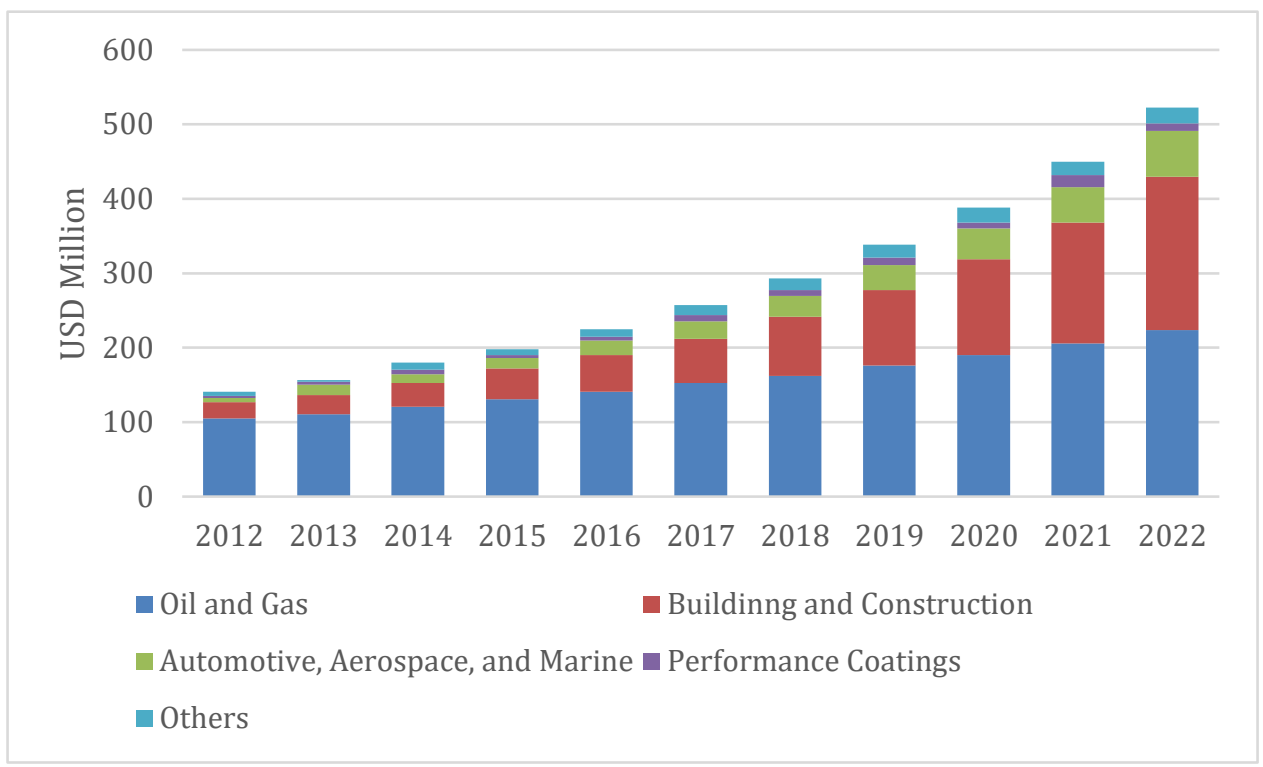

Figure 2-11. U.S. aerogel market revenue by end-use, 2012-2022, The data were taken from Aerogel market by raw material (Clark, Allied Market Research, 2016 [107]).

Such explosive growth requires a large production system of aerogel via an appropriate pilot plant test as a typical scale up procedure. In addition, continuous efforts to reduce the production costs by optimizing and updating the existing processes are challenging key elements to stay successful in the competitive industry. Due to the nature of the aerogel production process, the initial development of the chemical production process, which is sol-gel synthesis 
in many cases, is initially pursued at a lab scale of $\mathrm{ml}$ and then scaled up to a plant scale of several hundred gallons. Therefore, extremely fine adjustments are necessary at the lab scale step with a focus on the target property of the final aerogel product.

Table 2-2. Example of developing aerogel process by screening sol-gel parameters with regards to best thermal insulation performance for scale up to a pilot plant (Aspen Aerogels, Inc.). Note that the process (yellow-highlighted) that produced TEHY-1 was selected as the optimized production process because its thermal conductivity is the lowest one among other candidates. The next lowest one, TEHY-2, has higher thermal conductivity only by $0.7 \mathrm{~mW} / \mathrm{m} \cdot \mathrm{K}$. but not selected. (a : hydrolysis time to prepare a sol, $b$ : apparent density, $c$ : thermal conductivity)

\begin{tabular}{|l|l|c|c|c|c|c|}
\hline \multirow{2}{*}{$\begin{array}{l}\text { Formulation1 } \\
\text { Sol A }\end{array}$} & sample ID & TEHY-1 & TEHY-2 & TEHY-3 & TEHY-4 & TEHY-5 \\
\cline { 2 - 7 } & $\begin{array}{l}\text { Unopacified } \\
\text { (hour) }\end{array}$ & 3.88 & 4.25 & 6.05 & 22.5 & 29.5 \\
\cline { 2 - 7 } & $\begin{array}{l}\text { gel time } \\
\text { (minute) }\end{array}$ & 2.16 & 2.083 & 2.16 & 1.83 & 1.97 \\
\cline { 2 - 7 } & $\begin{array}{l}\text { Density } \\
\text { (g/cc) }\end{array}$ & $\mathbf{0 . 1 0 6}$ & $\mathbf{0 . 1 0 0}$ & $\mathbf{0 . 0 9 6}$ & $\mathbf{0 . 0 9 9}$ & $\mathbf{0 . 1 0 1}$ \\
\cline { 2 - 7 } & $\begin{array}{l}\mathbf{k}^{\mathrm{c}} \\
\text { (mW/mK) }\end{array}$ & $\mathbf{1 2 . 3}$ & $\mathbf{1 3 . 0}$ & $\mathbf{1 4 . 0}$ & $\mathbf{1 3 . 6}$ & $\mathbf{1 4 . 1}$ \\
\hline & hydrophobicity & yes & yes & yes & yes & Yes \\
\hline
\end{tabular}

Table 2-2 shows an example of the aerogel process development utilizing a screening of sol-gel parameters to optimize performance for thermal insulation for scale up to a pilot plant at an actual company that has been in the aerogel market for over a decade. Aspen Aerogel, Inc. produces aerogel composites for thermal insulation in various applications so the thermal conductivity of their product is a key property. The "best" process is selected by comparing the thermal conductivities of the samples prepared by sol-gel processes nominated from a 
preliminary screening. Even after the preliminary screening, however, they had to try a certain range of sol-gel conditions one by one. They only knew the apparent density from each process and attempted to use this information to find the lowest thermal conductivity, but there is no consistent trend between the apparent density and the thermal conductivity as seen in Table 2-2. The only way to ensure the best process is identified is for all nominated processes to be experimentally produced, yielding aerogel samples that satisfy the requirements in size and shape, and then the resulting thermal conductivities must be measured and compared

For the example given, the best process produced a sample named TEHY-1 and its thermal conductivity is the lowest among other produced samples $(12.3 \mathrm{~mW} / \mathrm{m} \cdot \mathrm{K})$. The next lowest thermal conductivity was obtained from TEHY-2, but this sample was not selection for production due to the only $0.7 \mathrm{~mW} / \mathrm{m} \cdot \mathrm{K}$ higher thermal conductivity $(13.0 \mathrm{~mW} / \mathrm{m} \cdot \mathrm{K})$. This example demonstrates the accuracy they had when comparing the outcomes from each process to deliver the most efficient process to the scale up stage.

On the other hand, such a process investigation could have been pursued more efficiently if the relationship between the porosity and the thermal conductivity of aerogel had been recognized. They could identify the target density that maximizes the porosity. This could be simply done by producing small monolithic aerogels from the nominated processes and measuring apparent densities and skeletal densities to obtain porosities. The best process could have then been defined as the one that produces the sample with the maximum porosity, as demonstrated in this chapter. Also, this optimization of the aerogel production process can be more simplified if the employed sol-gel process has high feasibility in implementation for large production system in terms of high controllability of the process parameters and low production cost. 
With such an understanding of current circumstances in the aerogel industry, the objectives of this dissertation have been defined as follows:

\author{
1. Development of a sol-gel process that is highly feasible to scale up for \\ industrial implementation of large production and for controlling the key \\ properties controlling thermal conductivity of produced monolithic silica \\ aerogel.
}

2. Study the effects of the developed sol-gel parameters and resulting properties on the porosity of the produced monolithic silica aerogels.

3. Study the effects of textural properties on the porosity of produced monolithic yttria aerogels.

4. Study the effects of textural properties on the porosity of produced monolithic organic aerogels.

5. Demonstration of the 3-omega method by the planted wire set into produced monolithic aerogels to measure thermal conductivity.

\title{
2.7 Brief Outline of Following Chapters
}

Chapter 3 will describe the new production route developed in this research, the directcontact method, DCM. This development was important because it enables production of silica aerogels of various properties from a single preparation of the intermediate product (prehydrolyzed sol). It also increases the feasibility of large production of silica aerogels at lower cost than the conventional process for industrial applications. Then in Chapter 4 , the results from 
a study of effects of process parameters on structural properties of silica aerogel, including the porosity, will be provided and important conclusions will be drawn. It will be demonstrated that the chemical parameters in the aerogel production process affect the porosity even when the physical parameters are set to be identical. The results from the basic study of porosity of yttria and organic aerogels will be reported in Chapter 5. Effects of target density on porosity and structural properties of yttria and organic aerogels will be introduced for the first time. Then in Chapter 6 results of the thermal conductivity measurement of the produced aerogels are presented by the first set of attempts of the 3-omega method with an implanted wire-set into the monolithic samples. Finally, Chapter 7 will present conclusions focused on the above objectives and close with recommendations for further work regarding the thermal conductivity measurement by the 3 -omega method to find the actual thermal conductivity change with that of the porosity of the produced aerogels. 


\section{Chapter 3}

\section{New Sol-Gel Route for Silica Aerogel Synthesis: Direct-Contact Method (DCM)}

This chapter introduces a new sol-gel process, the direct-contact method (DCM) (U.S. Provisional Patent Application Serial No. 62/486,057 filed April 17,2017). This production route has been developed based on the two step sol-gel process to synthesize a silica gel. In the DCM, the pre-hydrolysis step is modified to increase controllability of the processing conditions to tailor the final product as various types of silica aerogel. Details and discussion of this new sol-gel method are provided after motivation for the development is first given. Finally, the differences between this new process and the conventional process will be summarized, and the benefits of using it will be presented.

\subsection{Motivation for Process Development}

Over the last two decades, the two step sol-gel process has been broadly used to produce silica aerogel because the hydrolysis and the poly condensation of the sol-gel reactions can be separately controlled by using an acid catalyst and a base catalyst respectively. It is important to control the two reaction rates relatively to synthesize a polymeric wet gel, as a gel is formed only when the relative reaction rate of the condensation is considerably higher than that of the hydrolysis (Figure 3-1 [108]). 

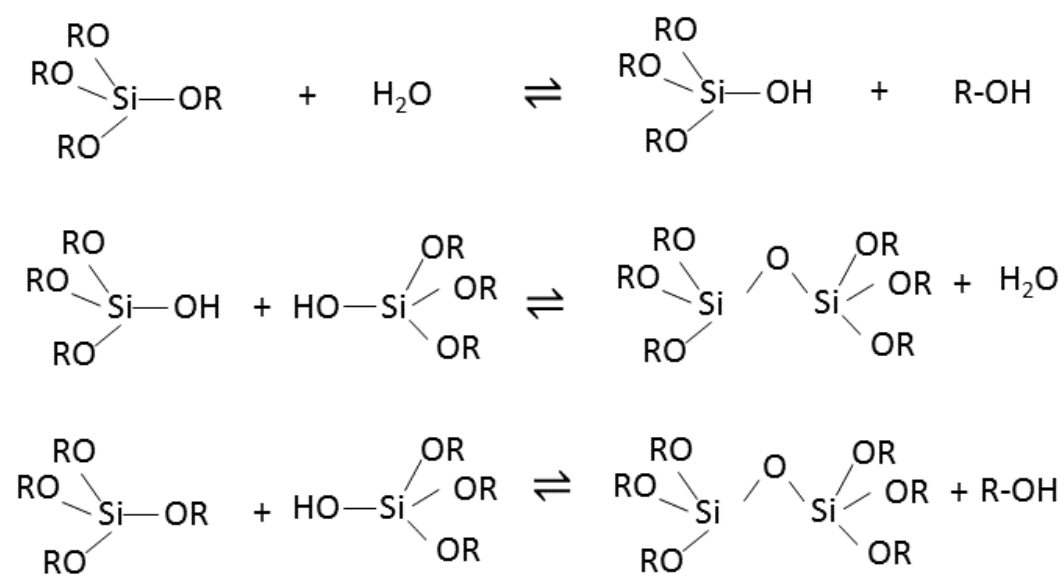

Figure 3-1. The three main reaction steps occurring in the two step sol-gel process of alkoxysilanes: (1) hydrolysis of an alkoxy group produces silanol groups and the respective alcohol; (2a) condensation of two silanol bearing species; and (2b) mixed condensation of a silanol group and an alkoxy group bearing species [108].

The conventional method for hydrolysis of silica precursor in the two step acid-base catalysis system includes diluting the precursor in its mother liquor prior to adding understoichiometric amounts of water and an acid catalyst to form pre-hydrolyzed silanol groups $\left[\mathrm{Si}(\mathrm{OR})_{3}(\mathrm{OH})\right]$ and silica sol $\left[\mathrm{Si}(\mathrm{OH})_{4}\right][109]$. However, this method, in many cases, is time extensive and an energy-consuming process. The solution needs to be heated up and cooled down to enhance the hydrolysis rate and to stabilize the state of the sol, respectively. This approach also yields relatively slow gelation due to the low concentration of the active silanol groups by only partial hydrolysis of the precursor. While it is common for most metal alkoxides to have high reaction rates for hydrolysis, the uncommonly low hydrolysis reaction rate of silicon alkoxide is a disadvantage when a silica sol-gel reaction is considered as the production process for any silica products. The extra amount of water and solvent lower the silica content in the produced sol batch and thus also increase the production costs, as this limits the concentration of the final product. Also, the adaptability of the conventional sol-gel process to other processes which might be used to produce hybrid materials or to co-gel with other species 
is low, so this must be considered as the diversity of the types of sol-to-gel products desired for various applications increases rapidly.

\subsection{Direct-Contact Method (DCM)}

Presented here is a new process for producing aerogels, both silica and hybrids. In this new process, TEOS or TMOS is pre-hydrolyzed by the precursor-water direct-contact method (DCM) in order to control the gelation time across a broad range from a few minutes to hours. The idea of the DCM is to let the precursor and water mix without an additional diluent so the contact rate of water and silane groups increases. However, this was previously known to be only theoretically possible due to the poor miscibility of the silicon alkoxide with water, which also results in very low reactivity towards hydrolysis reactions even in the presence of excessive stoichiometric amounts of water. Figure 3-2 (a) briefly shows the differences between the main concepts in the conventional two step sol-gel process and the DCM.

\subsubsection{Description of the developed process}

In order to overcome the difficulty resulting from the poor miscibility of the silicon alkoxide with water in silica sol-gel chemistry and in order to produce silanol groups [silica sol, $\left.\mathrm{Si}(\mathrm{OR})_{3}(\mathrm{OH})\right]$ which are polymeric and homogeneously formed, the $\mathrm{pH}$ of the reaction system needs to be adjustable as the hydrolysis reaction proceeds. The silicon alkoxide (TEOS in this case) shows relatively high reaction rates of hydrolysis at strong acidic conditions (at or below 

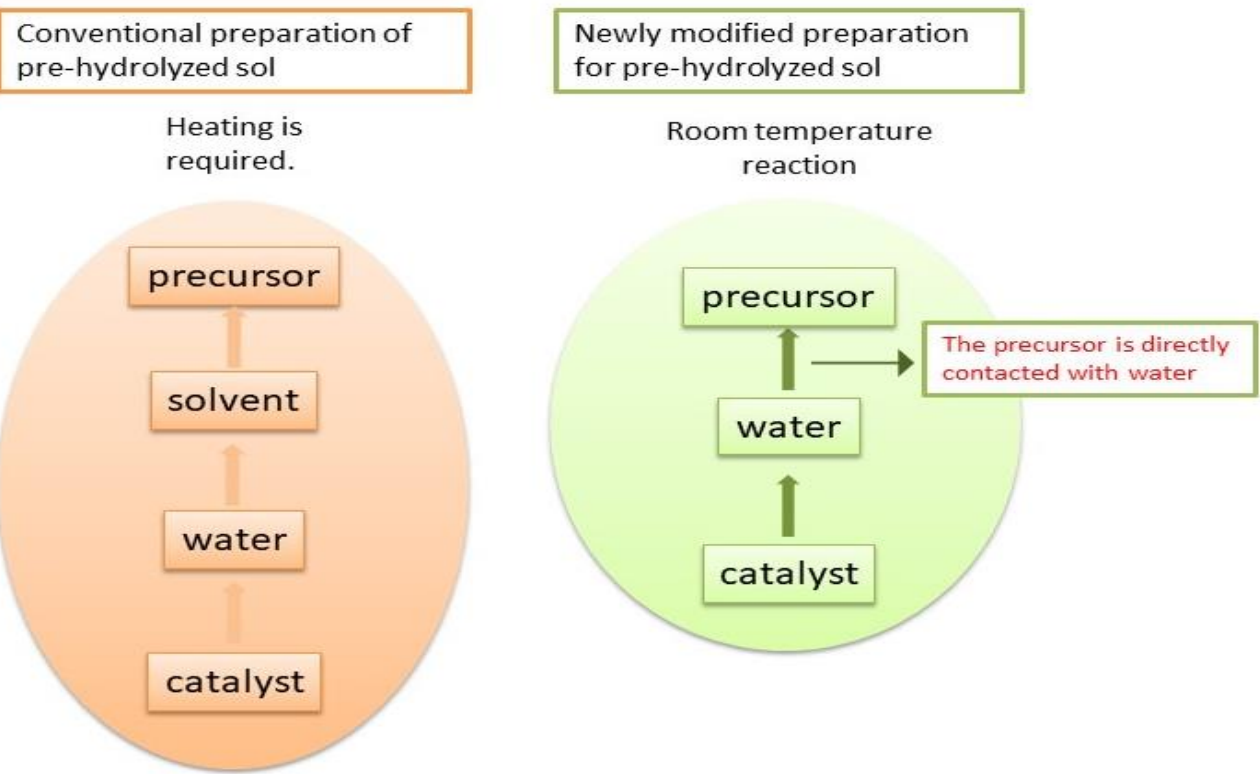

(a)
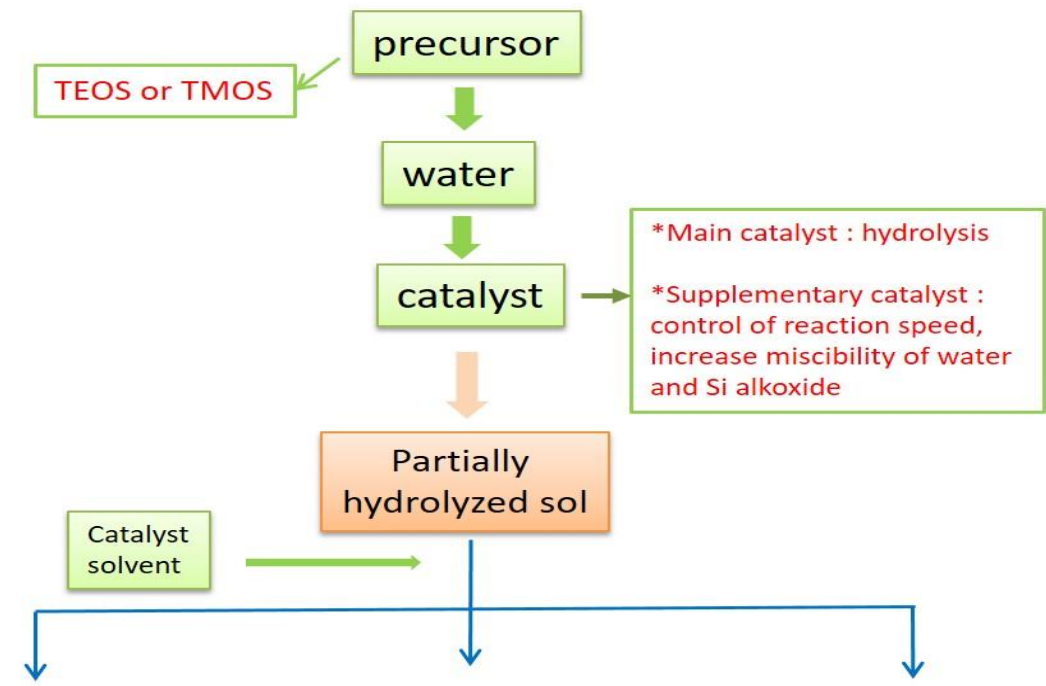

Film Polymeric gel

Colloidal gel

(precipitate)

(b)

Figure 3-2. (a) Schematic comparison of conventional pre-hydrolysis and the direct-contact method (DCM) hydrolysis. (b) Example of the two step sol-gel process by employing the DCM and resultant products. 
$\mathrm{pH}$ 2.0) and strong basic conditions (at or near $\mathrm{pH}$ 14.0). Based on this property, the DCM was initiated by sequentially applying a dual catalyst system of strong acid as the main catalyst and weak acid as a supplementary one. The strong acid catalyzes the hydrolysis reaction while the weak acid drives a gradual change of the $\mathrm{pH}$ so the hydrolysis occurs homogeneously and simultaneously in the reaction system. The control of the $\mathrm{pH}$ change also increases the miscibility of the TEOS and water by releasing negative ions that promote the deprotonation of the water. This results in hydrolysis within a few hours when the catalysts are added. In addition, the higher concentration of Si in the pre-hydrolyzed sol yields a wider range over which we have the ability to control the target density of the synthesized gel.

Figure 3-2 (b) shows the whole sol-gel pathway when the conventional two-step process is replaced with the DCM and the possible types of final products resulting when the gelation proceeds under appropriate conditions. When the final product is a film or a colloidal gel, the water amount is adjusted at under and over-stoichiometric ratios to the precursor, respectively. This adjustment can be conveniently made in the pre-hydrolysis step or the gelation step in the DCM. Gel films, in many cases, are produced on a substrate and this requires a certain portion of open chain hydroxyl (-OH) groups where durable bonding is made between the substrate (often glass or boron silicate) and the film layer. Such hydroxyl groups are obtained by reacting the silica precursor with under-stoichiometric amounts of water in the hydrolysis process so that the condensation is incomplete with some quantity of silanol remaining unreacted. The colloidal gel is produced when the hydrolysis rate is overwhelmingly increasing relative to the condensation rate during the sol-to-gel step. This is mainly caused by providing overstoichiometric amounts of water and the final product is the result of precipitation, due to the early termination of gelation before the silica network is maximized to the entire volume of the reaction system (solvent). This also causes irregular formation of the silica network in size and shape. The polymeric gel is made when the hydrolysis is driven by stoichiometric amount of 
water that is accurately adjusted. This hydrolysis produces a clear silica sol, which means the sizes of the hydrolyzed sols are smaller than what is influenced by van der Walls forces. This small size enables homogeneous distribution of the silica sols throughout the reaction system (the liquid batch where the hydrolysis occurs). When the hydrolysis is nearly completed, the condensation reaction is driven to connect the silica sols to form the silica networks. These silica networks are crosslinked three dimensionally, and result in highly homogeneous and open pores between them.

\subsubsection{Selection of catalysts}

The most important step in development of the DCM is patient investigation of the potential combinations of acid catalysts. The ideal combination preferably meets the following criteria:

1. Sufficient increase in the miscibility of the silica alkoxide and water without a solvent.

2. Drives homogeneous hydrolysis at room temperature.

3. The produced sol is ready for the next step (gelation) within the time that is appropriate for process implementation regardless of its scale.

4. The produced sol has reasonable pot life time so that it can be stable until the entire solgel process is completed.

5. The selected catalysts are inexpensive and broadly used so there are no safety issues that arise. 
The combination of the catalysts can be made as a pair of strong acid-weak acid as discussed earlier. Having the above conditions considered, a series of preliminary investigations were pursued for this work. Hydrochloric acid, nitic acid, and sulfuric acid were down selected as potential strong acids, and acetic acid was exclusively selected as the weak one to efficiently investigate the formulation trial. Pairs of nitric acid-acetic acid and sulfuric acid-acetic acid were further selected in this dissertation research for the next round of investigation, where the relative molar ratios of the paired catalysts, silica precursor (TEOS), and water were adjusted. Hydrochloric acid was excluded in this primary research due to an expected concern with its fumes and its high corrosiveness when the scale-up attempt is made. An additional investigation on the use of hydrochloric acid will be proposed as one of the future works introduced in the final chapter.

\subsubsection{Quantitative definition of each composition}

Error! Reference source not found. shows the resulting ranges of each composition of the DCM where the homogeneous sol was synthesized. While these ranges are defined to be employed to produce monolithic silica aerogel, it is confidently believed that a much broader

range in molar ratio can be defined for the same compositions when other types of gels need to be produced.

The adjusted molar ratios dynamically affect the reaction time, the pot life time, the morphology of the gel via gelation, and most importantly the porosity of the produced aerogel. When the molar ratio of water is used at a value close to the lower limit (2.00), homogeneous hydrolysis occurs earlier but the gelation time increases under the same condition because fewer silanol groups are involved in the poly condensation (gelation). Use of lower concentrations of catalysts (lower limit in Error! Reference source not found.) requires longer reaction times to 
reach the homogeneous hydrolysis but results in longer pot life time so the produced sol can be stored and available to produce gel products for longer periods of time.

Table 3-1. Investigation results on optimized relative molar ratio of involved compositions in the DCM.

\begin{tabular}{lcccc}
\hline $\begin{array}{l}\text { Involved } \\
\text { composition }\end{array}$ & TEOS & water & $\begin{array}{c}\text { nitric acid } \\
\text { (sulfuric acid) }\end{array}$ & acetic acid \\
\hline Relative & 1 & $2.00 \sim 4.83$ & $5.0 \times 10^{-4} \sim 1.0 \times 10^{-3}$ & \\
molar ratio & & & $\left(6.0 \times 10^{-4} \sim 1.2 \times 10^{-3}\right)$ & $2.0 \sim 3.5 \times 10^{-2}$ \\
& & & & \\
\hline
\end{tabular}

Throughout the investigation process, it was noted that the relative molar ratio of the combination of catalysts was crucially important as they react with each other, as well as the remainder of the reactants. Only certain ranges of the molar ratio used in this research enabled the miscibility of water with the silica alkoxide (TEOS) to be increased and reach the homogeneous hydrolysis where the milky mixture becomes clear. When the molar ratio is outside the optimum range, the hydrolysis occurs inhomogeneously so the solution either remains cloudy or it is once clarified but returns to a cloudy state due to failure of $\mathrm{pH}$ control by the catalysts. In this research, the molar ratio of nitric acid - acetic acid combination and sulfuric acid - acetic acid combination were adjusted to 0.029 and 0.035 , respectively.

\subsubsection{Observation of the DCM process}

There is a dynamic change in temperature and $\mathrm{pH}$ of the solution when the silica alkoxidewater solution is catalyzed for homogenous hydrolysis by the DCM as discussed previously. 
Figure 3-3 and Figure 3-4 illustrates how the hydrolysis proceeds in the DCM by profiling $\mathrm{pH}$ and temperature for the first three hours for the nitric acid-acetic acid combination and the sulfuric acid-acetic acid combination, respectively.

In the case of the combination nitric acid-acetic acid, Figure 3-3, the initial $\mathrm{pH}$ (solid triangle on red line) is about 2.2 and it decreases to a little below 2.1, but then returns back to 2.15 with a gradual increase in temperature (yellow $\mathrm{X}$ on blue line) of approximately $5^{\circ} \mathrm{C}$ from

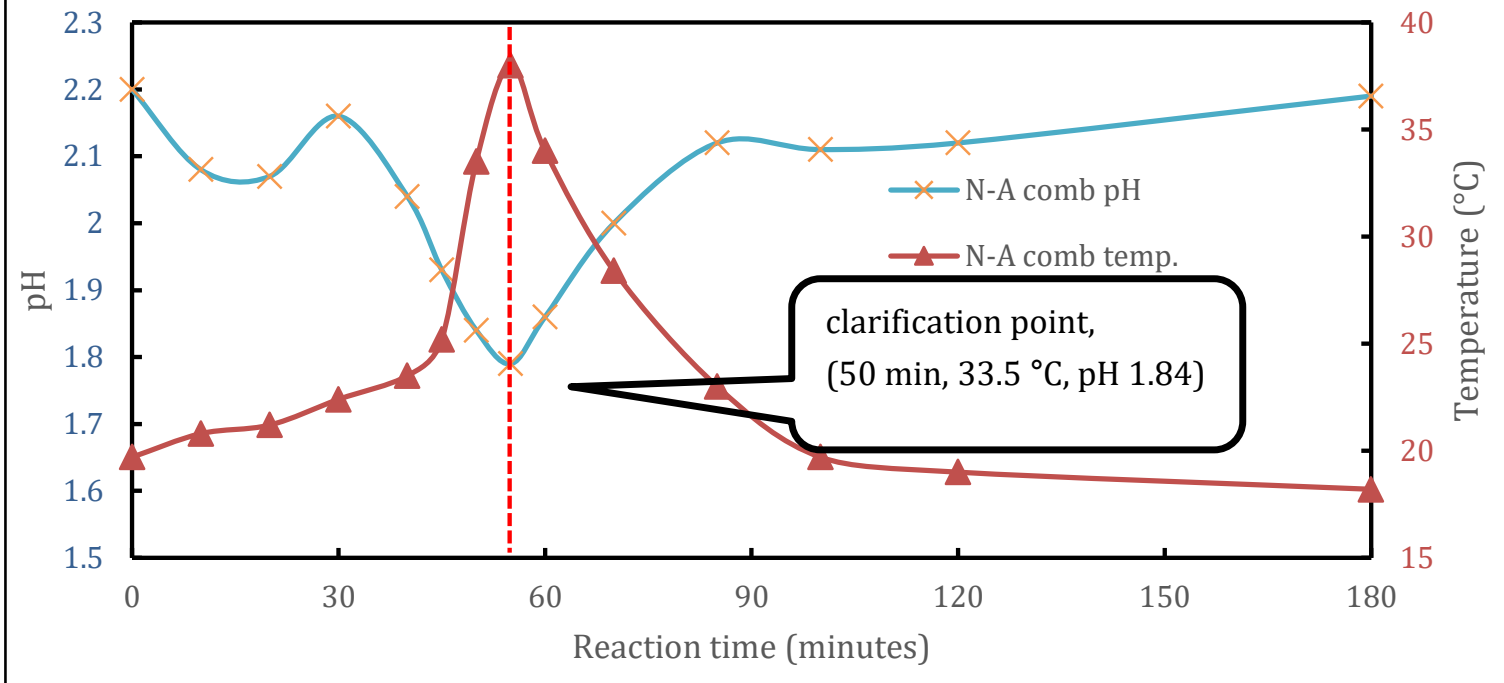

Figure 3-3. pH and temperature profile of the hydrolysis reaction of TEOS by the directcontact method (DCM) when nitric acid and acetic acid catalyzed the hydrolysis. The molar ratio of TEOS : water : nitric acid : acetic acid is $1: 4.83: 1.0 \times 10^{-3}: 3.5 \times 10^{-2}$, respectively.

room temperature over the first 30 minutes. After this period, the $\mathrm{pH}$ rapidly decreases once again to below 2 until the homogeneous hydrolysis starts and the solution is clarified immediately (clarification point, vertically red-dashed line). There is a continued decrease in $\mathrm{pH}$ 
after the homogeneous hydrolysis startes (at $50 \mathrm{~min}$.) to 1.79 at 55 minutes, then an increase back to a level similar to that when the reaction started (2.12) over a 30 minutes period. Such a dramatic drop in $\mathrm{pH}$ is caused by an active oxidization of the combined two catalysts as nitric acid plays the role of the oxidizing agent and acetic acid becomes a target of this reaction as an organic species. This oxidization can occur violently when the acids react with each other at full concentration. In the DCM, however, the TEOS-water mixture provides a diluted environment and the reaction is appropriately controlled, enabling it to take place gradually. The prehydrolyzed sol gradually increases in $\mathrm{pH}$ for another 90 minutes or so to 2.19 , which is virtually equal to the initial $\mathrm{pH}$ of 2.2. The $\mathrm{pH}$ shows continuous increase very slowly for the next 2800 minutes and reaches 2.41 , higher than the initial value, through further observation.

Since the DCM can proceed at room temperature, the temperature change results solely from the enthalpy change of the reaction itself. It was observed that there was a temperature spike of $38^{\circ} \mathrm{C}(311 \mathrm{~K})$ from the room temperature condition $\left(\sim 20^{\circ} \mathrm{C}(293 \mathrm{~K})\right) 5$ minutes after the solution showed immediate clarification, which indicates an active exothermic reaction due to the homogeneous hydrolysis. The temperature spike lasts for less than a minute in this process and it can be prolonged when the reaction proceeds at a large scale. Before and after the temperature spike there is a gradual rise and drop of temperature due to the initiation of the homogeneous hydrolysis and stabilization of the reaction system as it reaches completion of hydrolysis. The temperature of the pre-hydrolyzed sol returns back to the ambient temperature completely 100 minutes after the start of the reaction.

When sulfuric acid was employed instead of nitric acid, another observation was made as shown in Figure 3-4. The overall profiles of the $\mathrm{pH}$ and the temperature changes were recorded similarly with those of the nitric acid - acetic acid combination. The initial $\mathrm{pH}$ (solid triangle on red line) was 0.06 lower (2.14) and the clarification occurred 10 minutes earlier (at 40 minutes, vertically red-dashed line)) when compared to the nitric acid-acetic acid process. Other than 
this, the down trend of $\mathrm{pH}$ and temperature (yellow $\mathrm{X}$ on blue line) after the homogeneous hydrolysis was kicked off, the stabilization time in $\mathrm{pH}$ and temperature (at approximately 120 minutes), and the pot life time (approximately 150 hours), showed great similarity with those of the nitric acid - acetic acid combination, interestingly.

Although these two combinations using the DCM appear similar in terms of changes in key properties, the reaction mechanisms are clearly different as the reaction was driven by differing combinations of catalytic compositions. While the nitric acid - acetic acid combination

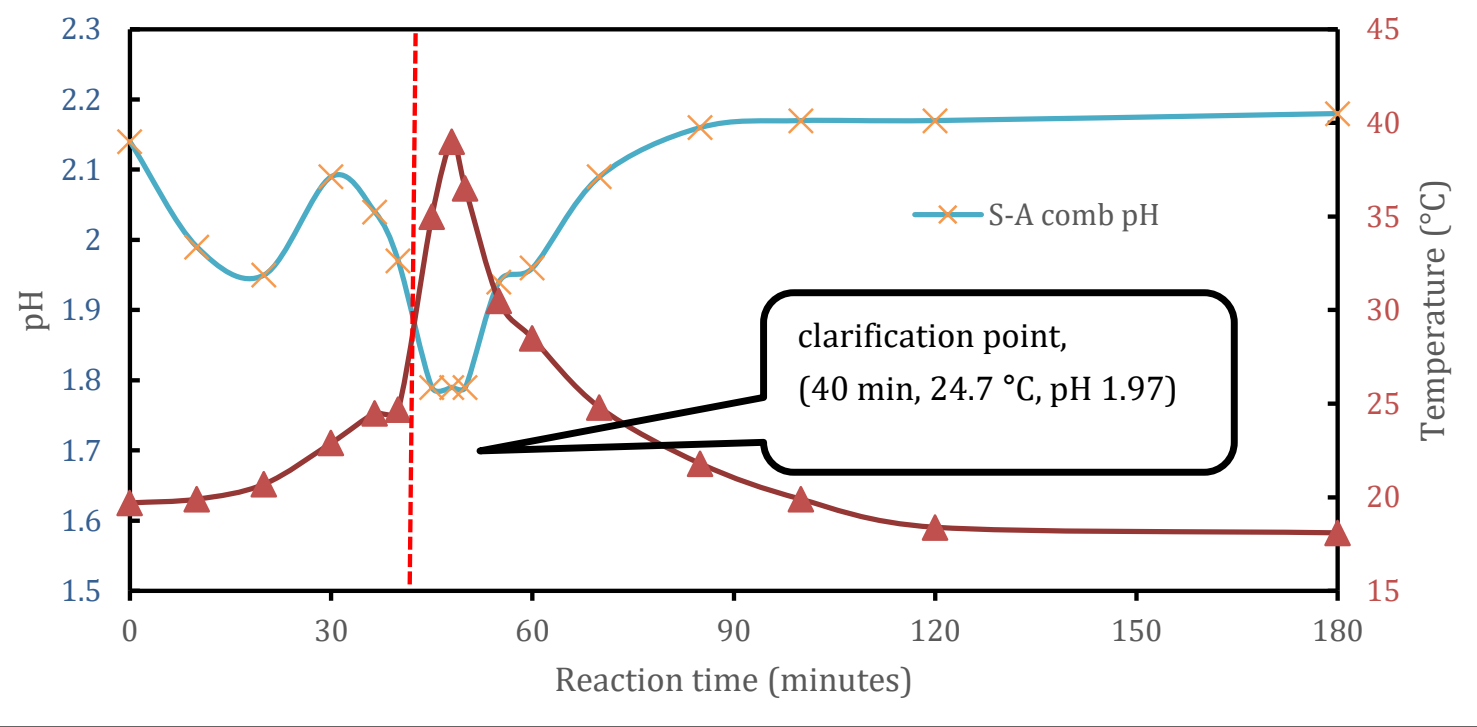

Figure 3-4. pH and temperature profile of the hydrolysis reaction of TEOS by the direct contact method (DCM) when sulfuric acid and acetic acid catalyzed the hydrolysis. The molar ratio of TEOS : water : nitric acid : acetic acid is $1: 4.83: 1.2 \times 10^{-3}: 3.5 \times 10^{-2}$ respectively.

catalyzes the reaction by oxidization as stated earlier, the sulfuric acid - acetic acid combination is worked out as the "superacids" combination first introduced by Hall et al. [110]. Acetic acid 
and sulfuric acid are, respectively, an organic carboxylic acid and an inorganic or "mineral" acid. Oxygen atoms within the carboxyl group $(-\mathrm{COOH})$, though drawn adjacently, are not bound to each other. Actually, the leftmost oxygen atom is bonded only to the carbon atom, forming a carbonyl group $(-C=O)$, while the right oxygen is bonded only to the carbon and hydrogen atoms, $-\mathrm{C}-\mathrm{OH}$. Knowing this, the reaction between the two acids can be described as,

$$
\mathrm{CH}_{3} \mathrm{COOH}+\mathrm{H}_{2} \mathrm{SO}_{4} \rightarrow \mathrm{CH}_{3} \mathrm{C}(\mathrm{OH})_{2}^{+}+\mathrm{HSO}_{4}^{-}
$$

After this reaction, a second molecule of acetic acid is protonated by the remaining bisulfate anion,

$$
\mathrm{CH}_{3} \mathrm{COOH}+\mathrm{HSO}_{4}^{-} \rightarrow \mathrm{CH}_{3} \mathrm{CO}(\mathrm{OH})_{2}+\mathrm{SO}_{4}^{-2}
$$

The above two reactions occur simultaneously. After the first reaction is triggered, superacids result with sufficiently low $\mathrm{pH}$ for the homogeneous hydrolysis to be driven. This reaction, similar to the case of the nitric acid - acetic acid combination, is controlled moderately as the two catalysts are diluted in the TEOS-water mixture.

\subsection{Benefits of the DCM}

A newly developed direct contact method, DCM, that replaces the conventional prehydrolysis step in the two step sol-gel process, enables one to hydrolyze silica alkoxide homogeneously without a diluent and an excessive amount of water, thus producing a clear and pre-hydrolyzed sol. This sol is ready for a further gelation step within a few hours at ambient temperature. The DCM also yields a sol with higher concentration of silica than that obtained from the conventional method so the density range of the final gel product is extended. Such 
Table 3-2. List of characteristic differences between the conventional method and DCM for pre-hydrolysis in silica sol-gel route.

\begin{tabular}{|c|c|c|}
\hline & Conventional Pre-hydrolysis & $\begin{array}{l}\text { Direct Contact Method } \\
\text { (DCM) }\end{array}$ \\
\hline input materials & $\begin{array}{l}\text { precursor, mother liquor, } \\
\text { water, catalyst }\end{array}$ & precursor, water, catalyst \\
\hline hydrolysis completion & in $1.5-2$ hours at $60-65 C$ & $\begin{array}{l}\text { in } 2-3 \text { hours at ambient } \\
\text { temperature }\end{array}$ \\
\hline $\begin{array}{l}\text { Silica content in } \\
\text { pre-hydrolyzed sol }\end{array}$ & 8.6 wt. $\%$ or under & $20.3 w t \%$ or over \\
\hline gelation condition & $\begin{array}{l}\text { diluted and catalyzed with } \\
\text { heat }\end{array}$ & diluted and catalyzed \\
\hline gelation time & $\begin{array}{l}\text { hours to days at elevated } \\
\text { temperature }\end{array}$ & $\begin{array}{l}\text { seconds to hours at } \\
\text { ambient temperature }\end{array}$ \\
\hline $\begin{array}{l}\text { pot life time of } \\
\text { hydrolyzed sol }\end{array}$ & $\begin{array}{l}\text { up to months } \\
\text { (when stored in manufacturer } \\
\text { vacuum sealed container under } \\
\text { refrigeration) }\end{array}$ & $\begin{array}{l}\text { up to a week } \\
\text { (room temperature in an } \\
\text { open container) }\end{array}$ \\
\hline
\end{tabular}

advantages increase the flexibility of the entire sol-gel process with regards to combining with any existing material process.

The produced sols also have pot lifespans that can be as long as a week even when stored at room temperature in an open container. This sufficiently long pot life enables preparation of large quantities of the pre-hydrolyzed active sol, which can then be stored and later used for a variety of different types of gel products as needed. This pot life can be even extended by storing the sol in a refrigerator below $2^{\circ} \mathrm{C}(34 \mathrm{~K})$ under a vacuumed seal, as such conditions can retard the sol-to-gel reaction of this active sol. Finally, the production costs can be remarkably reduced as the time and energy consumed by the DCM is much less compared to the conventional two step process. With these advantages, DCM still produces a pre-hydrolyzed sol that has properties 
compatible to a functional silane such as Dynasilan ${ }^{\circledR}$ SILBOND $^{\circledR}[111]$, which is commercially available at a much higher price than TEOS. Table 3-2 shows a list of the notable differences between conventional pre-hydrolysis and the DCM. 


\section{Chapter 4}

\section{Synthesis and Porosity Study of Silica Aerogel}

A series of monolithic silica aerogels were fabricated via a two step sol-gel process that employees the DCM (direct contact method), aging or rinsing, and the COLD method of $\mathrm{CO}_{2}$ supercritical drying. In this dissertation research, the nitric acid - acetic acid combination in the DCM has been selected as a standard process whose products are characterized and compared to: those produced from different catalytic combinations; those produced with and without the aging step required for hydrophobicity; and those produced with a range of gelation conditions, all with a focus on producing a range of porosities for the ultimate objective of enabling control of thermal conductivity via porosity.

\subsection{Experimental Methods}

Figure 4-1 shows the entire production route used for synthesis of monolithic silica aerogel in this research. This is the standard procedure and includes the various options that can be varied: the catalysts, the aging, and the gelation conditions, all with the goal of observing the change in porosity of the resulting aerogel. 


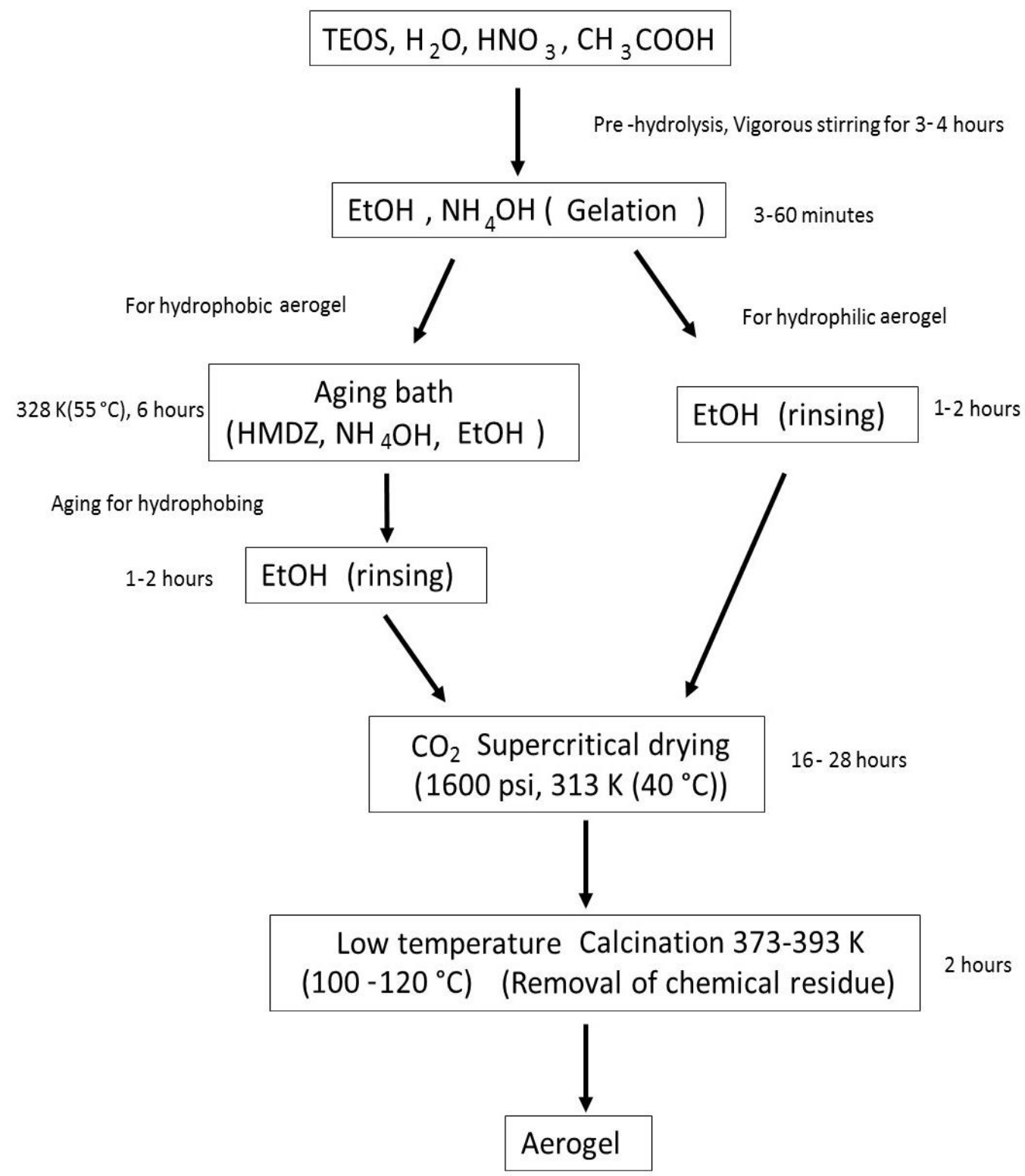

Figure 4-1. Procedure for production of silica aerogel including the DCM process in the sol-gel process by the nitric acid-acetic acid combination ( $\mathrm{N}-\mathrm{A}$ comb.). Also includes the optional route for production of hydrophobic samples. 


\subsubsection{Sol-gel synthesis}

Aerogels were prepared using TEOS (tetraethyl orthosilicate) as the precursor. TEOS was pre-hydrolyzed by the DCM using nitric acid and acetic acid (N-A combination) for the standard samples and sulfuric acid replaces the nitric acid (S-A combination) as the catalyst for the other series of samples. The relative molar ratios of TEOS : water : nitric acid for N-A combination (or sulfuric acid for S-A combination) : acetic acid were $1.0: 4.8: 1.0 \times 10^{-3}\left(1.2 \times 10^{-3}\right): 3.5 x$ $10^{-2}$. The pre-hydrolyzed sol produced from these molar ratios had $20.3 w t \%$ of silica content which is the basis for controlling the target density of the produced gel.

After allowing the hydrolysis reaction to proceed for 3-4 hours, a calculated amount of prehydrolyzed sol was added to an ethanol solution, methanol solution, methanol-ethanol mixture, or ethanol-water mixture with $1.5 \times 10^{-3} \mathrm{vol} \%$ ammonia as a gelation catalyst. This basecatalyzed sol was vigorously stirred and transferred to a disk-shaped mold with an inner diameter of $30 \mathrm{~mm}$. The sol then gelled in the mold within $18 \sim 140$ minutes according to the pre-designed target densities and the structure continued to strengthen for the next 45 to 50 minutes before initiating the aging step.

\subsubsection{Aging - post sol-gel treatment}

This wet gel was then aged in an aging solution prepared by HMDS (1,1,3,3hexamethyldisilazine) diluted in ethanol at $328 \mathrm{~K}\left(55^{\circ} \mathrm{C}\right)$ for 6 hours. This step imparts the hydrophobic behavior on the gel as the methyl groups are attached to the silica chains. The hydrophilic aerogel was prepared by skipping this aging step and directly drying under supercritical conditions. Table 4-1 lists the chemical reagents used for this aerogel process along with the reagent properties. 
Table 4-1. List of reagents used for the silica aerogel production process.

\begin{tabular}{|c|c|c|c|c|c|}
\hline Reagent & Formula & Mol. wt. & $\begin{array}{l}\text { Density } \\
\left(\mathrm{g} / \mathrm{cm}^{3}\right)\end{array}$ & $\begin{array}{l}\text { Boiling Point } \\
\qquad\left({ }^{\circ} \mathrm{C}\right)\end{array}$ & Supplier \\
\hline TEOS & $\mathrm{Si}\left(\mathrm{OC}_{2} \mathrm{H}_{5}\right)_{4}$ & 208.33 & 0.934 & 166 & Aldrich \\
\hline Ethanol & $\mathrm{C}_{2} \mathrm{H}_{5} \mathrm{OH}$ & 46.07 & 0.79 & 78.1 & McMaster \\
\hline Methanol & $\mathrm{CH}_{3} \mathrm{OH}$ & 32.04 & 0.79 & 64.7 & Aldrich \\
\hline $\begin{array}{l}\text { Ammonia } \\
\text { solution }\end{array}$ & $\mathrm{NH}_{4} \mathrm{OH}$ & 40.00 & 0.504 & 36.0 & Cole-Palmer \\
\hline Nitric acid & $\mathrm{HNO}_{3}$ & 63.01 & 1.51 & 83.0 & Fisher \\
\hline Sulfuric acid & $\mathrm{H}_{2} \mathrm{SO}_{4}$ & 98.08 & 1.84 & 337 & Fisher \\
\hline Acetic acid & $\mathrm{C}_{2} \mathrm{H}_{4} \mathrm{O}_{2}$ & 60.05 & 1.049 & $\begin{array}{c}40 \\
\text { (flash point) }\end{array}$ & J.T. Baker \\
\hline HMDS & $\mathrm{C}_{16} \mathrm{H}_{19} \mathrm{NSi}_{2}$ & 161.40 & 0.760 & $\begin{array}{c}20.0 \\
\text { (flash point) }\end{array}$ & Acros \\
\hline
\end{tabular}




\subsubsection{Supercritical drying process and low temperature}

\section{calcination}

The aged wet gel was then rinsed by soaking in successive baths of pure ethanol multiple times to wash away any possible unreacted chemicals, and then finally transformed from a wet gel into an aerogel via the low temperature supercritical drying process in a supercritical extractor unit built as shown in Figure 4-2 (the COLD method). A Polaron critical point dryer (E3100, Polaron) was used as an extractor and an Isotemp 1016D (Fisher Scientific) was used to control the temperature of the extractor conductively.

During the supercritical drying process, solvent exchange occurred and the ethanol liquid was replaced with liquid carbon dioxide at 850 psig and $288 \mathrm{~K}\left(15^{\circ} \mathrm{C}\right)$. When the fluid exchange

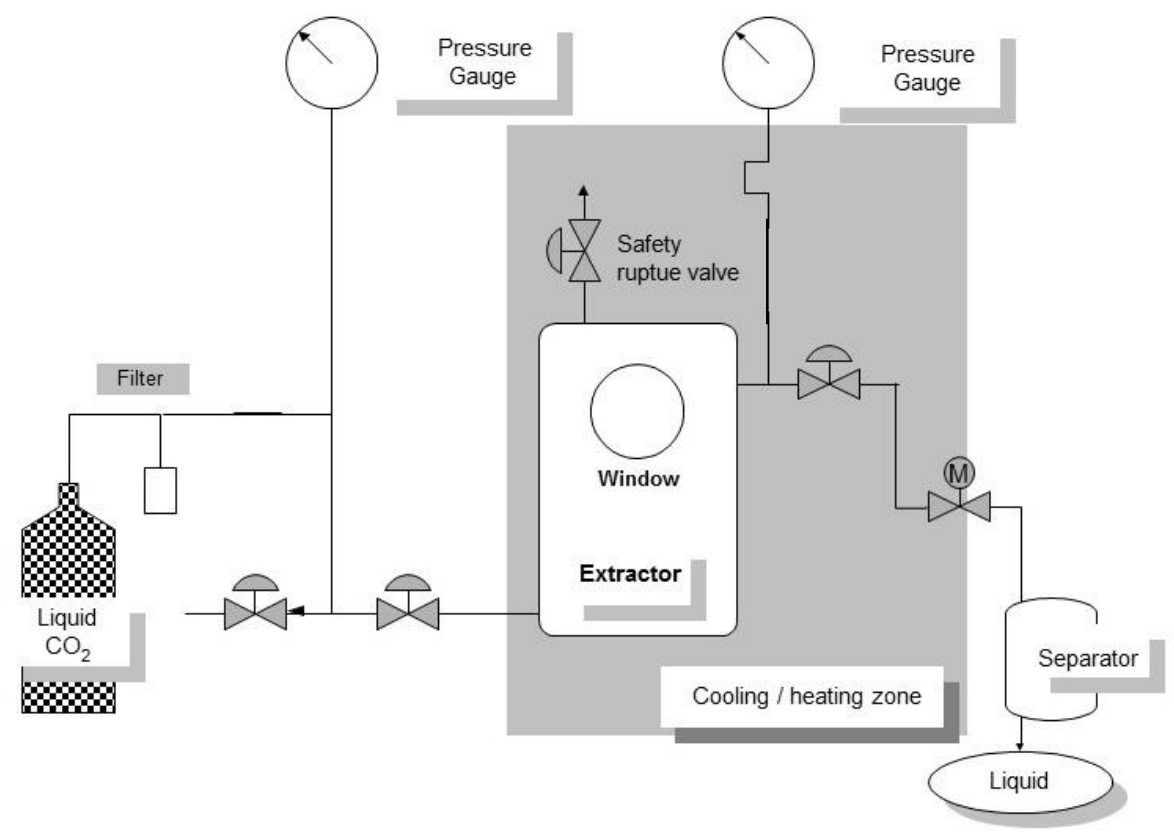

Figure 4-2. Schematic diagram of the supercritical drying unit for the COLD method. 
step was completed, the drying system was heated to $313 \mathrm{~K}\left(40^{\circ} \mathrm{C}\right)$ and the pressure increased to 1600 psig, transforming the liquid $\mathrm{CO}_{2}$ inside the autoclave to a supercritical fluid. Figure 4-3

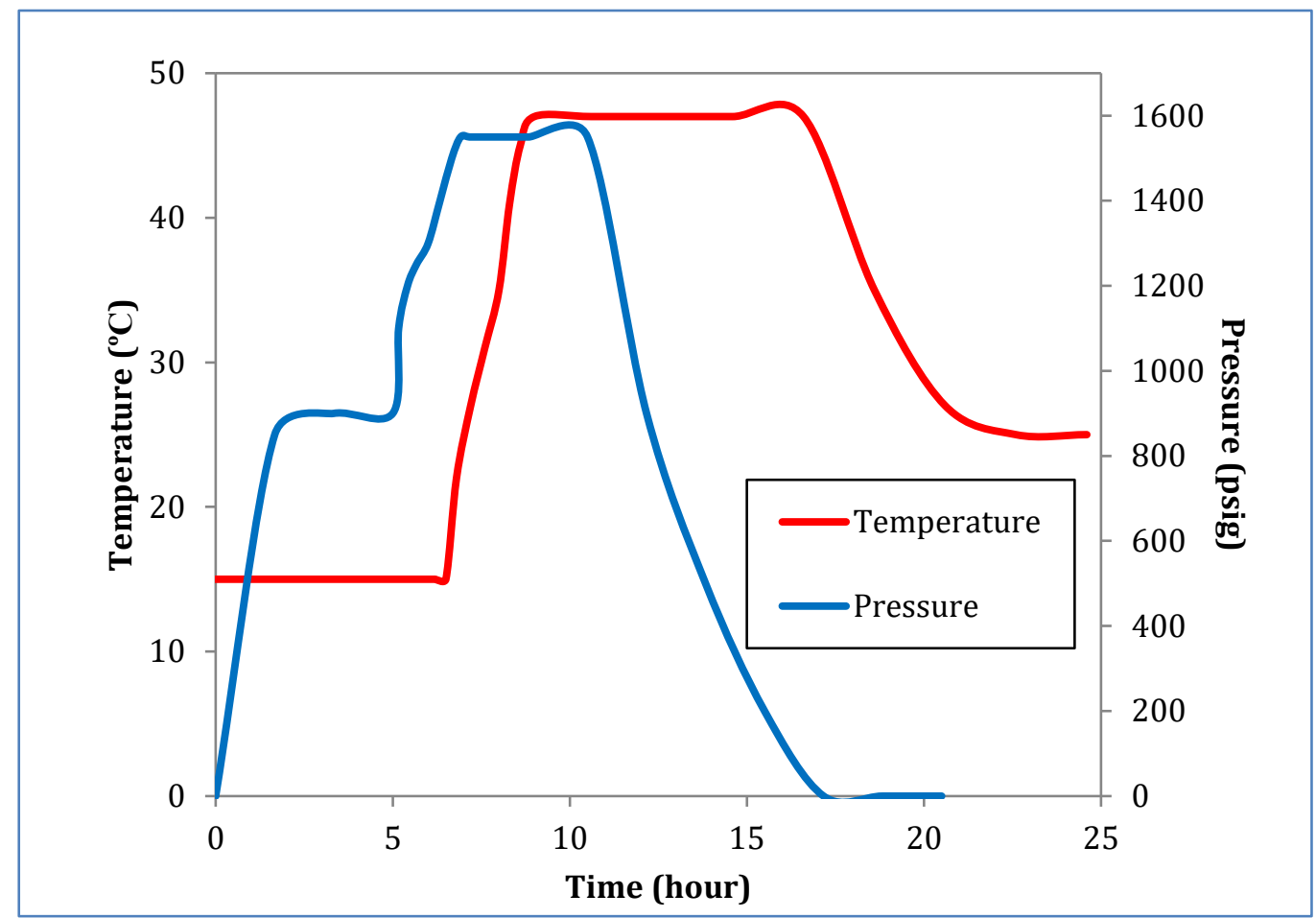

Figure 4-3. Temperature and pressure profiles of $\mathrm{CO}_{2}$ supercritical drying process (the COLD method).

shows the temperature-pressure changes while the $\mathrm{CO}_{2}$ changes from a liquid to the supercritical phase, and back to the gaseous phase for the depressurization process. Once the supercritical $\mathrm{CO}_{2}$ is vented from the autoclave, the air-filled silica aerogel remains. When unloaded from the autoclave this as-dried aerogel has chemical residue due to the byproducts of the sol-gel reaction and excessive water molecules. These impurities can be removed by low temperature calcination. The aerogel was calcinated at $373 \sim 393 \mathrm{~K}\left(100 \sim 120^{\circ} \mathrm{C}\right)$ at a heating rate of $2^{\circ} \mathrm{C} / \mathrm{min}$ for 2 hours in a convection oven. The low temperature calcination enabled evaporation of any residual chemicals while the inherent structure of the aerogel remained intact. 


\subsection{Characterization}

Structural properties of the produced aerogels were characterized mainly by nitrogen adsorption-desorption with the porosity estimated by measuring the apparent density and skeletal density. Brunauer-Emmett-Teller (BET) surface areas, the Barrett-Joyner-Halenda $(\mathrm{BJH})$ pore volumes, averaged pore diameter, and pore size distributions were measured by nitrogen adsorption-desorption at $77 \mathrm{~K}\left(-196^{\circ} \mathrm{C}\right)$ using a Micromeritics ASAP 2010 instrument $(0.073 \%$ of uncertainty in reading measured properties of a sample) [112]. Both methods have been well recognized as trustworthy methods to characterize surface area and the porous structure of aerogel over decades. There were more than 800 articles since 2000 including BET and/or BJH characterization of aerogel, and these works all contribute to further understanding of aerogel's properties from its unique structure. Results of these techniques are accepted with assumptions that nitrogen gas molecules perfectly occupy the pores in aerogel and there is no physical and chemical interaction between the nitrogen molecules.

The instrument uses the BET method to evaluate the specific surface area of a material in $\mathrm{m}^{2} / \mathrm{g}$ using nitrogen multilayer adsorption measured as a function of relative pressure. For each data point for surface area calculation, the BET transformation is calculated as follows,

$$
B_{I}=\frac{P_{r e l}}{\left(1-P_{r e l}\right) \times N_{a d s}}
$$

where $\mathrm{B}_{\mathrm{I}}$ is the dependable variable such as slope $(S)$ and $\mathrm{Y}$-intercept $\left(Y_{\text {int }}\right), \mathrm{P}_{\text {rel }}$ is the relative pressure, and $N_{a d s}$ is the amount of nitrogen gas molecule after equilibrium [113]. After multiple data points are acquired, a least-square fit is performed on the designated pairs $\left(P_{r e l}, B_{I}\right)$ and $S$ and $Y_{\text {int }}$ are calculated. Using the results of these calculations, the following BET surface area can be calculated, 


$$
\mathrm{SA}_{\mathrm{BET}}=\frac{\text { cross }- \text { sectional area of } N_{2} \text { gas } \times(6.023 \times 1023)}{C c \times\left(S+Y_{\text {int }}\right)}
$$

where $\mathrm{Cc}$ is the correlation coefficient determined by measuring a reference sample during the instrument calibration [113].

The mesopore size distributions were calculated applying the BJH method to the desorption branch of the isotherm. This method is a procedure for calculating pore size distribution from experimental isotherms using the Kelvin model of pore filling,

$$
\ln \left(\frac{P_{C}}{P_{o}}\right)=\frac{-2 \gamma}{R T \rho H}
$$

where $P_{c}$ is the pressure at which pore of width $H, P_{o}$ is the saturation pressure of the bulk fluid, $R$ is the ideal gas constant, and $\gamma$ and $\rho$ are the surface tension of nitrogen at absolute temperature and the liquid density [114]. It applies to the mesopore size of pores so the BJH method can profiles the pore size distribution of aerogel effectively as its main peak is in a range of mesopore size. Prior to the measurement, all samples were outgassed at $383 \mathrm{~K}\left(110^{\circ} \mathrm{C}\right)$ for 12 hours. In

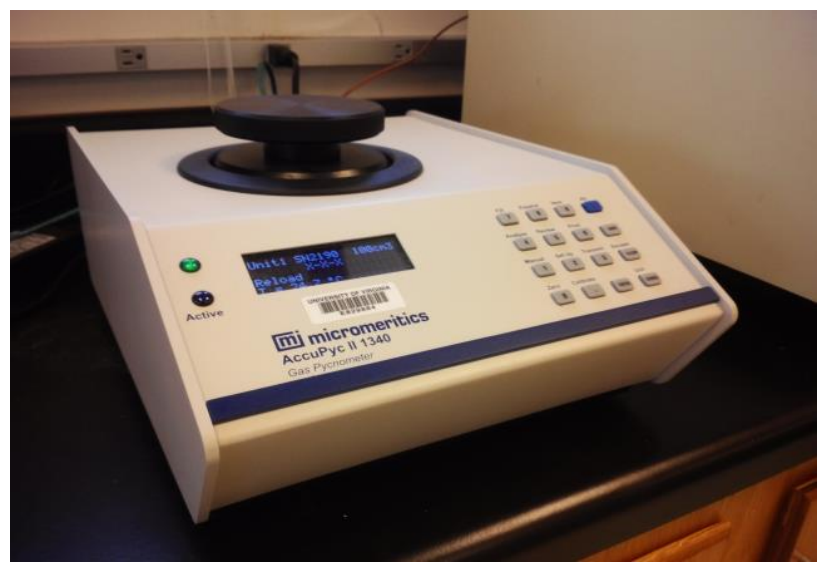

Figure 4-4. AccuPyc II 1340 gas pycnometer at the Aerogel Research Lab (AeRL). 
addition, scanning electron microscopy (SEM) and morphological comparison were selectively used for further understanding of the textural properties of the aerogel related to the porosity.

As introduced in Chapter 2, the skeletal densities of the produced aerogels were measured using a helium pycnometer, an AccuPyc II 1340 (Figure 4-4, Micromeritics). This is a fully automated gas displacement pycnometer which determines skeletal density and volume by measuring the pressure change of helium within a calibrated volume. Once the sample is loaded, the pycnometer runs ten cycles of helium gas charging and discharging gas steps, and averages the results from these multiple runs in order to increase the accuracy of the measured value. The device has an uncertainty of $0.06 \%$ and reproducibility guaranteed to within $0.02 \%$ according to equipment specifications.

The bulk density (apparent density) of aerogel is simply the ratio of mass to volume of the monolithic sample. Mass was measured with a scale with an accuracy of $\pm 0.04 \mathrm{mg}$ and the dimensions of the aerogel were measured with an accuracy of $\pm 0.0005 \mathrm{~mm}$. Thus, the overall uncertainty in the apparent (bulk) density is $\pm 0.00019 \mathrm{~g} / \mathrm{cm}^{3}$.

\subsection{Sample Preparations}

To enable exploration of the effects of various processing parameters on the resulting porosity and texture of the aerogel, a variety of production modifications were explored as summarized below. 


\subsubsection{Variations in target density and porosity of "standard}

\section{samples" via gelation time}

As previously described the "standard" processing condition explored in this dissertation uses TEOS (tetraethyl orthosilicate) as the precursor, pre-hydrolyzed using nitric acid and acetic acid (N-A comb), in the DCM process. Nine standard samples were thus prepared, adjusting

the target density from 0.0194 to $0.1358 \mathrm{~g} / \mathrm{cm}^{3}$ in intervals of approximately $0.01-0.02$ $\mathrm{g} / \mathrm{cm}^{3}$. The target density was controlled by considering the silica content $(20.3 \mathrm{wt} \%$, see Section 4.1.1) in the whole volume of the gel that includes the pre-hydrolyzed sol and the solvent. After estimating the amount of silica solid for a specific target density, the quantified amount of prehydrolyzed sol that had the corresponding amount of silica solid was utilized in the gelation process.

In the gelation, this sol was mixed with the solvent that had also been quantified and mixed with the gelation catalyst, so the total volume of the pre-hydrolyzed sol and the solvent satisfied the volumetric condition of the planned target density. The gelation catalyst was only $0.15 \mathrm{vol} \%$ so it was neglected in the target density calculation. These nine samples were used for a basic investigation of porosity and structural properties.

An additional three samples were also prepared by locating additional target densities in the middle-low, the middle, and the high values of the range studied $\left(0.0194-0.1358 \mathrm{~g} / \mathrm{cm}^{3}\right)$. These three samples were expected to allow a more detailed examination of the relationship between target density and gelation time in "standard samples". Thus, a total of twelve standard samples were used to compare the influence of target density on gelation time as the gelation time is one of the most important processing conditions in the production environment. The 
specifics of these twelve standard samples (the quantified amount of pre-hydrolyzed sol, the amount of solvent, and the target density) along with their Sample ID are given in Table 4-2 .

Table 4-2. List of samples of monolithic silica aerogels and their processing conditions prepared by the standard process (the nitric acid - acetic acid combination for the DCM catalyst). The three additional samples for the gelation time investigation are 2.0/8.0, 3.5/6.5, and 7.0/3.0 (highlighted in yellow).

\begin{tabular}{lccc}
\hline \hline Sample ID & $\begin{array}{c}\text { pre-hydrolyzed sol } \\
(\mathbf{m l})\end{array}$ & $\begin{array}{c}\text { diluent (ethanol) } \\
(\mathbf{m l})\end{array}$ & $\begin{array}{c}\text { target density } \\
\left(\mathbf{g} / \mathbf{c m}^{3}\right)\end{array}$ \\
\hline $1.0 / 9.0$ & 1.0 & 9.0 & 0.0194 \\
$1.5 / 8.5$ & 1.5 & 8.5 & 0.0291 \\
\hline $2.0 / 8.0$ & 2.0 & 8.0 & 0.0388 \\
$2.5 / 7.5$ & 2.5 & 7.5 & 0.0485 \\
$3.0 / 7.0$ & 3.0 & 7.0 & 0.0582 \\
$3.5 / 6.5$ & 3.5 & 6.5 & 0.0679 \\
$4.0 / 6.0$ & 4.0 & 6.0 & 0.0776 \\
$4.5 / 5.5$ & 4.5 & 5.5 & 0.0873 \\
$5.0 / 5.0$ & 5.0 & & 0.0970 \\
$6.0 / 4.0$ & 5.0 & 5.0 & 0.1164 \\
$6.6 / 3.4$ & 6.0 & 3.0 & 0.1213 \\
$7.0 / 3.0$ & & & \\
\hline \hline
\end{tabular}




\subsubsection{Variations in solvent types of "standard samples" via changing gelation time}

In order to further study the effects of solvent type on porosity and aerogel structure, the solvent type was changed in three ways. First, the ethanol, which is the solvent of the standard process, was replaced with methanol to observe how the nature of the solvent affects porosity and the formation of the aerogel structure. Second, a methanol-ethanol mixture was used as the solvent.

Table 4-3. List of samples of monolithic silica aerogels prepared by different solvent type. All samples were produced at the target density of $0.0582 \mathrm{~g} / \mathrm{cm}^{3} \mathrm{so} 3.0 \mathrm{ml} \mathrm{of}$ the pre-hydrolyzed sol produced by the standard DCM was used to prepare each samples.

Solvent (diluent) type

$3.0 / 7.0$

$3.0 / 7.0 \mathrm{M}$

3.0/7.0ME

$6 \mathrm{Et} / 1 \mathrm{~W}$

$5 E t / 2 W$
$7.0 \mathrm{ml}$ ethanol

$7.0 \mathrm{ml}$ methanol

$3.5 \mathrm{ml}$ ethanol and $3.5 \mathrm{ml}$ methanol

$6.0 \mathrm{ml}$ ethanol and $1.0 \mathrm{ml}$ (14 vol\%) water

$5.0 \mathrm{ml}$ ethanol and $2.0 \mathrm{ml}$ ( $28 \mathrm{vol} \%$ ) water 
These two alcohols were mixed at equal volumetric ratio (50 vol\%) and added to the prehydrolyzed sol as the solvent during gelation. In the standard process, ethanol was selected because it is a mother liquor of TEOS after the sol-gel reaction is complete. Use of mother liquor as a solvent prevents trans esterification, which is a reverse reaction of the hydrolysis in the solgel process. On the other hand, non-mother liquor can be employed to intentionally reduce the

Table 4-4. List of samples of monolithic silica aerogels and their processing conditions produced by different catalytic combination of sulfuric acid - acetic acid for the DCM.

\begin{tabular}{|c|c|c|c|}
\hline Sample ID & $\begin{array}{l}\text { pre-hydrolyzed sol } \\
\qquad(\mathrm{ml})\end{array}$ & $\begin{array}{c}\text { diluent (ethanol) } \\
(\mathrm{ml})\end{array}$ & $\begin{array}{c}\text { target density } \\
\left(\mathrm{g} / \mathrm{cm}^{3}\right)\end{array}$ \\
\hline SA1.0/9.0 & 1.0 & 9.0 & 0.0194 \\
\hline SA1.5/8.5 & 1.5 & 8.5 & 0.0291 \\
\hline SA2.0/8.0 & 2.0 & 8.0 & 0.0388 \\
\hline SA2.5/7.5 & 2.5 & 7.5 & 0.0485 \\
\hline SA3.0/7.0 & 3.0 & 7.0 & 0.0582 \\
\hline SA4.0/6.0 & 4.0 & 6.0 & 0.0776 \\
\hline SA5.0/5.0 & 5.0 & 5.0 & 0.0970 \\
\hline SA6.0/4.0 & 6.0 & 4.0 & 0.1164 \\
\hline SA7.0/3.0 & 7.0 & 3.0 & 0.1358 \\
\hline
\end{tabular}


hydrolysis rate. The slow hydrolysis rate retards the subsequent poly condensation rate of the gelation. This slow gelation enables formation of highly uniform pore sizes by slow crosslinking of the silica chains $[8,15]$. The result from use of the mixed solvent was expected to show how porosity and gel structure are affected by the co-existence of the mother liquor and non-mother liquor as gelation occurs. Third, different ethanol-water mixtures were tested as the solvent.

Ethanol was diluted with water at two different concentrations of 14 and 28 vol\% respectively, so the impact of the existence of the water in the solvent on the porosity and the gel structure could be explored. Table 4-3 lists the details on the samples used to investigate the influence of differences in the solvent type applied for this study.

\subsubsection{Variations in the catalytic combination in the DCM}

As illustrated in Table 4-4, the effects of the catalytic combination in the DCM were also studied by using sulfuric acid - acetic acid combination. Nine samples were produced with target densities in the same range as the standard samples produced $\left(0.0194\right.$ to $\left.0.1358 \mathrm{~g} / \mathrm{cm}^{3}\right)$ in Section 4.3.1. Gelation times were recorded for processes utilizing the same amount of the gelation catalyst as used for the standard samples and the times were compared. One of the nine samples was also selected and characterized to observe differences in the BET surface area, pore size distribution, and porosity from those of the standard sample produced with the same target density as this selected one. 


\subsubsection{Variations in aging process}

The last change in the processing conditions investigated was made to the aging process. As the standard process produces hydrophilic silica aerogel, the aging process was changed into a post sol-gel process in order to produce hydrophobic aerogel. During this post processing step, alkyl groups are attached to the silica chains that create hydrophobicity. This chemical change was expected to affect the structural properties, including porosity and pore size distribution, so three hydrophobic silica aerogels were repeatedly produced at a specific target density and gelation time, apparent density, and porosities were averaged to compare to those of the standard sample produced at the same target density. The BET surface area and pore size distribution were obtained from one of the three samples and also compared to those of the standard sample. Table 4-5 lists the samples produced by different aging processes.

Table 4-5. List of samples of hydrophilic and hydrophobic monolithic silica aerogels and their aging process. Samples were produced with a target density of 0.0582 $\mathrm{g} / \mathrm{cm}^{3}$ so $3.0 \mathrm{ml}$ of the pre-hydrolyzed sol produced by the standard DCM was used to prepare each sample.

\begin{tabular}{lcc}
\hline \hline sample ID & $\begin{array}{c}\text { sample property } \\
\left(\text { target density : } 0.0582 \mathrm{~g} / \mathrm{cm}^{3}\right)\end{array}$ & aging process \\
\hline $3.0 / 7.0$ & Hydrophilic aerogel & Rinsed in ethanol for 1-2 hours \\
HB3.0/7.0 & Hydrophobic aerogel & $\begin{array}{r}\text { Aged in HMD (1,1,3,3- } \\
\text { hexamethyldisilazine) for } 6 \text { hours at 550C } \\
\text { followed by ethanol rinse for } 1-2 \text { hours }\end{array}$ \\
\hline
\end{tabular}




\subsection{Results and Discussion}

\subsubsection{Effects of target density on gelation time of monolithic}

\section{silica aerogel}

Gelation time in the two step sol-gel synthesis is affected by the synthetic conditions which include silica content, amount and type of catalyst, aging of the pre-hydrolyzed sol, and nature of the diluent. Having all other parameters unchanged, different target densities were applied for each sample and gelation was observed by the tilt method [115]. The "tilt method" is a simple technique used to confirm the completion of gelation. The disk mold containing the sol-to-gel solution is tilted and observed to ensure there is no movement of the solution.

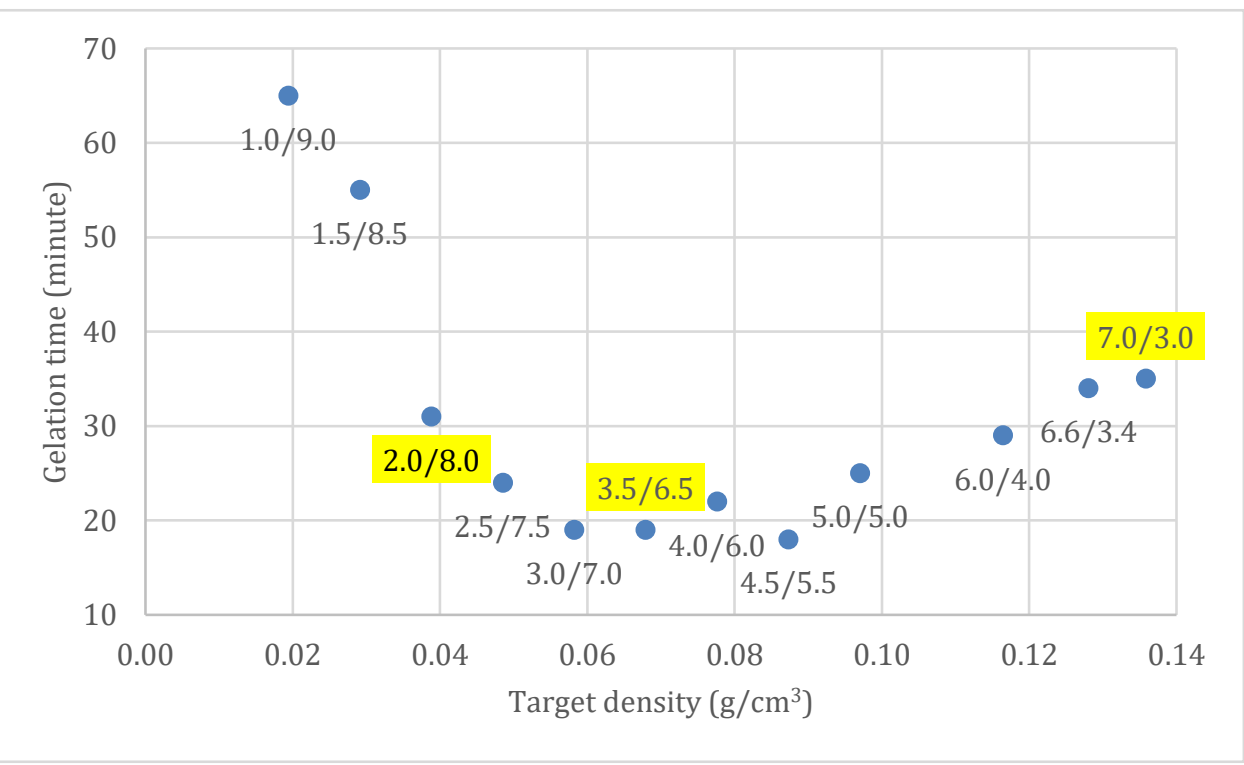

Figure 4-5. Effects of target density on gelation time for the monolithic silica aerogel produced by nitric acid - acetic acid combination for the DCM during sol-gel synthesis (standard process). The three additional samples for the gelation time investigation are 2.0/8.0,3.5/6.5, and 7.0/3.0 (highlighted in yellow). The uncertainties are $\pm 5 \%$ and $\pm 2 \%$ in target density and gelation time, respectively. 
When the nitric acid - acetic acid combination was used in the DCM (the standard process), the gelation time decreased as the target density increased up to near $0.0582 \mathrm{~g} / \mathrm{cm}^{3}(3.0 / 7.0)$, and it stayed at nearly this same level until the target density became approximately $0.0970 \mathrm{~g} / \mathrm{cm}^{3}$

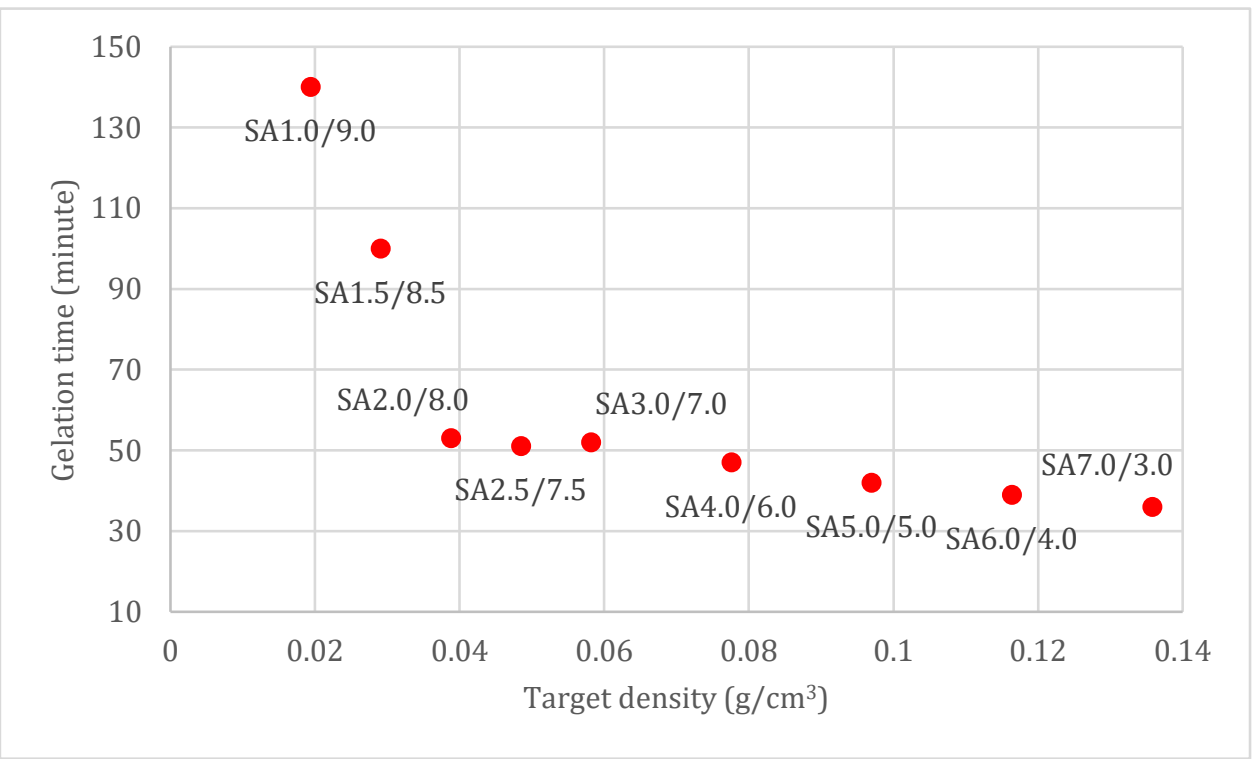

Figure 4-6. Effects of target density on gelation time for the monolithic silica aerogel produced by sulfuric acid - acetic acid combination for the DCM during the sol-gel synthesis. The uncertainties are $\pm 5 \%$ and $\pm 2 \%$ in target density and gelation time respectively.

(5.0/5.0). The gelation took considerably longer when the target density was larger than 0.0970 $\mathrm{g} / \mathrm{cm}^{3}$ as shown in Figure 4-5. Such change of pattern in the gelation time occurs because the increasing silanol groups that must be catalyzed for poly condensation slow down the overall gelation reaction as the target density increases. 
Figure 4-6 shows the gelation time pattern as the target densities were changed when the sulfuric acid - acetic acid combination was employed in the DCM. Notably different than the standard process, the gelation time was steadily and slowly reduced after a sharp drop between $0.0194 \mathrm{~g} / \mathrm{cm}^{3}(\mathrm{SA} 1.0 / 9.0)$ and $0.0388 \mathrm{~g} / \mathrm{cm}^{3}(\mathrm{SA} 2.0 / 8.0)$ as the target density was increased up to $0.1358 \mathrm{~g} / \mathrm{cm}^{3}$ (SA7.0/3.0). The overall gelation times were greater compared to those of the standard samples, which were produced from the DCM with the nitric acid - acetic acid combination.

\subsubsection{Effects of solvent types on gelation time of monolithic}

\section{silica aerogel}

Table 4-6. Effects of solvent types on gelation time of monolithic silica aerogel.

\begin{tabular}{lccccc}
\hline \hline sample ID & $3.0 / 7.0$ & $3.0 / 7.0 \mathrm{M}$ & $3.0 / 7.0 \mathrm{ME}$ & $6 \mathrm{Et} / 1 \mathrm{~W}$ & $5 \mathrm{Et} / 2 \mathrm{~W}$ \\
\hline \hline $\begin{array}{l}\text { diluent } \\
\text { composition }\end{array}$ & ethanol & methanol & $\begin{array}{c}\text { ethanol/methanol } \\
(50 \% / 50 \%)\end{array}$ & $\begin{array}{c}\text { ethanol/water } \\
(86 \% / 14 \%)\end{array}$ & $\begin{array}{c}\text { ethanol/water } \\
(72 \% / 28 \%)\end{array}$ \\
$\begin{array}{l}\text { gelation time } \\
\text { (minutes) } \\
\begin{array}{l}\text { (Uncertainty: } \\
\pm 5 \%)\end{array}\end{array}$ & 19 & 82 & 53 & 58 & 93 \\
\hline \hline
\end{tabular}

Table 4-6 shows the gelation times observed for the various solvent types investigated in the production of monolithic silica aerogel. Based on the processing condition for the 
standard sample with a target density of $0.0582 \mathrm{~g} / \mathrm{cm}^{3}$, it is observed that the gelation time increases when water or methanol are used partially and wholly as diluents. This result suggests that the poly condensation is affected when different solvents from the mother liquor of the precursor (it is ethanol for TEOS) or any additives (water in this research) are mixed even with the mother liquor as a diluent because it hinders the silanol groups from chaining with each other to form a silica network by occupying the space where the condensation occurs continuously. Consequently, the gelation is retarded and the time taken for the formed silica networks to be strengthened increases as observed in the gelation time changes.

\subsubsection{Effects of apparent density on transparency of}

\section{monolithic silica aerogel}

The produced silica aerogel monoliths have good transparency over some target density range, indicating their suitability for insulation for dual pane window systems [116]. Figure 4-7 displays the change in transparency of monolithic disc type silica aerogels produced using the

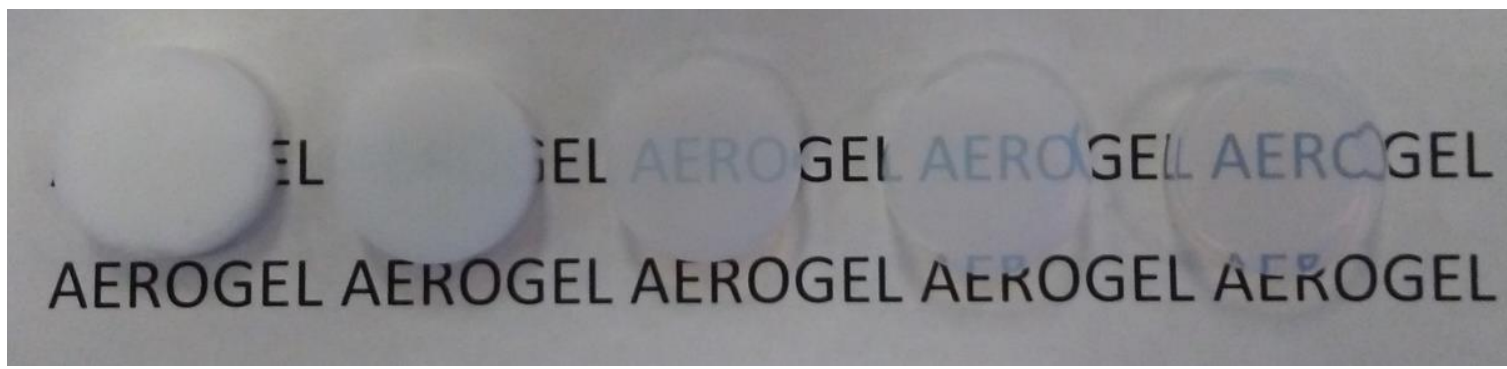

Figure 4-7. Transparency comparison of monolithic silica aerogel based on the apparent density (prepared by the standard process). The apparent densities of each aerogel from the left are $0.0529,0.1244,0.1285,0.1733$, and $0.2188 \mathrm{~g} / \mathrm{cm}^{3}$, respectively. The sample IDs from the left are 1.5/8.5, 2.5/7.5, 3.0/7.0, 4.0/6.0, and 5.0/5.0. 
DCM method with nitric acid - acetic acid combination with different apparent (actual, or bulk) densities. As clearly seen in the picture, the transparency is increased with higher apparent density that results from a higher target density in general. This is quite different from that

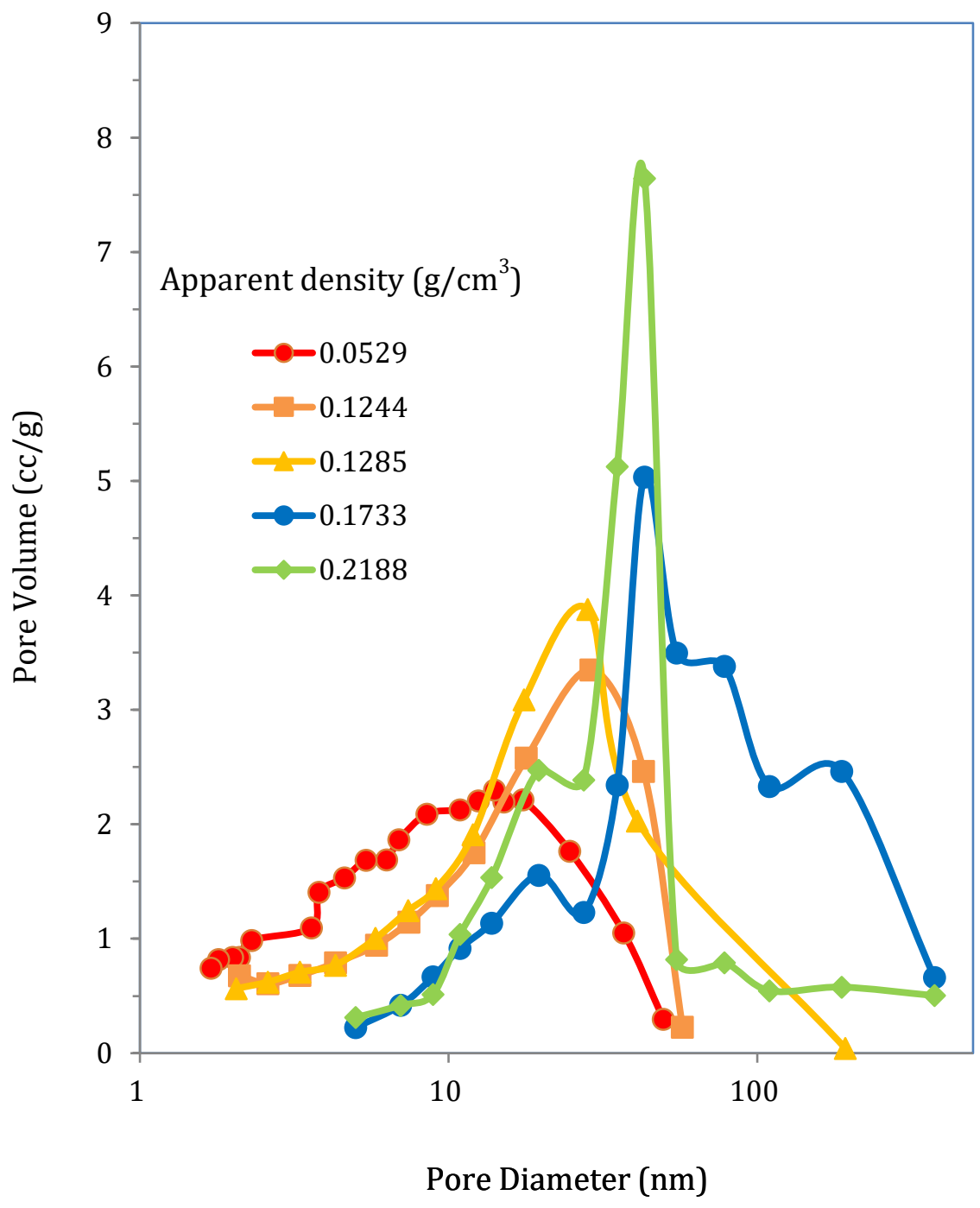

Figure 4-8. Effects of apparent density $(\mathrm{g} / \mathrm{cm} 3)$ on pore size distributions of monolithic silica aerogels prepared by the standard process (the nitric acid - acetic acid combination for the DCM). The sample IDs from the lowest apparent density are 1.5/8.5, 2.5/7.5, 3.0/7.0, 4.0/6.0, and 5.0/5.0, respectively. 
expected with regard to the morphological properties, as the lower target density may result in thinner silica backbones with more spaces (pores) between them which appear to scatter less light, hence the corresponding sample aerogel possesses greater transparency.

However, this unexpected result was explained when the pore size distributions of the sample aerogels from the BJH method (nitrogen desorption) were compared for the different apparent densities as illustrated in Figure 4-8. It was observed that a lower apparent density results from the lower target density, which causes the produced monolithic silica aerogel to have a broader range of pore size distributions. The width of the pore size distribution narrows as the apparent density is increased with higher target density. While the three-dimensional thickness of the silica backbones of the aerogel can affect the transparency of the silica aerogel as mentioned earlier, such narrower pore size distributions can also be considered as another textural property that influences the transparency of the monolith. It turns out that the latter property in this performed synthetic method influences the light-transmitting capacity over the former one as the structural parameter which results in more transparent silica aerogel. Furthermore, the silica backbones of the aerogel with lower target density can be thicker than those of the aerogel with higher target density, which will be discussed more in the next section (4.4.4). Therefore, both the thicker silica backbones and the less homogeneous pore size distribution observed in the lower density aerogels, which indicates a more three-dimensionally crosslinked microstructure, lead to scattering of visible light, thus yielding translucent or opaque silica aerogels. It is also observed that physically, the lower density aerogel has a bit more elasticity. This behavior is believed to be caused by the locally entangled gel structures, which are similar to those observed typically in low-density polymers. As the SEM image (Figure 13) shows, the sizes of the entangled structures can be several hundred nanometer in diameter, this will be discussed more in Section 4.4.6. These coagulated solid parts are large enough to scatter visible light, which has a spectral range of $380 \sim 780 \mathrm{~nm}$. 
Table 4-7. Effects of target density on textural properties of monolithic silica aerogels.

\begin{tabular}{lcccc}
\hline \hline sample ID & $\begin{array}{c}\text { target density } \\
\left(\mathrm{g} / \mathrm{cm}^{3}\right)\end{array}$ & $\begin{array}{c}\text { BET surface area } \\
\left(\mathrm{m}^{2} / \mathrm{g}\right)\end{array}$ & $\begin{array}{c}\text { pore volume } \\
\left(\mathrm{N}_{2} \text { desorption }\right) \\
(\mathrm{cc} / \mathrm{g})\end{array}$ & $\begin{array}{c}\text { average } \\
\text { pore diameter } \\
(\mathrm{nm})\end{array}$ \\
\hline $1.5 / 8.5$ & 0.0291 & 1008.3 & 2.21 & 7.49 \\
$2.5 / 7.5$ & 0.0485 & 936.5 & 2.48 & 10.43 \\
$3.0 / 7.0$ & 0.0582 & 955.2 & 2.63 & 10.33 \\
$4.0 / 6.0$ & 0.0776 & 1036.8 & & 12.24 \\
$5.0 / 5.0$ & 0.0970 & 1169.2 & 3.17 & \\
& & & 3.17 & \\
\hline \hline
\end{tabular}

Table 4-7 lists BET surface areas, pore volumes, and average pore diameters of the silica aerogels analyzed and discussed above with the range of target densities. We can see an increase of the pore volume with increase of the target density while the surface areas show unclear differences but remain around $1000 \mathrm{~m}^{2} / \mathrm{g}$, with the exception of the one with the highest target density $\left(0.097 \mathrm{~g} / \mathrm{cm}^{3}\right)$, which has a surface area above $1100 \mathrm{~m}^{2} / \mathrm{g}$. The average pore diameters in this studied range showed increases (from $7.49 \mathrm{~nm}$ to $12.24 \mathrm{~nm}$ ) with increased target density from $0.029 \mathrm{~g} / \mathrm{cm}^{3}$ up to $0.077 \mathrm{~g} / \mathrm{cm}^{3}$, but then a decrease to $10.94 \mathrm{~nm}$ at the highest target density, $0.097 \mathrm{~g} / \mathrm{cm}^{3}$. These independent trends between the textural properties discussed show that the silica aerogel production process can be adjusted to optimize a selected property among them, including the porosity of silica aerogel that will be discussed later. 


\subsubsection{Effects of target density on apparent density and skeletal density}

When silica aerogel is synthesized, the actual apparent density is higher than the target density, which is assumed to be identical to the final density in theory, mainly due to synerisis effects during the aging and rinsing period before the wet gel is supercritically dried. There is

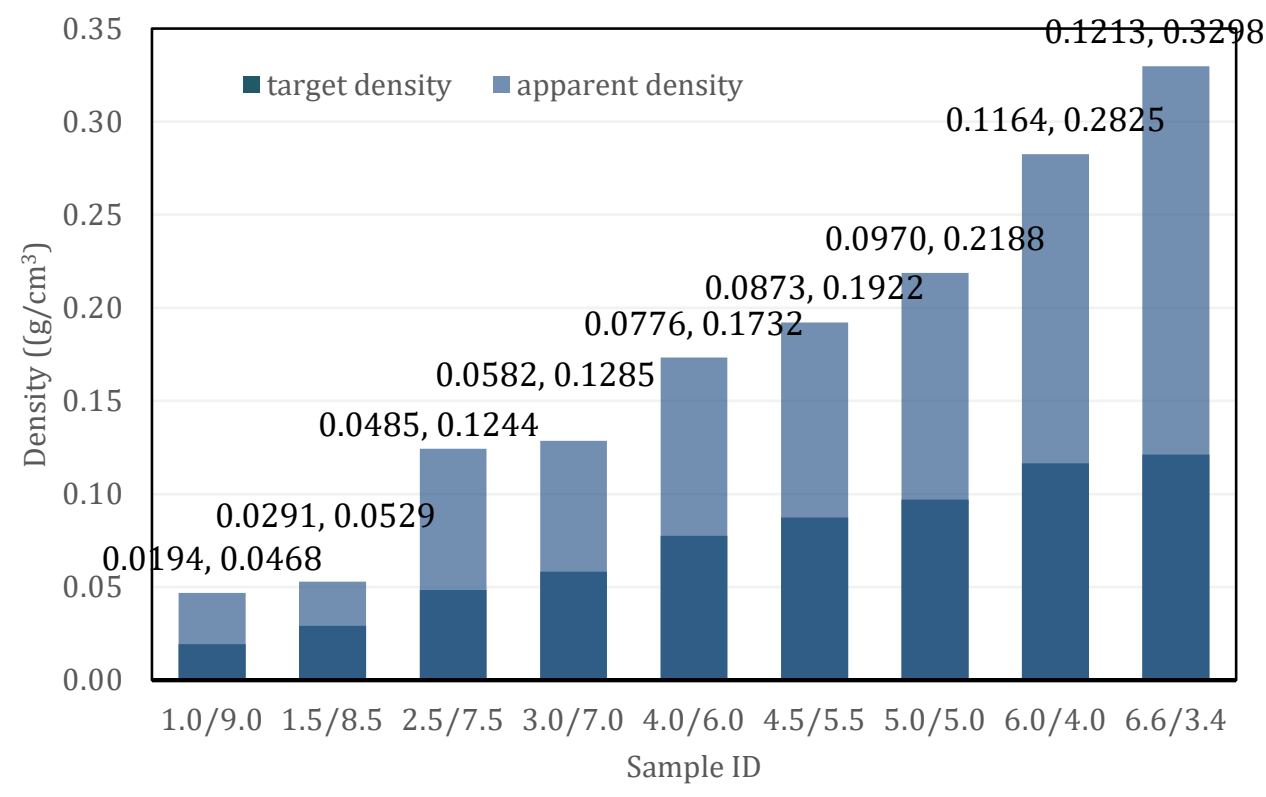

Figure 4-9. Effects of target density on actual apparent density of monolithic silica aerogel prepared by the standard process (the nitric acid - acetic acid combination for the DCM). The left value of the data label is the target density $\left(\mathrm{g} / \mathrm{cm}^{3}\right)$ and the right one is the apparent density $\left(\mathrm{g} / \mathrm{cm}^{3}\right)$ for each sample. The uncertainties are \pm $5 \%$ and $\pm 0.19 \%$ in target density and apparent density, respectively. 
also minor collapsing of the inherent pores of the alcogel during supercritical drying due to incomplete solvent removal with liquid $\mathrm{CO}_{2}$. Figure 4-9 shows the differences between these two densities as the range of the target density of the produced silica aerogels increases from 0.0194 (sample ID:1.0/9.0) to $0.1213 \mathrm{~g} / \mathrm{cm}^{3}$ (sample ID:6.6/3.4). With the variation of \pm 0.4507 $\mathrm{g} / \mathrm{cm}^{3}$, the average ratio of the apparent density to the target density is 2.31 .

Interestingly, the skeletal density shows a remarkably different trend when compared to the target density as shown in Figure 4-10. Generally, the skeletal density descreases with increasing target density. The highest value of skeletal density $\left(2.2150 \mathrm{~g} / \mathrm{cm}^{3}\right)$ was observed for a target density of $0.0194 \mathrm{~g} / \mathrm{cm}^{3}$, which is the lowest amongst this set of samples. The lowest skeletal density $\left(1.7226 \mathrm{~g} / \mathrm{cm}^{3}\right)$ was for a target density of $0.0970 \mathrm{~g} / \mathrm{cm}^{3}$. During the formation of the silica backbone by poly condensation in the gelation step, the lower target density provides more space where the crosslinking occurs in a more multidemensional fashion compared to the synthetic condition with higher target density. This spatial environment of the reaction system also offers less opportunity for the silianol groups to be interupted by byproducts from the gelation, such as excessive water and alchol (see Figure 18 in Chapter 3). As these two process conditions contribute to building the silica structure more than other parameters, they yield thicker growth of the braided silica network and higher skeletal density results.

Although the overall trends of the skeletal density was decreased with increasing target density, repetition of increasing and decreasing in skeletal densities were observed when they were compared side by side with change of the target density. The skeletal density were decreased over the range of target densities of $0.0194-0.0485 \mathrm{~g} / \mathrm{cm}^{3}$, but increased again for target densities between $0.0485-0.0776 \mathrm{~g} / \mathrm{cm}^{3}$. This pattern was repeated in futher observation of the next target density ranges, so the skeletal density was decreased in $0.0776-0.0970 \mathrm{~g} / \mathrm{cm}^{3}$ of the target density range and it was increased in $0.0970-0.1213 \mathrm{~g} / \mathrm{cm}^{3}$. Such repetition of trend change can be explained by considering the two processing parameters of the 
concentration of the gelation catalyst, that is ammonia in this research, and the amount of silanol that is associated with the target density. The gelation proceeds faster when the amount of silanol is increased and it influences the reaction rate more than the concentration of ammonia. However,

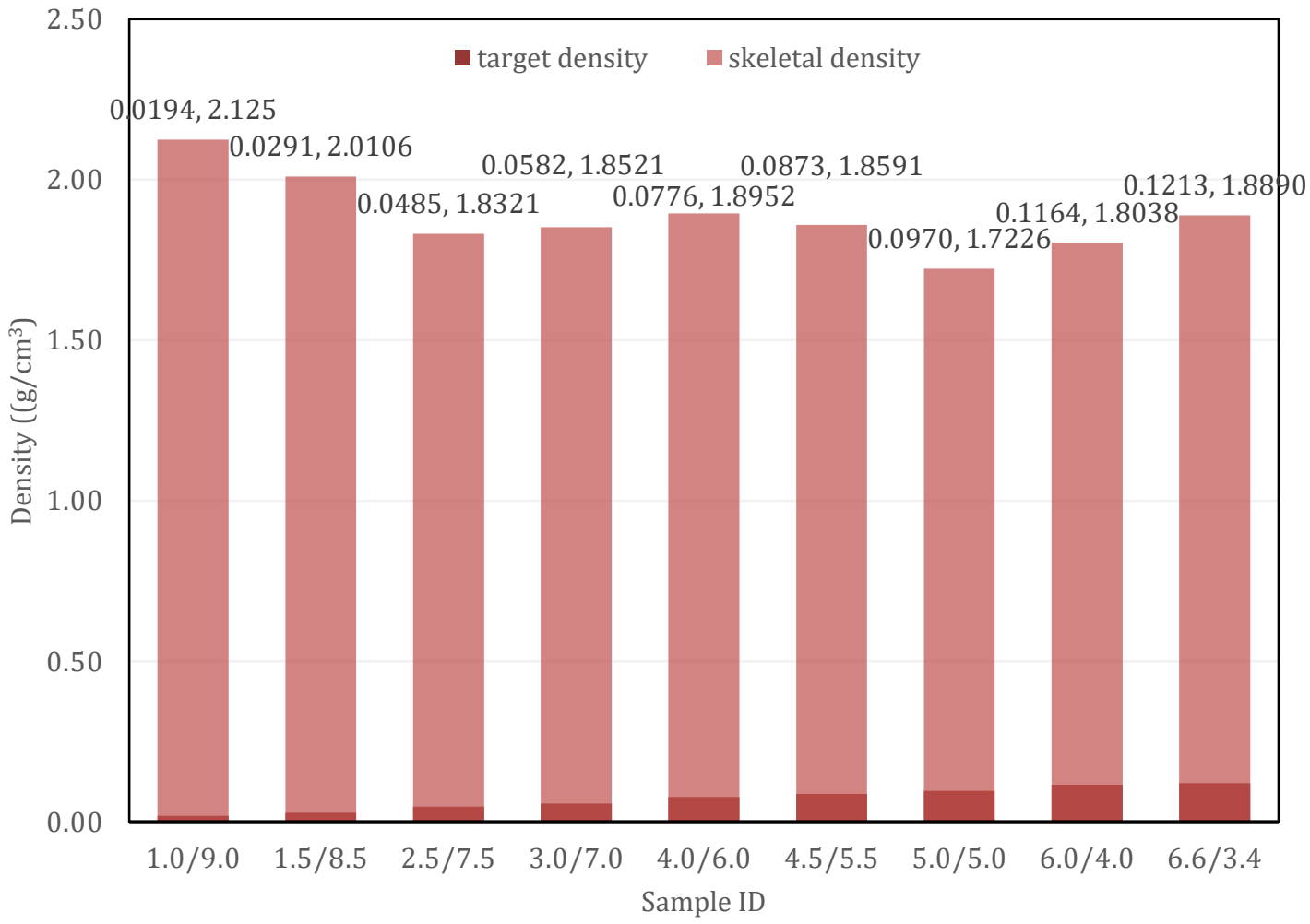

Figure 4-10. Effects of target density on skeletal density of monolithic silica aerogel (standard process). The left value of the data label is the target density $\left(\mathrm{g} / \mathrm{cm}^{3}\right)$ and the right one is the skeletal density $\left(\mathrm{g} / \mathrm{cm}^{3}\right)$ for each sample. The uncertainties are \pm $5 \%$ and $\pm 0.06 \%$ in target density and skeletal density respectively.

the gelation occurs rather slowly when the amount of silanol is too great for the applied amount of ammonia to catalyze the poly condensation promptly. Note again that this consideration can be applied when a relatively small range of target densiy of approximately $0.03 \mathrm{~g} / \mathrm{cm}^{3}$ is 
observed. At wider range, as investigated in this research, the trend in the changes in skeletal density decreased in an oscillating manner as the target density increased, as seen in Figure 4-10. Three duplicates of sample 3.0/7.0 and two of sample 4.0/6.0 were reproduced to confirm the results discussed, and the results where the same, within the given uncertainty $( \pm 5 \%$ and \pm $0.06 \%$ in target density and skeletal density respectively).

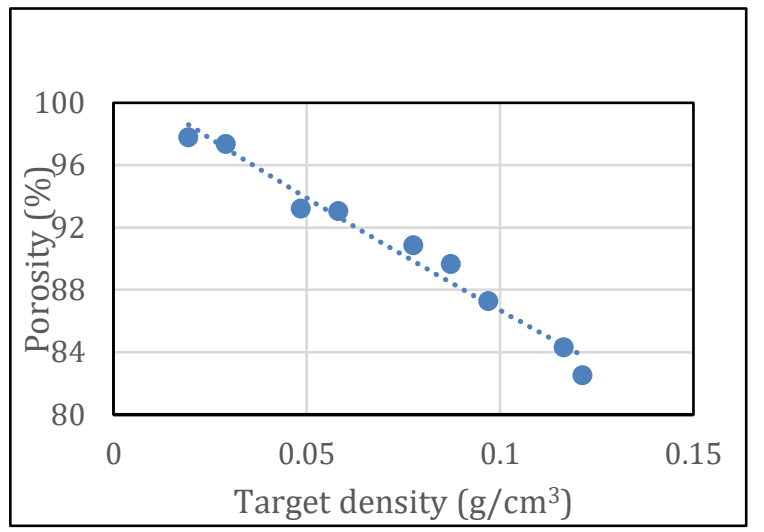

(a)

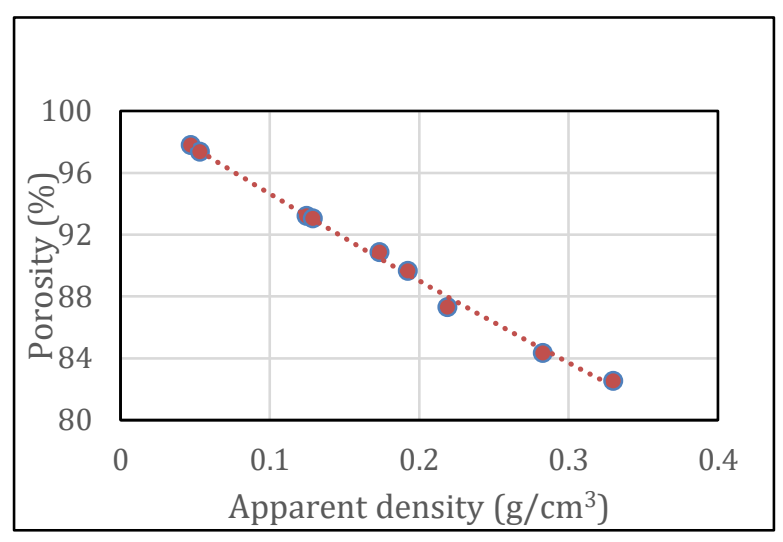

(b)

Figure 4-11. Porosity distributions for different target densities (left) and apparent densities of silica aerogels prepared from the standard process. The uncertainties are $\pm 5 \%, \pm 0.19 \%$, and $\pm 0.25 \%$ in target density, apparent density, and gelation time respectively. 


\subsubsection{Porosity changes with densities}

Samples ID\# 1.0/9.0, 1.5/8.5, 2.5/7.5, 3.0/7/0, 4.0/6.0, 4.5/5.5, 5.0/5.0, 6.0/4.0, and 6.6/3.4 were used to investigate the porosity changes with target, apparent and skeletal densities. The measured porosities of the silica aerogels produced using the standard process (the nitric acid - acetic acid combination for the DCM) are graphed with the target density and the apparent

Table 4-8. List of skeletal density and porosity of monolithic silica aerogels produced using the standard process. The uncertainties are $0.06 \%$ and $0.25 \%$ in skeletal density and porosity respectively.

\begin{tabular}{|c|c|c|}
\hline sample ID & $\begin{array}{c}\text { skeletal density } \\
\left(\mathrm{g} / \mathrm{cm}^{3}\right)\end{array}$ & $\begin{array}{c}\text { porosity } \\
(\%)\end{array}$ \\
\hline $1.0 / 9.0$ & 2.1250 & 97.798 \\
\hline $1.5 / 8.5$ & 2.0106 & 97.369 \\
\hline $2.5 / 7.5$ & 1.8321 & 93.211 \\
\hline $3.0 / 7.0$ & 1.8521 & 93.061 \\
\hline $4.0 / 6.0$ & 1.8952 & 90.860 \\
\hline $4.5 / 5.5$ & 1.8591 & 89.661 \\
\hline $5.0 / 5.0$ & 1.7226 & 87.296 \\
\hline $6.0 / 4.0$ & 1.8038 & 84.336 \\
\hline $6.6 / 3.4$ & 1.8890 & 82.543 \\
\hline
\end{tabular}


density, respectively, as shown in Figure 4-11. As expected theoretically, the target density is inversely proportional to the porosity throughout the range studied. The same holds for the apparent density. That is the lower target density or apparent density aerogels were the most porous. On the other hand, Table 4-8 shows the porosity as it varies with skeletal density, and the trend is less clear, but it appears the porosity increases with increasing skeletal density. This implies that the relationship between porosity and the two resulting densities (skeletal density and apparent density) was dominated by that of the porosity and the apparent density in this investigated range, although the porosity is calculated using both densities, as introduced in Chapter 2. The highest and the lowest porosities are $97.798 \%$ and $82.543 \%$ when the target densities are 0.01 and $0.12 \mathrm{~g} / \mathrm{cm}^{3}$, respectively.
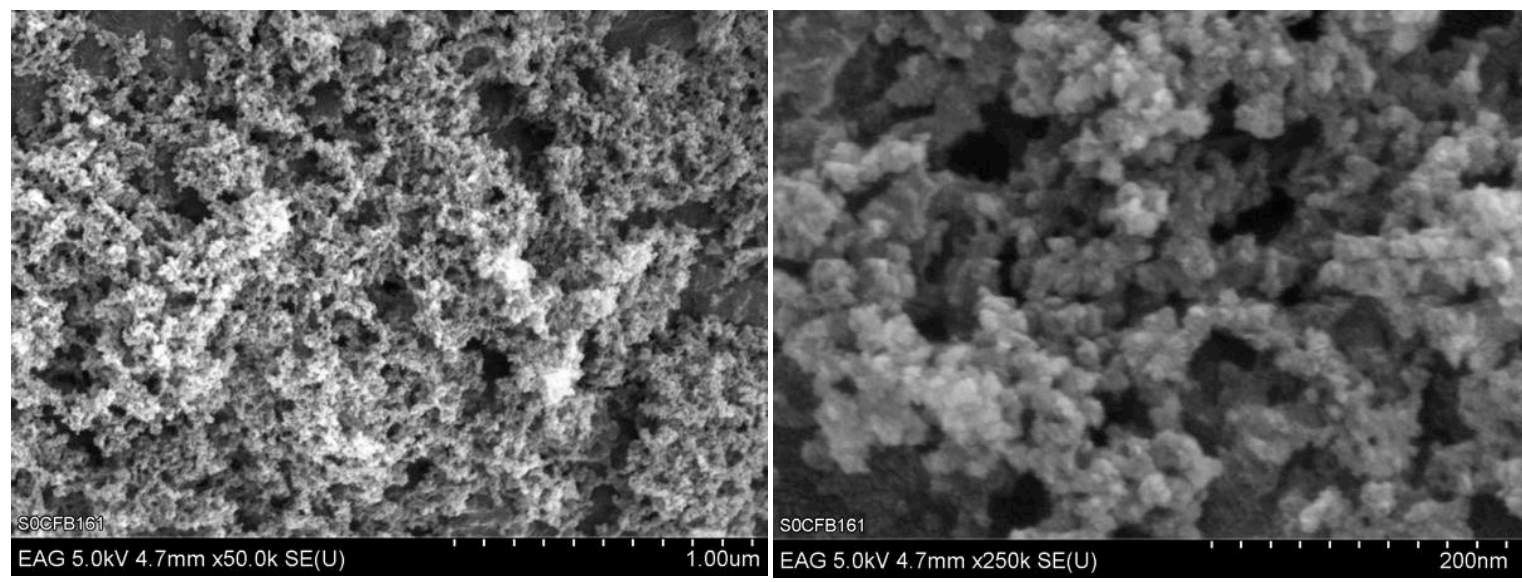

Figure 4-12. SEM photographs of 1.5/8.5, which has a low apparent density $\left(0.0529 \mathrm{~g} / \mathrm{cm}^{3}\right)$ and a high skeletal density $\left(2.0106 \mathrm{~g} / \mathrm{cm}^{3}\right)$. The monolithic silica aerogel was prepared by the standard process (magnification: left 1: 50,000, right 1: 250,000). 


\subsubsection{Discussion of morphological analysis from SEM pictures}

In addition to the measurements that have been discussed, scanning electron microscope
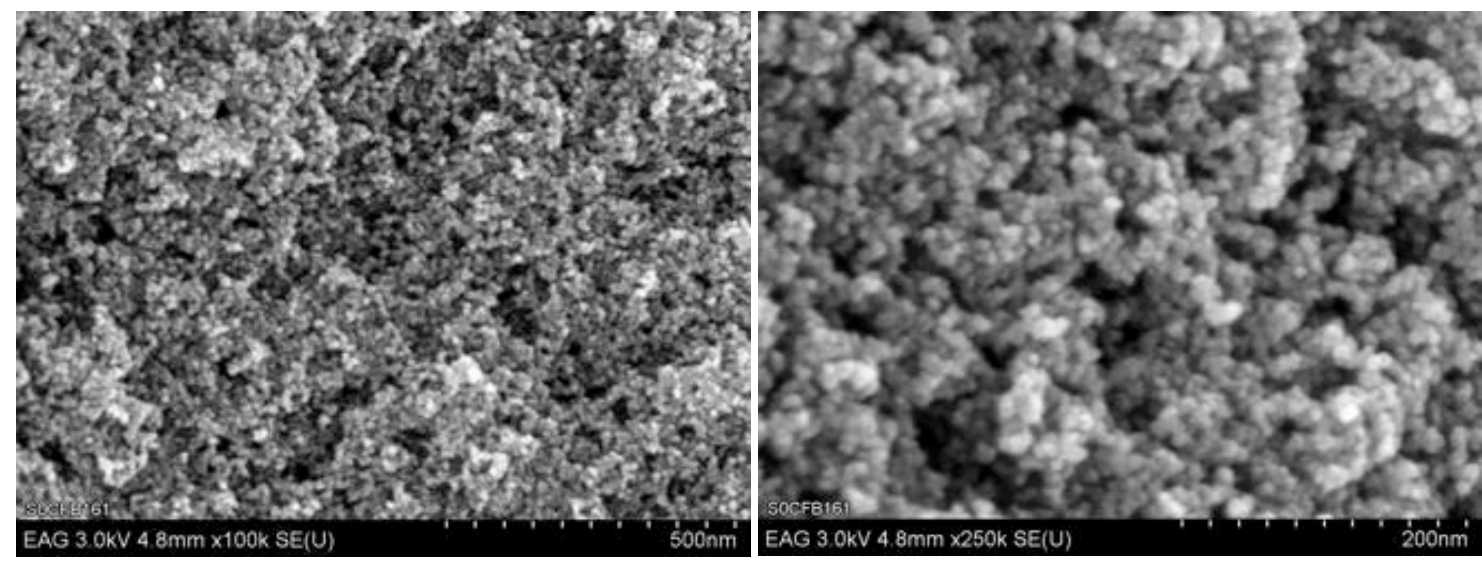

Figure 4-13. SEM photographs of 5.0/5.0, which has a high apparent density $\left(0.2188 \mathrm{~g} / \mathrm{cm}^{3}\right)$ and a low skeletal density $\left(1.7226 \mathrm{~g} / \mathrm{cm}^{3}\right)$. The monolithic silica aerogel was prepared by the standard process (magnification: left 1: 50,000, right 1: 250,000).

(SEM) images of some of the monolithic silica aerogel samples produced with the standard process in this research give more insight regarding the characterization results. Figure 4-12 illustrates SEM photographs of $1.5 / 8.5$, which has a low apparent density $0.0529 \mathrm{~g} / \mathrm{cm}^{3}$ and a high skeletal density of $2.2150 \mathrm{~g} / \mathrm{cm}^{3}$ (magnification: left 1: 50,000, right 1:250,000), whereas, Figure 4-13 shows those of 5.0/5.0, which has a high apparent density of $0.2188 \mathrm{~g} / \mathrm{cm}^{3}$ and a low skeletal density of $1.7226 \mathrm{~g} / \mathrm{cm}^{3}$. It can be observed that the overall space (pores) of the aerogel network is larger in the sample with the low apparent density as compared to that with the higher apparent density case (see the photos of low magnifications), even considering the differing magnifications, which were necessary to obtain the best quality images. This provides evidence that the corresponding aerogel has a relatively higher porosity. 
On the other hand, the photos with high magnification $(1: 250,000)$ on both aerogels clearly show how the silica backbones were formed as discussed previously. The crosslinks are made from highly irregular shapes of silica spheres that create relatively fewer voids between themselves, and it causes the corresponding aerogel to exhibit a higher skeletal density. We can see how homogeneously the silica spheres are formed and cross-linked with each other in Figure 4-13. This uniqueness in the textural property can again be taken as evidence that the corresponding silica aerogel monolith has higher transparency, as discussed earlier, as it provides higher chance for the pores to be more uniformly sized.

\subsubsection{Effects of processing conditions on porosity}

Changes were made to some of the process parameters for another series of silica aerogels which were synthesized in order to investigate the influence of these processing conditions on the resulting textural properties, mainly focusing on the porosity. Different catalysts for the DCM step, and several combinations of gelation diluents were employed and the produced silica aerogels were compared side by side with the sample prepared by the standard process (3.0/7.0).

\subsubsection{Effects of solvent types on porosity}

Methanol and ethanol-water mixtures replaced the ethanol in the gelation step as a diluent while all other parameters remained identical to those in the standard process at a target density of $0.0582 \mathrm{~g} / \mathrm{cm}^{3}$. As illustrated in Table 4-9, all solvents produced higher apparent densities and higher skeletal densities, as well as increased gelation times compared to the standard sample. 
The porosity, on the other hand, was decreased by approximately $2 \%$ (from $98.061 \%$ to $91.267 \%$ or $91.714 \%$ ) when methanol was employed while those with the ethanol-water mixtures showed no significant difference from that of the standard sample. These results show that when methanol is used, the change in the apparent density governs the porosity because the change in the skeletal density is relatively small. When water is added to the conventional diluent (ethanol), on the other hand, the changes in both densities affect the porosity at a similar level and thus the resulting porosities stayed nearly the same as that of the standard one. This can be explained by the fact that esterification controls the formation of the aerogel pores predominantly. This reverse reaction of the poly condensation occurs during and after the gelation due to the presence of solvent with different mother liquor from the precursor (TEOS). This results in the demonstrated differences in the measured densities as well as the longer gelation times compared to that of the standard process. The presence of water in the diluent affects the gelation reaction and the formation of the pores as well because it hinders the overall chemical reactions during the gelation step. The influence, however, is not sufficiently great to change the nature of porosity as observed in Table 4-9. 
Table 4-9. Effects of solvent types in gelation on densities and porosities of monolithic silica aerogels.

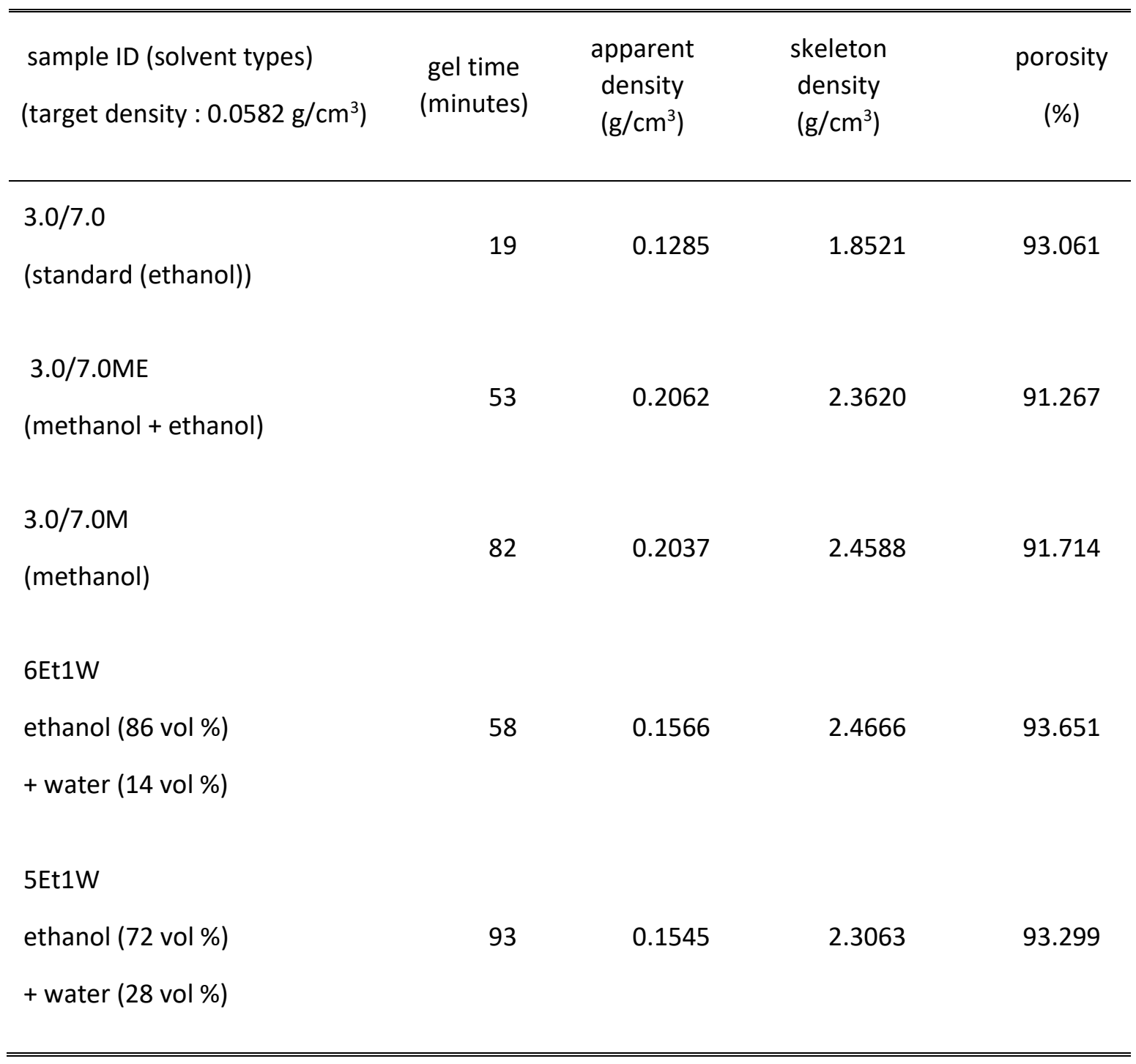


Figure 4-14 shows how the pore size distributions differed between methanol-gelation, methanol-ethanol mixture gelation, and ethanol-gelation. The pore size distribution got narrower and was shifted to smaller sizes when methanol was employed. This difference is also well reflected in the average pore diameters as illustrated in Table 4-10. Figure 4-15 displays the other comparison of the pore size distribution between ethanol-water mixtures and ethanol gelation. While all of the samples using ethanol-water mixtures show quite similar pore size distribution widths, it can be observed that the pore size distribution is slightly decreased with a larger volume $\%$ of water in the diluent. Table $4-10$ also shows the surface areas and the pore

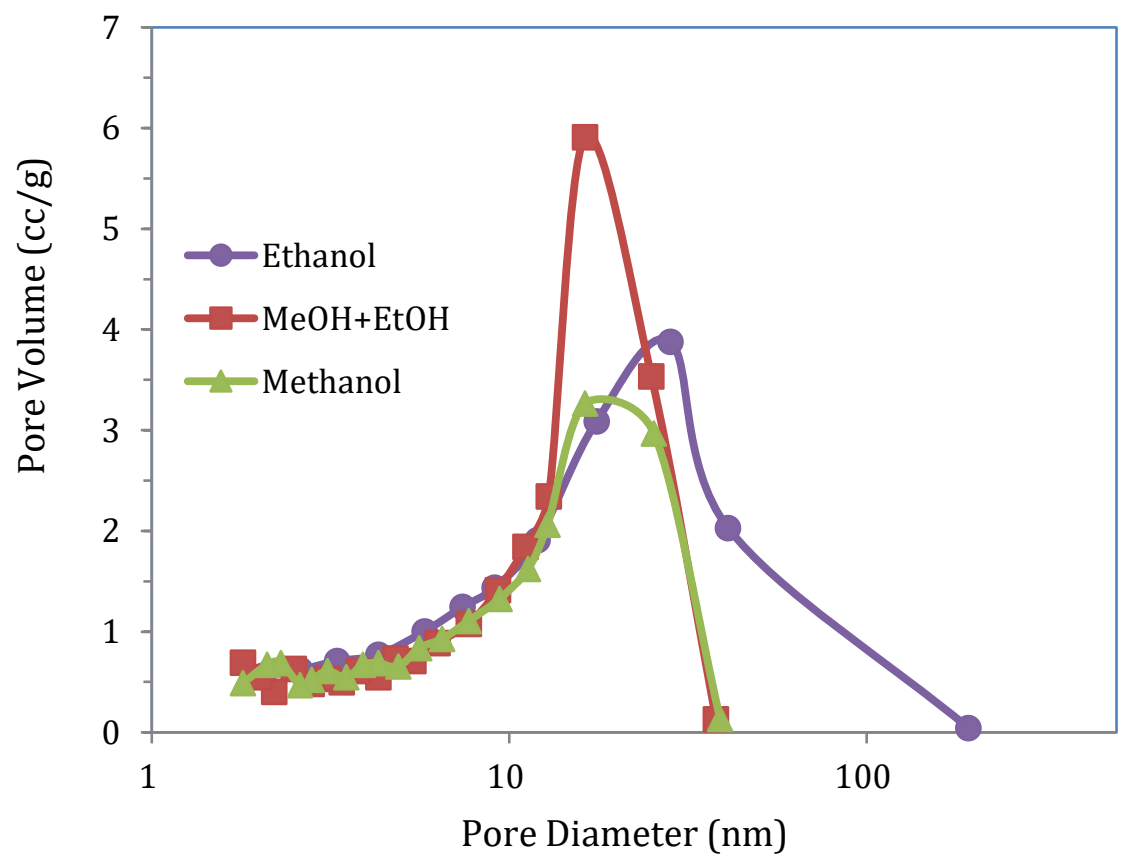

Figure 4-14. Effects of solvent types in gelation step on pore size distributions of monolithic silica aerogels. The pre-hydrolyzed sol was prepared by the standard DCM (nitric acid - acetic acid combination). 
volumes of the discussed samples with unremarkable differences between them (surface area : $800 \sim 950 \mathrm{~m}^{2} / \mathrm{g}$, pore volume : $2.02 \sim 2.63 \mathrm{cc} / \mathrm{g}$ ).

Table 4-10. Effects of solvent types on textural properties of monolithic silica aerogels

\begin{tabular}{|c|c|c|c|}
\hline sample ID (solvent types) & $\begin{array}{r}\text { BET } \\
\text { surface area } \\
\left(\mathrm{m}^{2} / \mathrm{g}\right)\end{array}$ & $\begin{array}{c}\text { pore volume } \\
\left(\mathrm{N}_{2} \text { desorption) }\right. \\
\text { (cc/g) }\end{array}$ & $\begin{array}{l}\text { avg. pore } \\
\text { diameter } \\
(\mathrm{nm})\end{array}$ \\
\hline $\begin{array}{l}\text { 3.0/7.0) } \\
\text { (standard (ethanol)) }\end{array}$ & 955.2 & 2.63 & 10.33 \\
\hline $\begin{array}{l}\text { 3.0/7.0ME } \\
\text { (methanol + ethanol) }\end{array}$ & 903.5 & 2.60 & 10.22 \\
\hline $\begin{array}{l}\text { 3.0/7.0M } \\
\text { (methanol) }\end{array}$ & 807.3 & 2.02 & 9.06 \\
\hline $\begin{array}{l}6 \text { Et1W } \\
\text { ethanol ( } 86 \text { vol \%) } \\
+ \text { water (14 vol \%) }\end{array}$ & 950.0 & 2.61 & 9.72 \\
\hline $\begin{array}{l}\text { 5Et1W } \\
\text { ethanol (72 vol \%) }\end{array}$ & 884.0 & 2.42 & 9.91 \\
\hline + water (28 vol \%) & & & \\
\hline
\end{tabular}




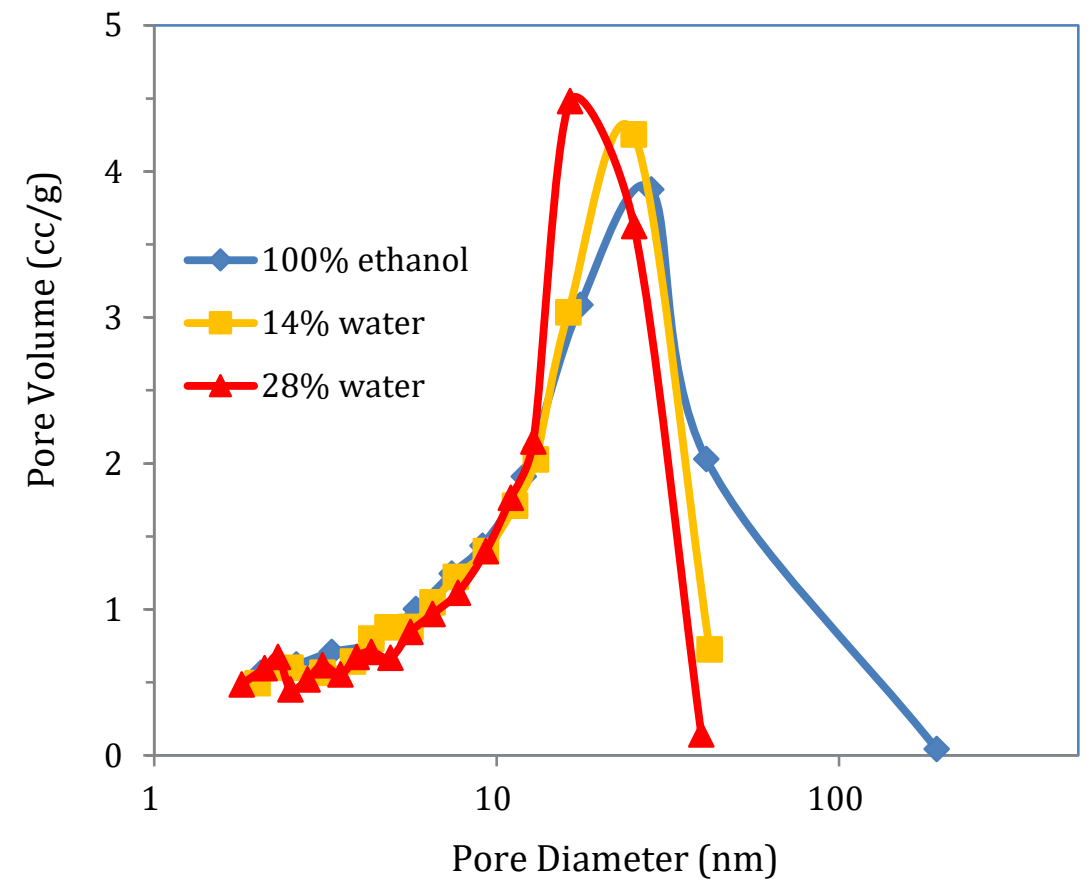

Figure 4-15. Effects of adding water in the gelation step on the pore size distributions of monolithic silica aerogels. The pre-hydrolyzed sol was prepared by the standard DCM (nitric acid - acetic acid combination).

\subsubsection{Effects of catalyst type on porosity}

The sulfuric acid - acetic acid combination was preliminarily investigated as part of the development journey of the DCM and it was demonstrated that use of this combination works to produce pre-hydrolyzed sol from homogeneous hydrolysis. As illustrated in Table 4-11, the resulting aerogel has higher apparent density and skeletal density and consequently lower porosity by $2 \%(91.862 \%$ versus $93.061 \%)$, along with a considerably longer gelation time when compared to the standard sample. This difference is primarily related to the $\mathrm{pH}$ difference of the produced sol as reported in Chapter 3. The more acidic sol prepared by the sulfuric acid - acetic 
Table 4-11. Effects of pre-hydrolysis catalyst on densities and porosities of monolithic silica aerogels.

\begin{tabular}{lcccc}
\hline \hline $\begin{array}{l}\text { pre-hydrolysis catalyst } \\
\text { (target density : 0.0582 } \mathrm{g} / \mathrm{cm}^{3} \text { ) }\end{array}$ & $\begin{array}{c}\text { gel time } \\
\text { (minutes) }\end{array}$ & $\begin{array}{c}\text { apparent } \\
\text { density }\left(\mathrm{g} / \mathrm{cm}^{3}\right)\end{array}$ & $\begin{array}{c}\text { skeleton } \\
\text { density }\left(\mathrm{g} / \mathrm{cm}^{3}\right)\end{array}$ & porosity (\%) \\
\hline sample ID:3.0/7.0 & 19 & 0.128 & 1.8521 & 93.061 \\
(nitric acid-acetic acid) & & & & \\
sample ID:SA3.0/7.0 & 52 & 0.175 & 2.1533 & 91.862 \\
sulfuric acid-acetic acid & & & & \\
\hline \hline
\end{tabular}

acid combination contributes to forming smaller pores in the gel network, which implies thicker growth of the silica networks. This also causes longer gelation times as the gelation takes place under a base catalyzing environment with ammonia as a catalyst.

Figure 4-16 shows the pore size distribution and it also agrees with what is observed from Table 4-11 as the main pore area is shifted to a smaller size from that of the standard sample. Such characteristic differences, however, are not clearly observed in the textural properties characterized by the $\mathrm{N}_{2}$ adsorption-desorption method as displayed in Table 4-12. This is a good example of how accurately the helium pycnometer enables characterization of the extremely nano-porous aerogel structure. 


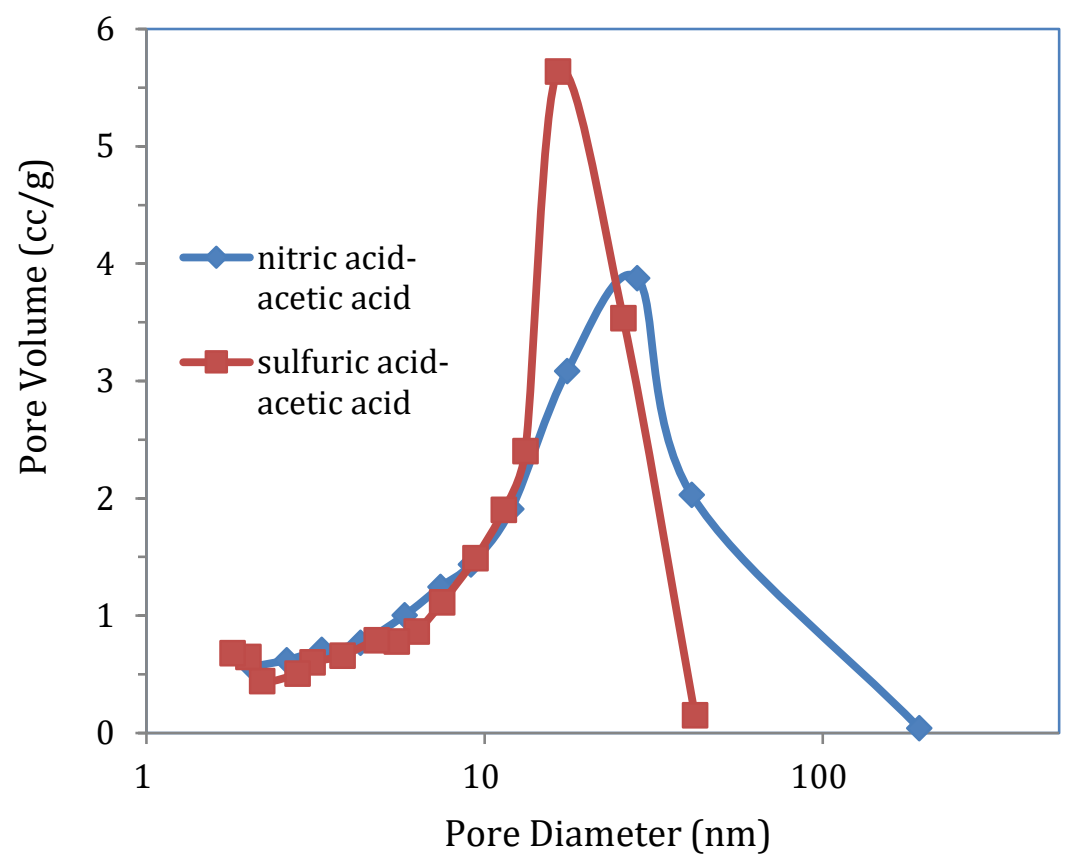

Figure 4-16. Change in pore size distribution of silica aerogel synthesized by sulfuric acid - acetic acid combination using the DCM.

Table 4-12. Effects of pre-hydrolysis catalyst on textural properties of monolithic silica aerogels.

\begin{tabular}{lccc}
\hline $\begin{array}{l}\text { pre-hydrolysis catalyst } \\
\left.\text { (target density : } 0.0582 \mathrm{~g} / \mathrm{cm}^{3}\right)\end{array}$ & $\begin{array}{c}\text { BET surface area } \\
\left(\mathrm{m}^{2} / \mathrm{g}\right)\end{array}$ & $\begin{array}{c}\text { pore volume } \\
\left(\mathrm{N}_{2} \text { desorption) }\right. \\
(\mathrm{cc} / \mathrm{g})\end{array}$ & $\begin{array}{c}\text { avg. pore } \\
\text { diameter } \\
(\mathrm{nm})\end{array}$ \\
\hline sample ID:3.0/7.0 & 955.2 & 2.63 & 10.33 \\
(nitric acid-acetic acid) & & & 10.29 \\
sample ID:SA3.0/7.0 & 934.7 & 2.73 & \\
sulfuric acid-acetic acid & & & \\
\hline \hline
\end{tabular}




\subsubsection{Effects of hydrophobicity on porosity}

Hydrophobic silica aerogel was prepared by aging the wet gel in an aging solution prepared using $\operatorname{HMDZ}\left(1,1,3,3\right.$-hexamethyldisilazine) diluted in ethanol at $328 \mathrm{~K}\left(55^{\circ} \mathrm{C}\right)$ for 6 hours. Figure 4-17 illustrates photos of the hydrophobic aerogel monolith with water droplets on it
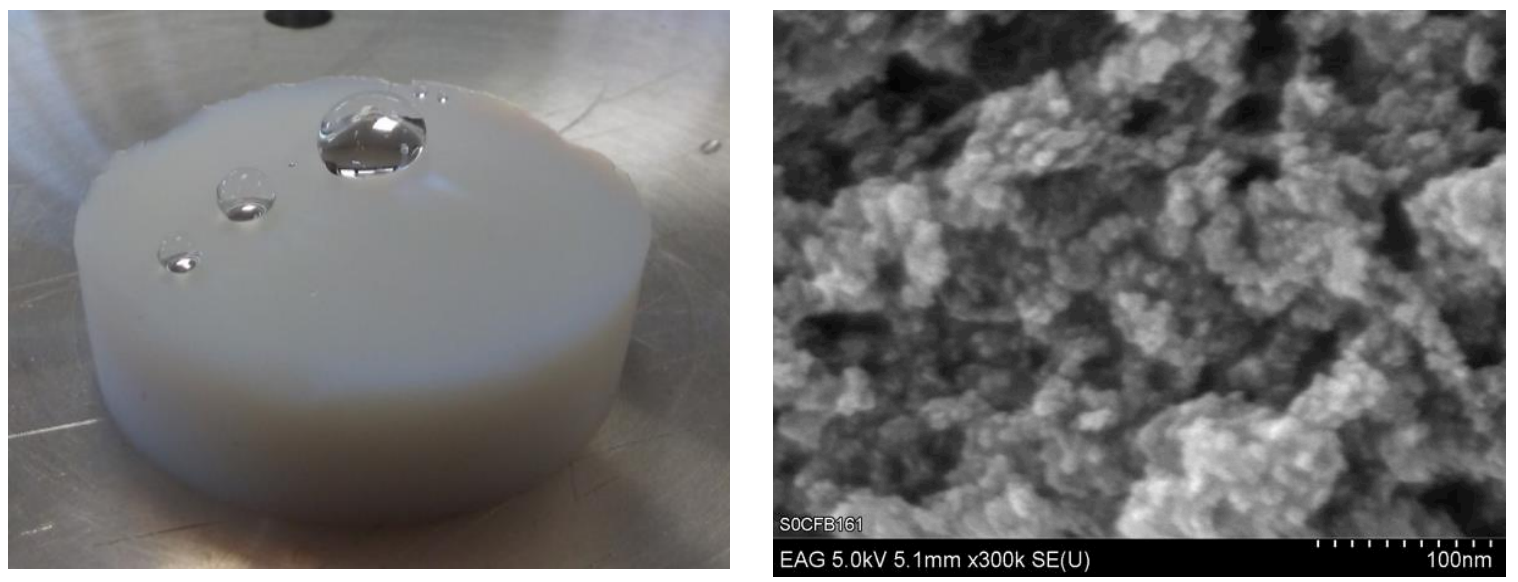

Figure 4-17. Water droplet on the hydrophobic silica aerogel (left) and SEM photographs of hydrophobic silica aerogel (right 1:300,000).

(left) and the textural feature of hydrophobic silica aerogel (SEM photo, right). Unlike that of the hydrophilic aerogel shown in Figure 4-12, the spaces between the spherical silica particles are filled, resulting in more clustered groups forming in the structure. This is due to the presence of the methyl group that is known to create strong hydrophobicity by replacing the hydroxyl groups of the silanol $(\mathrm{SiO}(\mathrm{OH}))$ groups, a remnant of a typical sol-process, which are unstable at the ends of the silica chains.

Table 4-13 lists gelation times, densities, and porosities of the hydrophobic sample compared to the hydrophilic one (the standard sample). The gelation times recorded were nearly identical because the synthetic route was the same (19 minutes and 18 minutes). While the 
apparent densities measured showed a negligible difference $\left(0.128\right.$ vs $\left.0.133 \mathrm{~g} / \mathrm{cm}^{3}\right)$, clear differences were observed from the skeletal densities and this resulted in a $2 \%$ lower porosity

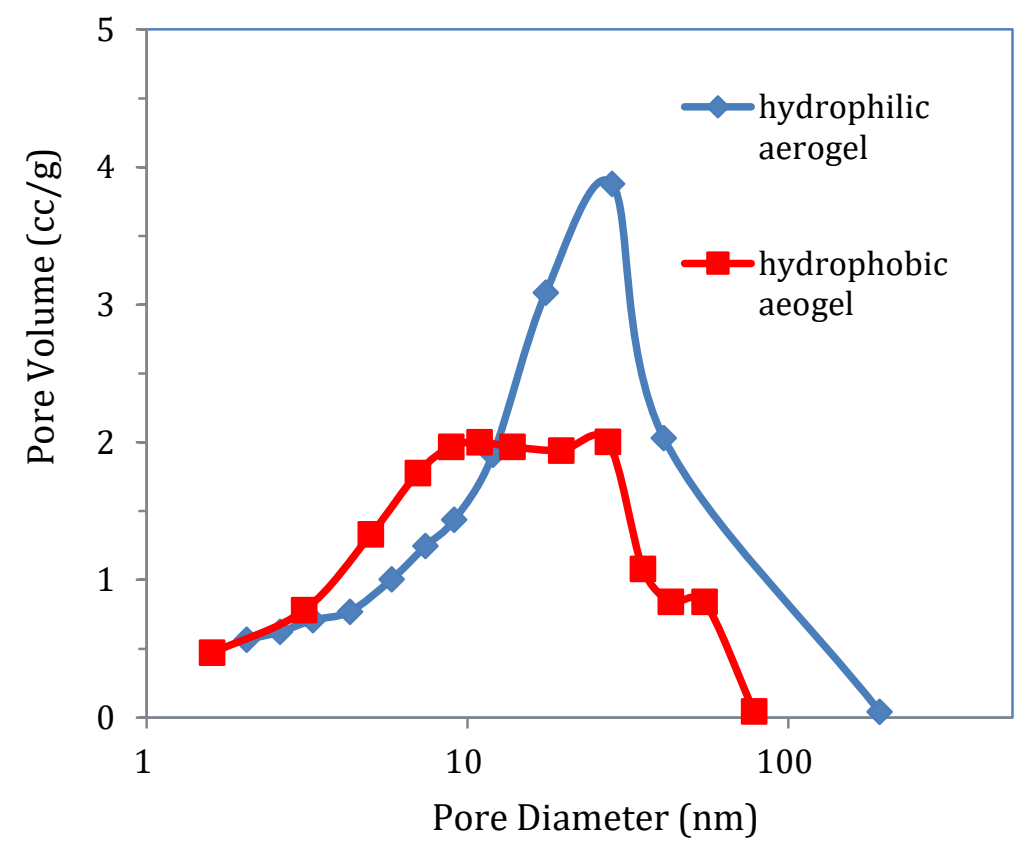

Figure 4-18. Change in pore size distribution of silica aerogel with hydrophobicity.

for the hydrophobic aerogel monolith (91.266\% versus 93.061\%). Pore size distribution changes can be observed from Figure 4-18. The main distribution of the pore sizes becomes broader with a shift toward smaller diameters compared to that of the standard sample. Similar differences are also found in the average pore diameters listed in Table 4-14 together with the surface areas and the pore volumes, all of which show decreases compared to the hydrophilic one (the standard sample). 
Table 4-13. Effects of aging treatment for hydrophobicity on densities and porosities of monolithic silica aerogels.

\begin{tabular}{lcccc}
\hline \hline $\begin{array}{l}\text { sample property } \\
\left.\text { (target density : } \mathbf{0 . 0 5 8 2} \mathbf{g} / \mathrm{cm}^{3}\right)\end{array}$ & $\begin{array}{c}\text { gel time } \\
\text { (minutes) }\end{array}$ & $\begin{array}{c}\text { apparent } \\
\text { density }\left(\mathrm{g} / \mathrm{cm}^{3}\right)\end{array}$ & $\begin{array}{c}\text { skeleton } \\
\text { density }\left(\mathbf{g} / \mathrm{cm}^{3}\right)\end{array}$ & $\begin{array}{c}\text { porosity } \\
\text { (\%) }\end{array}$ \\
\hline sample ID:3.0/7.0 & 19 & 0.128 & 1.8521 & 93.061 \\
(hydrophilic aerogel) & & & & \\
sample ID:HB3.0/7.0 & 18 & 0.133 & 1.5311 & 91.266 \\
(hydrophobic aerogel) & & & & \\
\hline \hline
\end{tabular}

Table 4-14. Effects of hydrophobicity on textural properties of monolithic silica aerogels.

\begin{tabular}{lccc}
\hline $\begin{array}{l}\text { sample property } \\
\left.\text { (target density }: \mathbf{0 . 0 5 8 2} \mathbf{g} / \mathrm{cm}^{3}\right)\end{array}$ & $\begin{array}{c}\text { BET surface area } \\
\left(\mathrm{m}^{2} / \mathrm{g}\right)\end{array}$ & $\begin{array}{c}\text { pore volume } \\
\left(\mathbf{N}_{\mathbf{2}} \text { desorption) }\right. \\
(\mathbf{c c} / \mathrm{g})\end{array}$ & $\begin{array}{c}\text { avg. pore } \\
\text { diameter } \\
(\mathbf{n m})\end{array}$ \\
\hline sample ID:3.0/7.0 & 955.2 & 2.63 & 10.33 \\
(hydrophilic aerogel) & & & \\
sample ID:HB3.0/7.0 & 877.2 & 2.17 & 8.16 \\
(hydrophobic aerogel) & & & \\
\hline \hline
\end{tabular}




\subsection{Conclusions}

A series of monolithic silica aerogels were produced using the two step sol-gel process, an aging step, and the $\mathrm{CO}_{2}$ supercritical drying process (the COLD method). The newly developed pre-hydrolysis method (direct contact method, DCM) was successfully applied to the two step sol-gel route and demonstrated great feasibility for enabling tuning of the synthetic conditions to achieve a desired target property of the silica aerogel texture, specifically in terms of the porosity. The solvent type at the gelation step, the catalyst type for the DCM process, and the aging treatment used to establish hydrophobicity in the aerogel all affect the porosity of the produced aerogel, as well as other textural properties. From this research, it has been demonstrated that a silica aerogel with a desired porosity can be obtained by appropriately adjusting the processing parameters. With this control of porosity, the ability to optimize the thermal conductivity of the produced silica aerogel for specific applications with minimal trials is enabled. This makes employing the DCM in the aerogel production process highly desirable. 


\section{Chapter 5}

\section{Synthesis and Porosity Study of Yttria Aerogels}

\section{and Organic Aerogels}

This chapter describes the study of yttria aerogel and organic aerogel. For the yttria aerogel, the synthetic route that produces monolithic samples was extensively investigated and the processing condition yielding successful product is introduced. Monolithic yttria aerogels were produced by this selected set of processing conditions at 5 different target densities and characterized by BJH, BET and the porosity measurement. The organic aerogels were prepared using a combination of phloroglucinol - formaldehyde (PF) as the precursors. Like the yttria aerogels, 5 target densities were set as the synthetic conditions and the same characterizations for the yttria aerogels were performed on these organic aerogels and summarized. The porosity studies of these aerogels provide the basic information in terms of the relationship between the synthetic condition and the resulting porosity as no public report has been released on this topic.

\subsection{Monolithic Yttria Aerogel}

It had been a challenge to synthesize metal oxide aerogel monoliths from metal alkoxide precursors because the conditions required for the sol-gel process were limited to those leading to formation of a gel texture that was polymeric and mechanically stable. However, in 2004 Gash et al. reported that use of organic epoxide with an alcoholic solution of metal ion salts 
enables synthesis of homogeneously structured metal oxide alcogel [7]. In this sol-gel process, the organic epoxide acts as a proton scavenger that induces the metal ions to undergo hydrolysis and uniform condensation to form a metal oxide sol with substituted alcohol species forming as the side product of ring-opening.

Although it was proven that the sol-gel process using metal salt precursors and epoxide was promising for production of metal oxide monolithic aerogels, further investigations still remain to determine the optimum sol-gel route for each monolithic metal oxide aerogel. In this dissertation research, a preliminary investigation was performed to develop a sol-gel process for synthesis of yttria aerogel $\left(\mathrm{Y}_{2} \mathrm{O}_{3}\right)$. Yttria has high thermal stability at high temperature $\left(\sim 1000^{\circ} \mathrm{C}\right)$ with minimum change in its inherent structure so it is considered to be a candidate as a key material in the high temperature thermal protection system used for the NASA space shuttle [117]. In addition, $\mathrm{Y}_{2} \mathrm{O}_{3}$ doped with rare earth metals yields interesting luminescent oxide materials. Notably Eu doped $\mathrm{Y}_{2} \mathrm{O}_{3}$ is one of the best luminophores regarding the emission efficiency of red light. Many publications have addressed the properties of these materials, often made by traditional ceramics processing $[118,119,120]$.

\subsubsection{Experimental method}

Yttrium chloride hexahydrate $\left(\mathrm{YCl}_{3} \cdot 6 \mathrm{H}_{2} \mathrm{O}\right)$ was selected as a precursor because it has good solubility in alcoholic solvent and water. Propylene oxide plays the role of the proton scavenger and both methanol and ethanol were tested as solvents. Table 5-1 introduces the reagents employed in this synthesis investigation. 
$\mathrm{YCl}_{3} \cdot 6 \mathrm{H}_{2} \mathrm{O}$ was dissolved in a mixture of ethanol, methanol, and water whose molar ratios were optimally adjusted according to the predetermined target densities whose range was 0.053 $-0.069 \mathrm{~g} / \mathrm{cm}^{3}$ with intervals of $0.003-0.005 \mathrm{~g} / \mathrm{cm}^{3}$. After complete dissolution, a pre-quantified amount of propylene oxide was slowly added to the $\mathrm{YCl}_{3} \cdot 6 \mathrm{H}_{2} \mathrm{O}$ solution to control the reaction rate. This quantification of propylene oxide was determined so that the gelation time was approximately 50 minutes. This length of the gelation time was proven to be appropriate for the sol-to-gel reaction speed to build a homogeneous crosslinking of the yttria chains from the

Table 5-1. List of reagents used for yttria aerogel production.

\begin{tabular}{lccccc}
\hline \hline Reagent & Formula & Mol. wt. & $\begin{array}{c}\text { Density } \\
\left(\mathbf{g} / \mathbf{c m}^{3}\right)\end{array}$ & $\begin{array}{c}\text { Boiling Point } \\
\left({ }^{\circ} \mathrm{C}\right)\end{array}$ & Supplier \\
\hline $\begin{array}{l}\text { Yttrium(III) } \\
\begin{array}{l}\text { Chloride } \\
\text { hexahydrate }\end{array}\end{array}$ & $\mathrm{YCl}_{3} \cdot 6 \mathrm{H}_{2} \mathrm{O}$ & 303.36 & 2.18 & $\begin{array}{c}100 \\
\text { (melting point) }\end{array}$ & Aldrich \\
$\begin{array}{l}\text { Ethanol } \\
\mathrm{C}_{2} \mathrm{H}_{5} \mathrm{OH}\end{array}$ & 46.07 & 0.79 & 78.1 & McMaster \\
$\begin{array}{l}\text { Methanol } \\
\mathrm{CH}_{3} \mathrm{OH}\end{array}$ & 32.04 & 0.79 & 64.7 & Aldrich \\
$\begin{array}{l}\text { Propylene } \\
\text { oxide }\end{array}$ & $\mathrm{C}_{3} \mathrm{H}_{6} \mathrm{O}$ & 58.08 & 0.829 & $33-34$ & Aldrich \\
\hline \hline
\end{tabular}

preliminary experiment. This sol was vigorously stirred and transferred to a disk type of mold, which had an inner diameter of $30 \mathrm{~mm}$. The sol in the mold was gelled for a certain amount of time, which was depending upon the specified target density and continued strengthening its structure for the next 45 to 50 minutes. Over the planned range of the target density, the sol-gel condition for the lower target density required relatively longer gelation time than that for the 
higher target density. The results of the gelation time are presented in the next section of results and discussion.

The synthesized wet gels were transformed into aerogels by the COLD method for the supercritical drying process as described in Chapter 4 for the silica aerogel production. The produced yttria aerogels textural properties were characterized by measuring the BET surface areas and the $\mathrm{BJH}$ pore volumes and pore diameters. The skeletal densities were measured using the He pycnometer to estimate the porosities of the samples.

(a) $3 \mathrm{Cl}-+\mathrm{CH}_{3} \mathrm{CH}-\mathrm{CH}_{2}+\left[\mathrm{Y}\left(\mathrm{H}_{2} \mathrm{O}\right)_{6}\right]^{3+}$

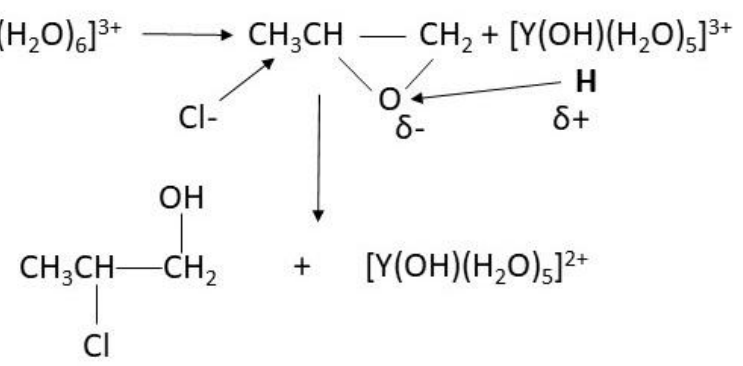

(b) $2\left[\mathrm{Y}(\mathrm{OH})\left(\mathrm{H}_{2} \mathrm{O}\right)_{5}\right]^{2+} \longleftrightarrow\left[\left(\mathrm{H}_{2} \mathrm{O}\right)_{5} \mathrm{YOY}\left(\mathrm{H}_{2} \mathrm{O}\right)_{5}\right]^{4+}+\mathrm{H}_{2} \mathrm{O}$

$$
\left[\left(\mathrm{H}_{2} \mathrm{O}\right)_{5} \mathrm{YOY}\left(\mathrm{H}_{2} \mathrm{O}\right)_{5}\right]^{4+}+2 \mathrm{H}_{2} \mathrm{O} \longrightarrow \mathrm{Y}_{2} \mathrm{O}_{3}+10 \mathrm{H}_{2} \mathrm{O}+\mathrm{H}^{4+}
$$

Figure 5-1. The deprotonation and poly-condensation of reaction mechanism for $\mathrm{Y}_{2} \mathrm{O}_{3}$ alcogel synthesis. Chemical equations are based on Reference [121]. 


\subsubsection{Results and discussion}

Figure 5-1 briefly describes the synthetic production route for yttiria alcogel when $\mathrm{YCl}_{3} \cdot 6 \mathrm{H}_{2} \mathrm{O}$ and propylene oxide are used as the precursor and epoxide respectively. With chlorine anions, a strong Lewis base, a nucleophilic attack on the secondary carbon of the epoxide, increases the negative partial charge on the epoxide oxygen according to the reaction mechanism (a). The latter can in turn attack and capture one proton in a coordination water molecule of the cation,

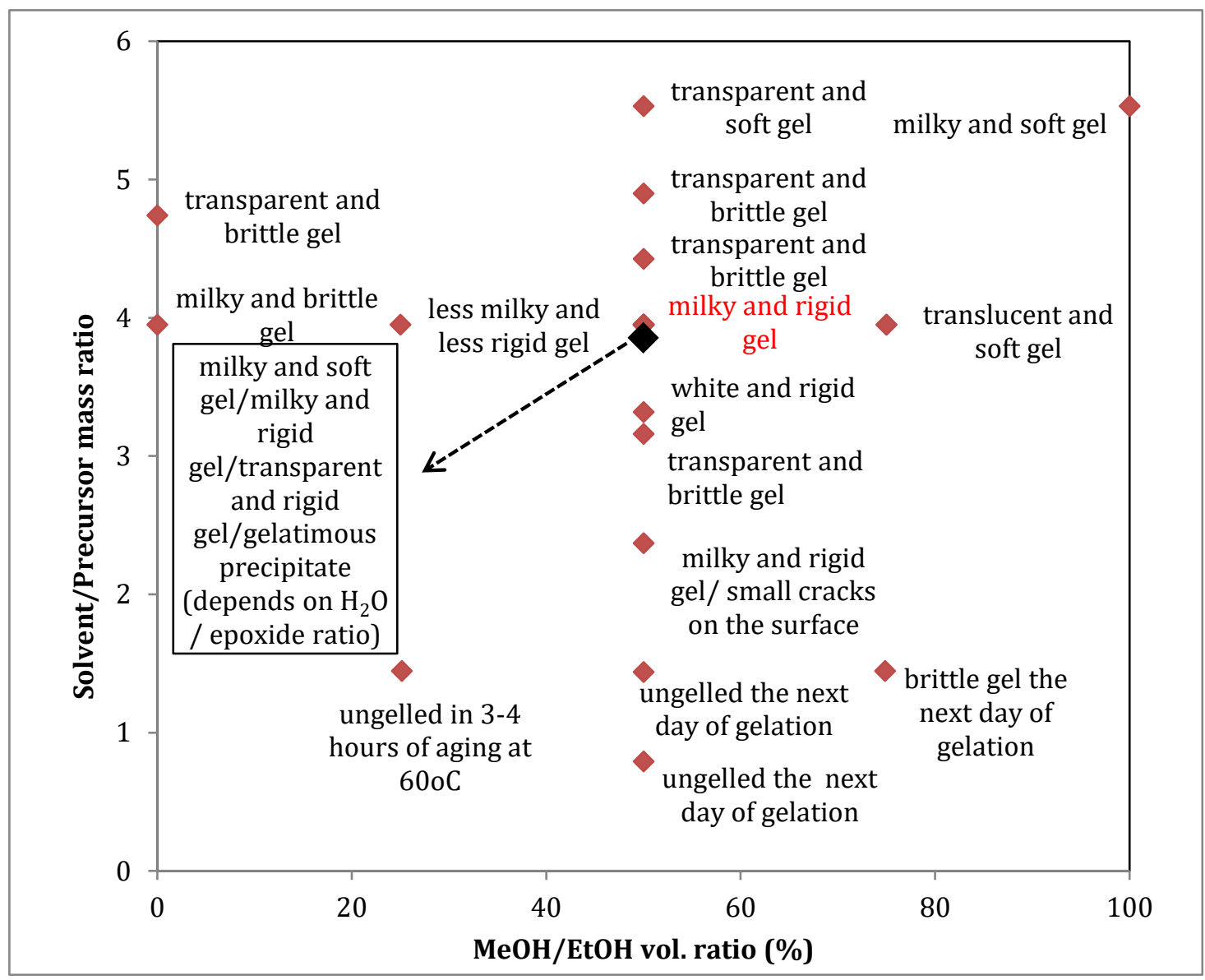

Figure 5-2. Summary of the sol-gel investigation and resultant gel states of yttria alcogel prepared by yttrium hexahychloride and propylene oxide in alcoholic solvent environment. 
which leaves a hydrolyzed cation carrying a hydroxo ligand. This reaction can be expected to be largely dependent upon the nature of the anion in the metallic salt. Condensation reactions of type (b) can then proceed and end in the formation of a $\mathrm{Y}_{2} \mathrm{O}_{3}$ gel [121].

Effects of solvent types, the amount of water, the amount of the epoxide, and volumetric ratios between the reactants have been actively investigated and a robust sol-gel process has been set up to produce monolithic yttria alcogel. Figure 5-2 shows a summary of the investigation to finalize the sol-gel route to form a yttria alcogel monolith. During the

Table 5-2. Resulting gelation time and corresponding densities of the monolithic yttria aerogels.

\begin{tabular}{lcccc}
\hline \hline Sample ID & $\begin{array}{c}\text { gelation time } \\
(\mathrm{min})\end{array}$ & $\begin{array}{c}\text { target density } \\
\left(\mathrm{g} / \mathrm{cm}^{3}\right)\end{array}$ & $\begin{array}{c}\text { apparent } \\
\text { density }\left(\mathrm{g} / \mathrm{cm}^{3}\right)\end{array}$ & $\begin{array}{c}\text { skeleton } \\
\text { density }\left(\mathrm{g} / \mathrm{cm}^{3}\right)\end{array}$ \\
\hline $10-10$ & 41 & 0.069 & 0.095 & 2.548 \\
$11-11$ & 43 & 0.065 & 0.092 & 2.524 \\
$12-12$ & 52 & 0.060 & 0.088 & 2.630 \\
$13-13$ & 58 & 0.057 & 0.085 & 2.719 \\
$14-14$ & 65 & 0.053 & 0.082 & 2.667 \\
\hline \hline
\end{tabular}

investigation for the solvent effects, it was found that use of methanol yields transparent and brittle gels whereas ethanol yields milky and soft gels. These experimental results provided motivation to explore the use of both solvents as a mixture. Various volumetric ratios were tested when methanol and ethanol were used, and as described in Figure 5-2, the best quality gels were produced in a milky and rigid form when the volumetric ratio of the two solvents was $50 \%$ $50 \%$. A series of sample aerogels were produced with different target densities and all the 

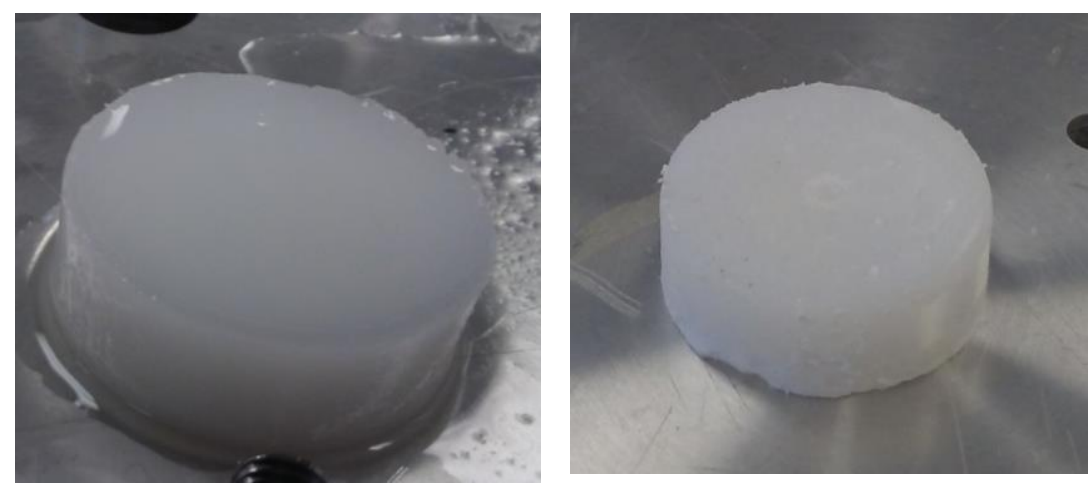

Figure 5-3. Photograph of the synthesized yttria alcogel (left) and aerogel (right).

samples looked opaque before and after supercritical drying as shown in Figure 5-3. The relative molar ratios of $\mathrm{YCl}_{3} \cdot 6 \mathrm{H}_{2} \mathrm{O}: \mathrm{H}_{2} \mathrm{O}: \mathrm{C}_{3} \mathrm{H}_{6} \mathrm{O}$ were $1.0: 6.1: 4.3$, and the methanol and ethanol were adjusted in volumes according to the target densities so the relative molar ratios were in the range of $14.9 \sim 20.9$ and $10.4 \sim 14.6$ for 1 mole of $\mathrm{YCl}_{3} \cdot 6 \mathrm{H}_{2} \mathrm{O}$, respectively.

Table 5-2 illustrates the resulting gelation times, target densities, and measured densities of the produced yttria aerogel monoliths. The gelation time was steadily increased when the target density was decreased. While there were certain differences between the apparent densities and target densities of each aerogel due to the syneresis over the aging time, the magnitude of the differences was increased as the target density was decreased so lighter aerogels were produced (from 0.026 to $0.029 \mathrm{~g} / \mathrm{cm}^{3}$ ).

\subsubsection{Effects of densities on porosity}

Figure 5-4 shows the porosity trends with the different densities of yttria aerogels. The apparent density was observed to be inversely proportional to the change in the estimated 
porosity. On the other hand, porosity was observed to increase with increasing skeletal density due to the fact that the lower target density provides them with more space where the crosslinking occur, leading towards more multidemensional direction during the formation of

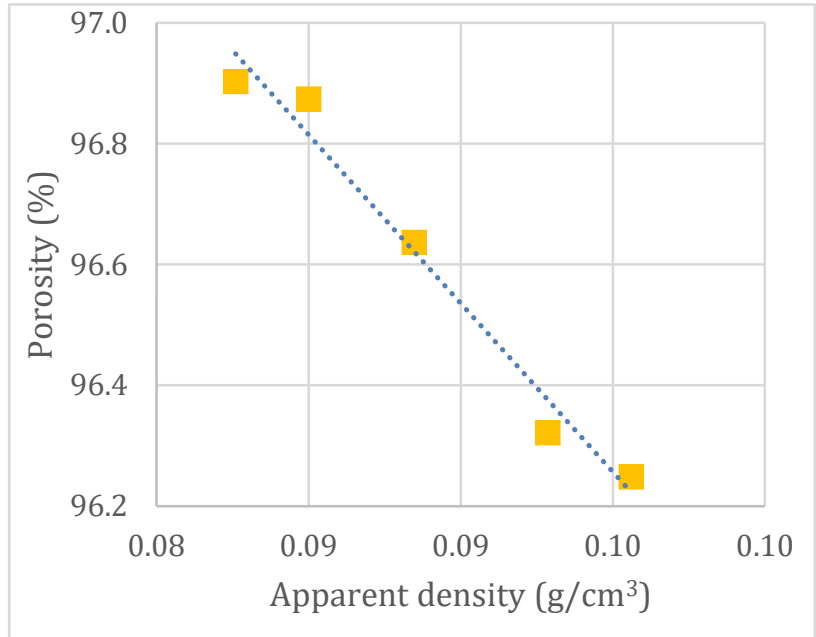

(a)

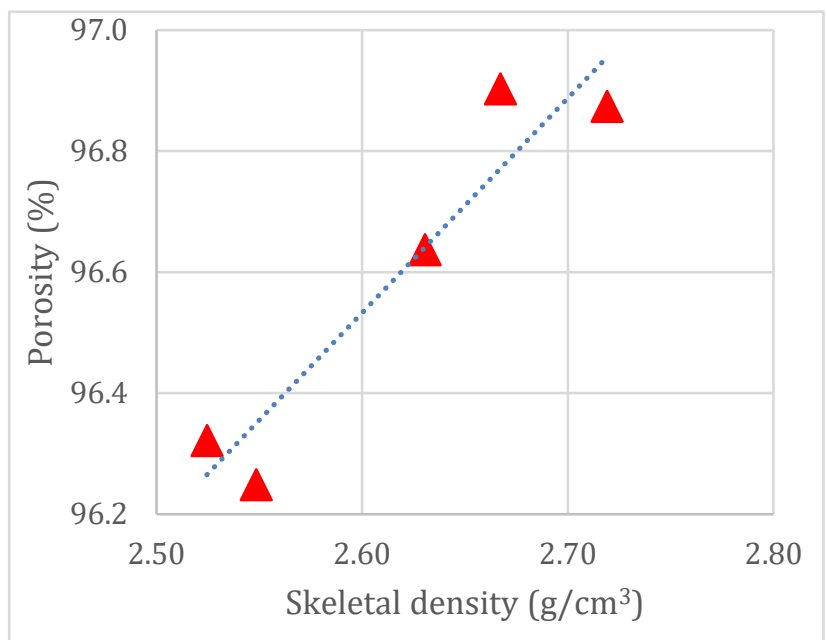

(b)

Figure 5-4. Change of the porosity values with the apparent densities (a) and the skeletal densities (b) of the monolithic yttria aerogels. 
the yttrium oxide networks by poly condensation in the gelation step compared to the synthetic condition with higher target density.

\subsubsection{Pore size distribution and textural properties by $\mathrm{N}_{2}$ adsorption- desorption}

As illustrated in Figure 5-5, the pore size distributions of the produced yttria aerogels are remarkably different in peak shape and range of pore volume from those of the silica aerogel produced in this dissertation. They showed a main pore size region at around $20 \sim 30 \mathrm{~nm}$ and some irregular distrubutions under $10 \mathrm{~nm}$ pore size. Although they seem to have untypical pore structures as aerogels, a trend was observed in change of the pore size distributions as the target density was changed. The pore size distribution was more simplified and narrowed slightly at the higher target density. On the other hand, such results can be the basis for the further study on optimizing the porous structures of yttria aerogels by re-adjusting the molar ratios between the reactants as it has been reported that the surface area of the yttria aerogel is greatly affected

Table 5-3. Effects of target density on textural properties of monolithic yttria aerogels.

\begin{tabular}{lccc}
\hline \hline $\begin{array}{l}\text { target density } \\
\text { of yttria aerogel } \\
\left(\mathrm{g} / \mathrm{cm}^{3}\right)\end{array}$ & $\begin{array}{c}\text { BET surface area } \\
\left(\mathrm{m}^{2} / \mathrm{g}\right)\end{array}$ & $\begin{array}{c}\text { pore volume } \\
\left(\mathrm{N}_{2} \text { desorption }\right) \\
(\mathrm{cc} / \mathrm{g})\end{array}$ & $\begin{array}{c}\text { avg. pore } \\
\text { diameter } \\
(\mathrm{nm})\end{array}$ \\
\hline 0.053 & 75.9 & 0.117 & 6.62 \\
0.057 & 43.5 & 0.082 & 6.65 \\
0.058 & 115.6 & 0.193 & 5.91 \\
0.065 & 63.6 & 0.088 & 6.14 \\
0.069 & 46.8 & & 6.24 \\
\hline \hline
\end{tabular}


by the quantification of propylene oxide for example [121]. Table 5-3 lists the characterization results from the BET and the $\mathrm{BJH}$ methods along with the target density change.

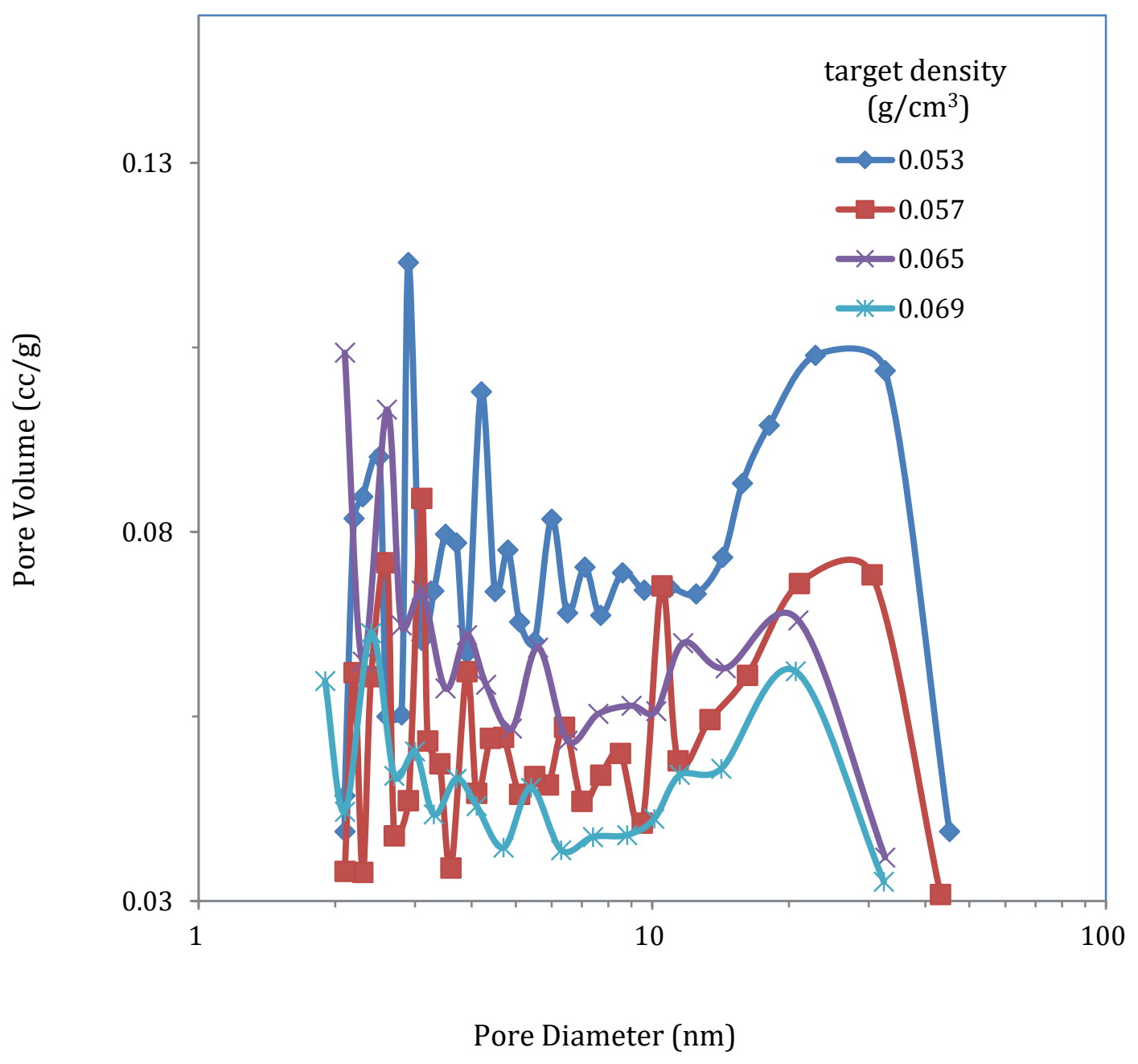

Figure 5-5. Effects of the target density change on the pore size distribution of the produced yttria aerogels. 


\subsection{Synthesis and porosity study of organic}

\section{aerogel}

Organic aerogel was first introduced by Pekala et al. as aqueous, sol-gel polymerization of resorcinol (1, 3 dihydroxy benzene) with formaldehyde [122] and it has been actively investigated because pyrolysis in an inert atmosphere leads to pure carbon aerogels [123]. While the synthetic methods have been diversified, the resulting organic aerogel and carbon aerogel have been nominated for hydrogen and electrical energy storage [124], catalytic support for the

Table 5-4. List of reagents used for the organic aerogel processing

\begin{tabular}{|c|c|c|c|c|c|}
\hline Reagent & Formula & Mol. wt. & $\begin{array}{l}\text { Density } \\
\left(\mathrm{g} / \mathrm{cm}^{3}\right)\end{array}$ & $\begin{array}{c}\text { Boiling Point } \\
\left({ }^{\circ} \mathrm{C}\right)\end{array}$ & Supplier \\
\hline Phloroglucinol & $\mathrm{C}_{6} \mathrm{H}_{6} \mathrm{O}_{3}$ & 126.11 & N/A & $\begin{array}{c}215^{\sim 220} \\
\text { (melting point) }\end{array}$ & Aldrich \\
\hline Formaldehyde & $\mathrm{CH}_{2} \mathrm{O}$ & 30.03 & 1.09 & $\begin{array}{c}100 \\
(37 \% \text { conc.) }\end{array}$ & Aldrich \\
\hline Ethanol & $\mathrm{C}_{2} \mathrm{H}_{6} \mathrm{O}$ & 46.07 & 0.79 & 78.4 & Aldrich \\
\hline $\begin{array}{l}\text { Ammonia } \\
\text { solution }\end{array}$ & $\mathrm{NH}_{4} \mathrm{OH}$ & 40.00 & 0.504 & 36.0 & $\begin{array}{l}\text { Cole- } \\
\text { Palmer }\end{array}$ \\
\hline
\end{tabular}


platinum catalyst [125], and more recently as a high surface container for various inorganic fillers such as clay, metal oxides or CNT/graphene $[126,127,128,129,130,131,132,133]$.

A combination of phloroglucinol (1,3,5-trihydroxybenzene) and formaldehyde was employed to prepare an organic wet gel and aerogel monolith (PF aerogel). Conventionally, resorcinol has been widely used to synthesize organic gel structures but it needs the extra solvent exchange step to remove the water in the solvent which is time-consuming. Meanwhile, phloroglucinol has a faster reaction rate of polycondensation thanks to its enhanced electron density in the 2, 4, 6 ring position. It also offers the opportunity for a wider range of target densities as the gelation can effectively occurs even at higher volumetric ratios of the solvent to the precursor, thus ultralight organic and carbon aerogel can be produced. [125].

\subsubsection{Experimental method}

Phloroglucinol and formaldehyde were dissolved in ethanol and vigorously stirred for 15 minutes and ammonia was added to the PF solution after it became yellowish, which is a sign of complete dissolution. The catalyzed PF solution was additionally stirred for several hours to drive the condensation reaction enough at room temperature because it results in sufficient syneresis after gelation occurs which is necessary to separate the synthesized gel from the casting mold. Gelation was finalized in the convection oven at $338 \mathrm{~K}\left(65^{\circ} \mathrm{C}\right)$ for $30 \sim 120$ minutes depending upon the target density. The relative molar ratios of phloroglucinol : formaldehyde : ammonia (base catalyst) were 1.00:2.13:0.06, and the ethanol was adjusted in volumes according to the target densities so the relative molar ratios were in the range of 12.97 34.60. Table 5-4 lists the chemical reagents used for this research. The synthesized alcogels were placed in the autoclave and transformed to aerogels via the COLD method of the supercritical dying process as introduced in Chapter 2. Five different target densities were 
selected to produce a range of organic aerogel monoliths and they were analyzed by BET, BJH, and density measurements using the helium pycnometer.

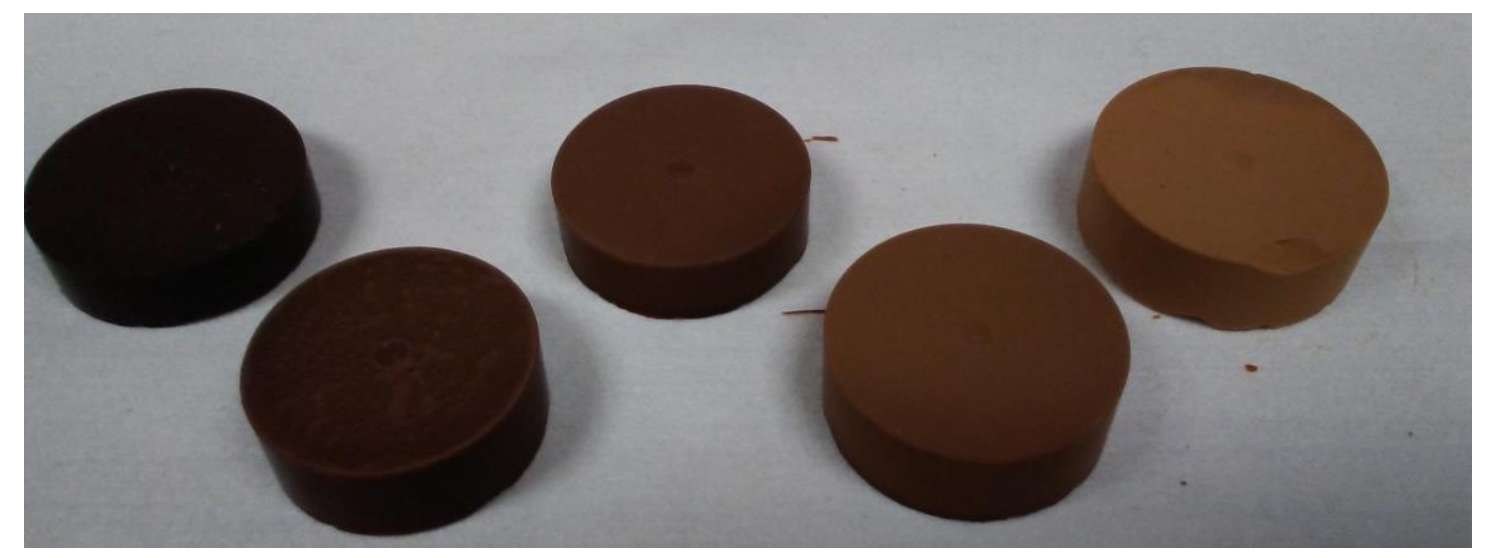

Figure 5-6. Photograph of the produced organic aerogel monoliths. Note that the color differences between the samples come from the different apparent densities. From left to right, the apparent density of each aerogel is $0.237,0.209,0.130,0.113$, and $0.087 \mathrm{~g} / \mathrm{cm}^{3}$, respectively.

\subsubsection{Results and discussions}

Figure 5-6 shows the produced monolithic organic aerogels, arranged by order of the apparent density. It is interesting to see that the color of each sample is lighter (or darker) with lower (or higher) apparent density. Such morphological differences can simply be an observation point to relatively and quickly determine the bulk density of the organic aerogel.

While the PF gel can be prepared from an acid catalyst, a base catalyst, or acid-base two step catalysis, base catalysis was employed in this dissertation research utilizing ammonia as the 


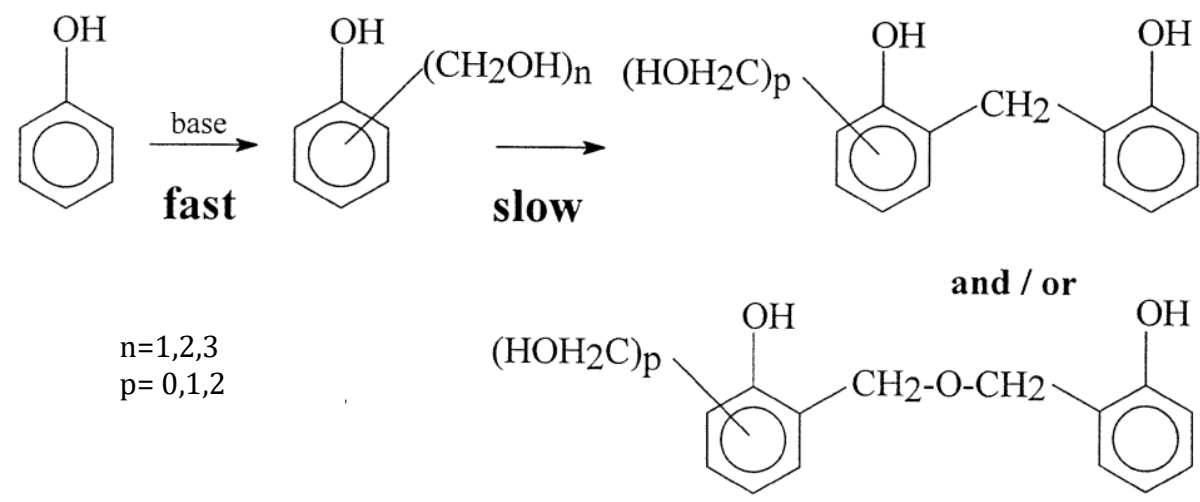

Figure 5-7. Stages of formation of resole resin (base-catalyzed phenolformaldehyde resin) for the base-catalyzed PF gel synthesis. The image was taken from Reference [134].

catalyst. Under basic conditions, formaldehyde reacts quickly with phenolates to form methylol $(-\mathrm{CH}-\mathrm{OH})$ groups. The condensation between a methylol group and a reactive and unsubstituted position of the phenolic ring and between two methylol groups slowly gives rise to methylene $\left(-\mathrm{CH}_{2}-\right)$ and / or ether $\left(-\mathrm{CH}_{2}-\mathrm{O}-\mathrm{CH}_{2}-\right)$ linkages as illustrated in Figure 5-7 [134]. The formation of ether bridges depends on temperature, $\mathrm{pH}$, catalyst type and phenol : formaldehyde ratio [135].

Table 5-5 shows the resulting gelation times, target densities, and measured densities of the produced organic aerogel monoliths. The change in the gelation time was observed to have a similar influence to that observed in silica and in yttria aerogel, with increased gelation times resulting when the target density was decreased. The magnitude of the difference between the target density and the apparent density was considerably decreased as the target density was decreased, unlike that observed in silica or yttria aerogels. This provides evidence that the syneresis at lower target density is less active due to the slow condensation in the base catalyzed 
system, which preserves the inherent structure and volume of the alcogel more during the transformation to an aerogel.

Table 5-5. Resulting gelation time and corresponding densities of the monolithic organic aerogels.

\begin{tabular}{lcccc}
\hline \hline Sample ID & $\begin{array}{c}\text { gelation time } \\
\left(\text { at } 65^{\circ} \mathrm{C}, \mathrm{min}\right)\end{array}$ & $\begin{array}{c}\text { target density } \\
\left(\mathrm{g} / \mathrm{cm}^{3}\right)\end{array}$ & $\begin{array}{c}\text { apparent } \\
\text { density }\left(\mathrm{g} / \mathrm{cm}^{3}\right)\end{array}$ & $\begin{array}{c}\text { skeleton } \\
\text { density }\left(\mathrm{g} / \mathrm{cm}^{3}\right)\end{array}$ \\
\hline \hline OA15 & 30 & 0.186 & 0.237 & 1.537 \\
OA20 & 40 & 0.149 & 0.209 & 1.522 \\
OA25 & 60 & 0.124 & 0.130 & 1.533 \\
OA30 & 80 & 0.106 & 0.113 & 1.536 \\
OA40 & 120 & 0.083 & 0.087 & 1.601 \\
\hline \hline
\end{tabular}

\subsubsection{Effects of densities on porosity}

Figure 5-8 shows the porosity trends with the different densities of the organic aerogels. The apparent density was inversely proportional to the change in estimated porosity. The change in skeletal density was less clear, but generally the porosity was sharply increased with small increases in the skeletal density (there was one exception). This unusual sensitivity suggests the possibility that the sol-gel process can be designed to control the skeletal density with minimum change in the apparent density, allowing the resulting porosity to be intentionally adjusted. As organic aerogels have been utilized as thermal insulation in high temperature environments, this 
result suggests further research on development of a modified production process to tune the porosity of organic aerogels.

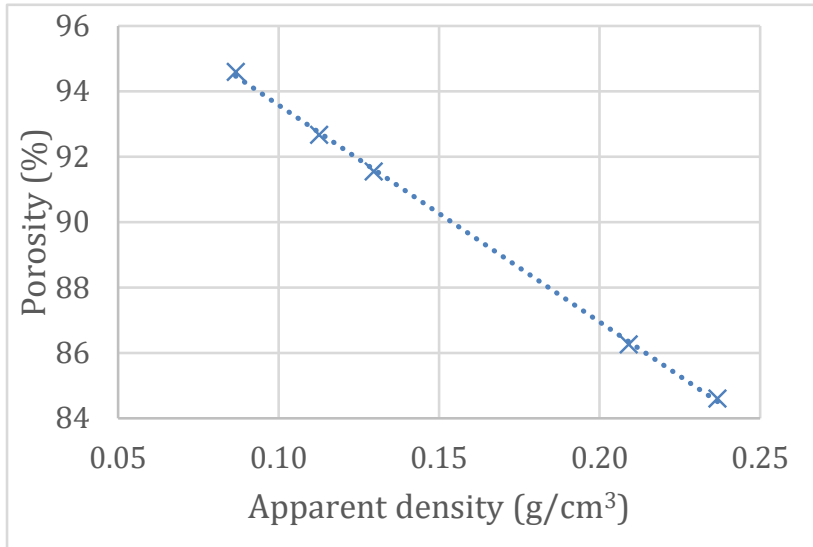

(a)

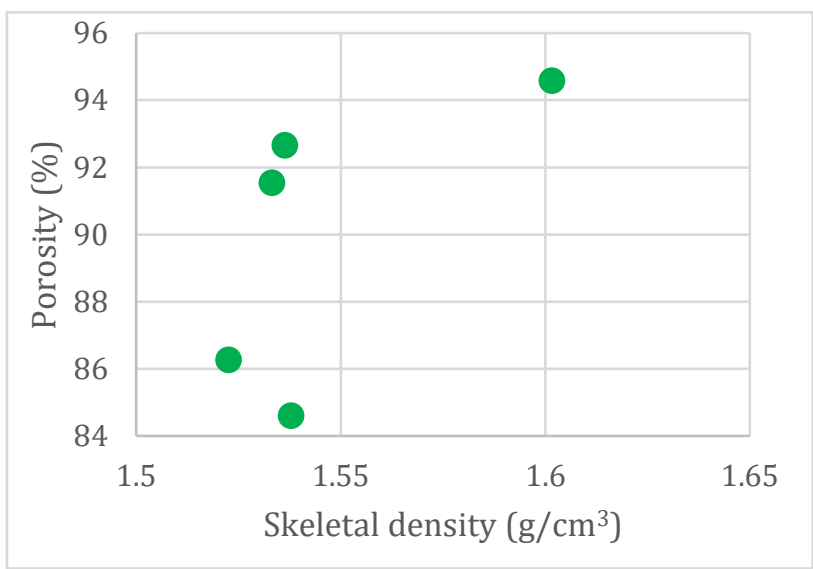

(b)

Figure 5-8. Change of the porosity values with the apparent densities (a) and the skeletal densities (b) of the monolithic organic aerogels. 


\subsubsection{Pore size distribution and textural properties by $\mathrm{N}_{2}$ adsorption- desorption}

Figure 5-9 shows the well-developed pore size distributions of the produced organic aerogels and the clear trend of shifting the peak of pore size distribution to larger values with increased target density. As the distibution shifts to larger values with increased target density, the width of the distribution is reduced as well. This implies that higher target density results in smaller space for the organic groups to form gel networks so the variations in their shape and the sizes of the pores created between them are smaller than those resulting in lower target density gels. From this result, it can be expected that thermal transport in organic aerogels will

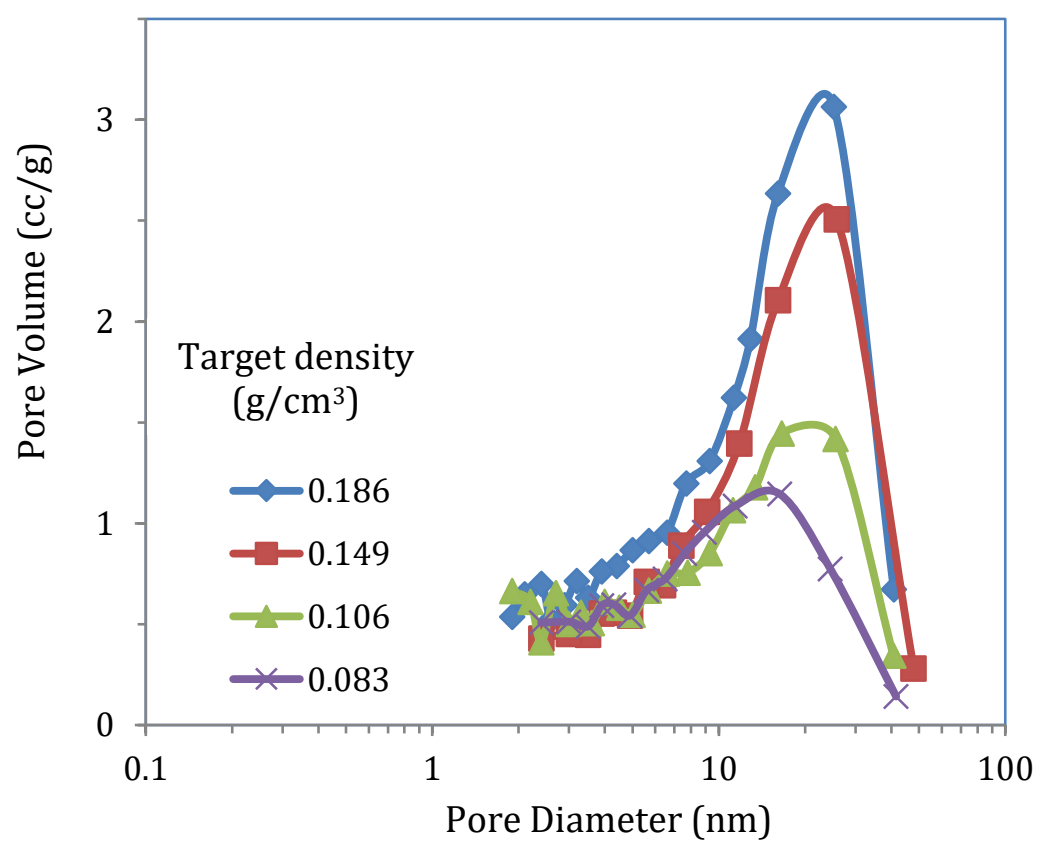

Figure 5-9. Effects of the target density change on the pore size distribution of the produced organic aerogels. 
Table 5-6. Effects of target density on textural properties of monolithic organic aerogels.

\begin{tabular}{lccc}
\hline $\begin{array}{l}\text { target density } \\
\text { of organic aerogel } \\
\left(\mathrm{g} / \mathrm{cm}^{3}\right)\end{array}$ & $\begin{array}{c}\text { BET surface area } \\
\left(\mathrm{m}^{2} / \mathrm{g}\right)\end{array}$ & $\begin{array}{c}\text { pore volume } \\
\left(\mathrm{N}_{2} \text { desorption) }\right. \\
(\mathbf{c c} / \mathrm{g})\end{array}$ & $\begin{array}{c}\text { avg. pore } \\
\text { diameter } \\
(\mathbf{n m})\end{array}$ \\
\hline 0.083 & 586.6 & 1.01 & 7.88 \\
0.106 & 581.6 & 1.28 & 8.22 \\
0.149 & 664.2 & 1.77 & 11.22 \\
0.186 & & & \\
\hline \hline
\end{tabular}

rely more on the cross linked solid of the organic network because the porosity is decreased with increasing skeletal density at a higher target density as reviewed from the effective medium approximation in Chapter 2.

Table 5-6 lists the characterization results from the BET and BJH methods along with the target density change. The surface areas, pore volumes, and average pore diameters were all increased when the target density was increased. These results show that the gel networks were built up more homogenously during the condensation step at higher target density where the reaction is more active. On the other hand, these results disagree with what has been conventionally understood for the aerogel structure. That is, high porosity is obtained from the high homogeniety of the porous structue. Further study can pursue this unusual pattern, as there has been no prior work reported on the porosity of organic aerogel. 


\subsection{Conclusions}

Monolithic yttria aerogels were produced by using yttrium chloride (III) hexahydrate as a precursor and propylene oxide as an epoxide that plays a role as the proton scavenger to enhance the sol-gel reaction. The methanol - ethanol mixture at $50 \mathrm{vol} \%$ ratio was found to contribute to producing a rigid monolith of yttria alcogel at ambient temperature with approximately 40 vol\% variability where the target density can be adjusted up to $25 \%$ in a relative value. The porosity of the yttria aerogels showed an inversely proportional relationship to the apparent density, whereas the skeletal density showed a proportional trend to the porosity as they both increased with decreased target density. This result suggests a direction for a synthetic strategy in order to obtain a specific skeletal density. The relative molar ratio of the propylene oxide is considered to be the next parameter to be investigated, as it critically affects the structural property of the produced yttria aerogel.

Organic aerogel monoliths were produced by using a combination of phloroglucinol formaldehyde as a precursor and ammonia as a polymerization (gelation) catalyst. The selected synthetic route enabled several target densities of the synthetic conditions producing crack-free monolithic aerogels repeatedly at $338 \mathrm{~K}\left(65^{\circ} \mathrm{C}\right)$ within 2 hours. The produced aerogels showed well developed pore size distributions with a clearly ordered difference between the different target densities. These different target densities also resulted in different apparent densities as well as different skeletal densities. From the porosimetry analysis, using the nitrogen adsorption - desorption method, the BET surface area, the BJH pore volume, and the average pore diameter were steadily increased, which implies that the homogeneity of the pore formations is improved with the increased target density. The porosity showed high sensitivity to the apparent density in the organic aerogel, so it was rapidly increased when apparent density was decreased. The skeletal density remained at a relatively constant value with change in porosity within the 
studied range. These results aided in defining the range of target density for future research on organic aerogel in order to understand the porosity change as well as the porosity. 


\section{Chapter 6}

\section{Thermal Conductivity of Monolithic Aerogel}

\section{Determined with Implanted Wire Set by $3 \omega$}

\section{Method}

This chapter presents results demonstrating use of an implanted wire set with the $3 \oplus$ method for measuring thermal conductivity of monolithic aerogel. The chapter very briefly reviews the theoretical background for the experimental setup and provides details of the implementation of the system used in this demonstration. A customized sample preparation method has been developed and is used to measure thermal conductivity of representative monolithic aerogels. It is demonstrated that this new method of sample preparation yields reliable sample data that support the dissertation claims of lower thermal conductivity with higher porosity among the measured samples.

\subsection{Review of the $3 \omega$ Method}

The thermal properties of the produced sample aerogels have been measured using the $3 \varpi$ thermal conductivity measurement setup in the UVa Nanoscale Energy Transfer Lab. The $3 \varpi$ method has been used extensively to measure the thermal properties of bulk and thin film dielectric materials $[136,84,137,138,139]$. The method employs a metallic strip or a thin metal 
wire in intimate contact with the measured sample. In this research, the aerogel samples have been produced as monoliths, and the thin metal wire set was installed inside each sample.

During the measurement, an AC electrical current modulated at angular frequency $\omega$ is induced to flow in the wire causing heat generation in it. The heating has both a DC component, which changes the average temperature of the sample, and an $\mathrm{AC}$ component at $2 \omega$, which generates thermal waves in the sample. Because the electrical resistance of the wire depends on the temperature, the resistance is modulated at $2 \omega$ as well. Therefore, an AC voltage drop is experienced across the ends of the wire at $3 \omega, V_{3 \omega}$, which is proportional to the AC temperature variation of the wire at $2 \omega$, referred to $T_{2 \omega}$. $T_{2 \omega}$ will depend on the thermal conductivity, $k$, of the monolithic aerogel where this wire set is implanted. Thus, it is possible to extract $k$ from a measurement of $V_{3 \omega}$ vs. $\omega$.

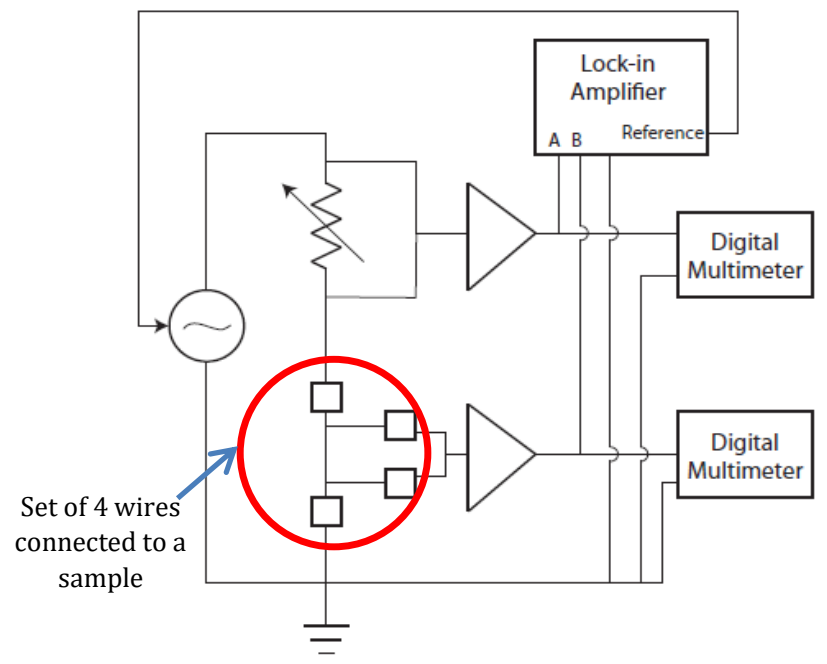

Figure 6-1. Depiction of simplified 3w. Modified from the reference [140]. The monolithic aerogel sample is connected to the 4 -wire set by the 4 wires implanted in the sample. 
The circuit design used in this work is depicted in Figure 6-1. Current passes from the lockin amplifier through the potentiometer, through the sample heating wire, and is then grounded. Two digital multimeters and the lock-in amplifier are connected to each element through a differential amplifier. The multimeters are used to determine the first harmonic voltage drop across each element. These readings are used to determine the sample's electrical resistance (and therefore the amount of heat deposited into the sample). The A and B inputs on the lock-in amplifier read the third harmonic voltage drop across each element. The difference in these values is calculated and used to determine the second harmonic temperature rise in the heating wire and ultimately the sample's thermal conductivity [140].

\subsection{Customized Sample Preparation for $3 \varpi$}

\section{Measurement}

Conventionally, the $3 \curvearrowleft$ method requires sample post processing to install heating and sensing elements on the desired sample. This would require thin metallic strips be deposited onto the aerogel sample surface in a pre-determined configuration. The post metal processing must be completed separately from the synthetic process for aerogel itself, and most often would occur in a clean-room. I have demonstrated the ability to eliminate this post processing by merging the installment step of the heating and sensing media with the aerogel synthesis process. The sample preparation procedure for the $3 \bowtie$ sample is as follows:

1. A wire set for $3 \varpi$ measurement is designed and fabricated in advance as visually described in Figure 6-2. Nickel-chrome alloy wire is used as the heat source and the voltage reader. This heating wire is connected at both ends to a pair of copper wires. 
2. A sol-to-gel solution prepared by the sol-gel route is transferred to a cylindrical

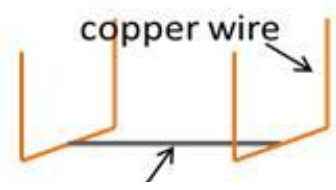

nickel-chrome wire
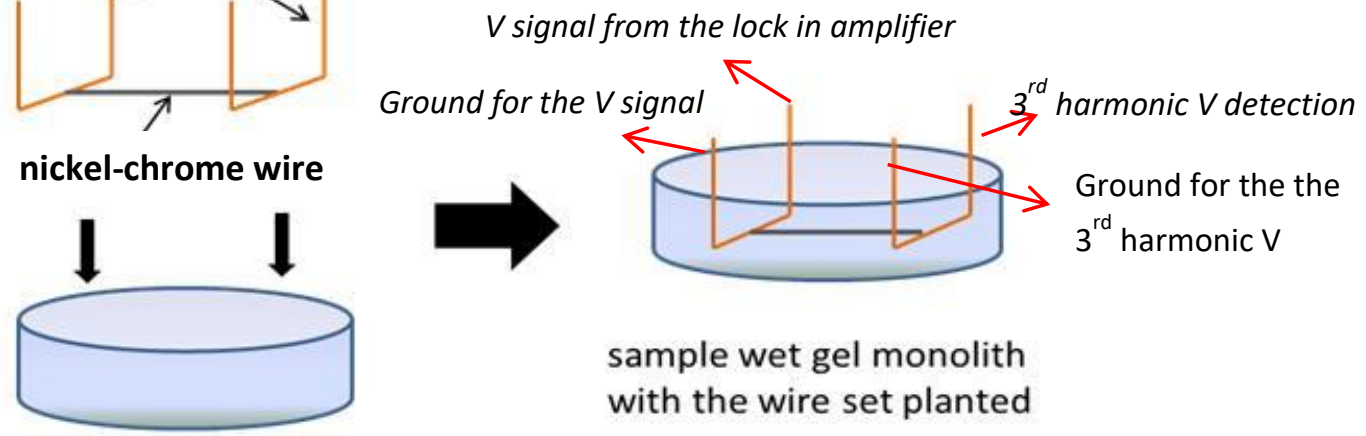

sample wet gel monolith

with the wire set planted

monolithic wet gel (soft)

Figure 6-2. Schematic diagram of procedure for sample aerogel preparation for $3 \omega$ measurement.

container for casting.

3. Gelation of the sol-to-gel solution is confirmed by the tilting method at around the estimated gel time.

4. Once gelation starts, the wire set is slowly introduced from the top surface of the soft gel until the wire set is positioned at the middle point of the gel body.

5. The container is closed and the gel ages with the "normal" aerogel production process continuing in order to produce a sample aerogel for $3 \omega$ measurement.

Once the sample aerogel is produced, the four ends of the copper wires protruding from the aerogel are connected to the $3 \varpi$ setup as shown in Figure 6-3. The voltage signal from the lock-in amplifier is connected to the lower left wire (red arrow). The ground for this signal is 
connected to the lower right wire (blue arrow). The third harmonic voltage detection is connected to the upper left wire (green arrow), and the third harmonic voltage detection ground is connected to the upper right wire (black arrow).

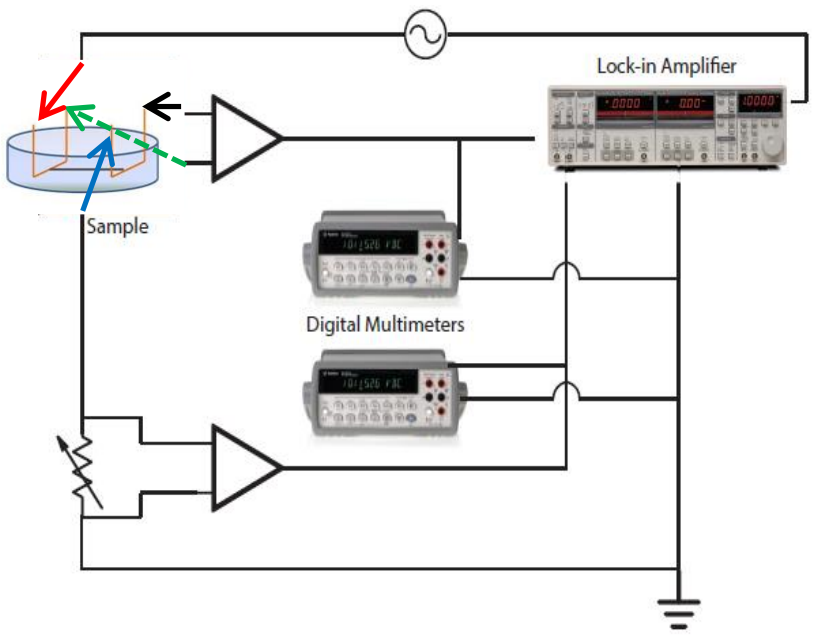

Figure 6-3. Schematic diagram of the 3-omega set and the connected monolithic aerogel sample with the implanted wire set (Modified from the reference [140]).

The $3 \oplus$ experimental measurement procedure is then the same as that for a thin film sample repeated numerous times with this facility. The change in resistance with temperature, $d R / d T$ of the heated wire (nickel-chrome alloy) can be obtained by calibrating the temperature increase of the wire with time as it is heated. The heat diffusion equation for the produced sample has been modified by considering the heat source geometry. 


\subsection{Determining a Heat Diffusion Equation}

This section describes the methodology reported from references used to obtain thermal conductivity from the $3 \omega$ method $[141,142,143]$. The $3 \omega$ voltage component, $V_{3 \omega}$ is related to the temperature oscillation, $\Delta T$, by:

$$
\Delta T=2 \frac{d T}{d R} \frac{R_{0}}{V} V_{3 \omega}
$$

where $V$ is the applied voltage and $R_{0}$ is the electrical resistance along the metal wire. The solution to the heat equation in the wire geometry proceeds according to the cylindrical heat source model provided by Carslaw and Jaeger [144]. Birge's implementation of this solution is used throughout this work to solve for thermal conductivity of the monolithic aerogel with the implanted wire set [142]. Assuming that the heating wire is infinitely long, and its radius is very small $(63 \mu \mathrm{m})$ compared to the thermal wavelength (calculated to be $3200 \mu \mathrm{m}$ at the averaged frequency used for the measurement, $365 \mathrm{~Hz}$ ), the $\mathrm{AC}$ temperature rise average over the heating wire is

$$
\Delta T=\frac{P}{2 \pi l k}\left(\ln (\omega)^{\frac{1}{2}}-\gamma\right)
$$

where $P$ is the input power, $l$ is the heater length, $\gamma$ is Euler's constant $(0.5772 \ldots)$, and $k$ is the thermal conductivity of a material of interest surrounding the heating wire [142]. Equation (6.2) shows that $k$ is frequency independent and the heating oscillation only depends on $k$, which is the thermal conductivity of the produced aerogel monolith in this research. 


\subsection{Sample Preparation and Anticipated Results}

Silica aerogels and organic aerogels were prepared with the wire sets described in the previous section. Yttria aerogel was also prepared, but excluded as the initial attempt showed that this aerogel is more brittle due to its low apparent density (equal or less than $0.1 \mathrm{~g} / \mathrm{cm}^{3}$ ). This initial result led to need for further investigation in order to connect the implanted wire set to the sensor wires of the $3 \omega$ system without damaging the sample. This section describes the aerogel samples produced for these $3 \omega$ measurements and anticipated results, based on the theory and discussions, which have been presented in this dissertation.

\subsubsection{Preparation of silica aerogels with wire sets}

In Chapter 4, I introduced 9 samples of monolithic silica aerogels which were produced with 9 different target densities. Out of these 9 samples, 3 samples, whose ID's are 3.0/7.0, 4.5/5.5, and 5.0/5.0, were reproduced with the wire sets implanted inside them. These 3 samples were synthesized by the DCM (Direct-contact method), my newly developed two step sol-gel process. The nitric acid-acetic acid combination was used for pre-hydrolysis catalyst. After prehydrolyzed silica sol was prepared, adjusted amounts of this sol, the solvent (ethanol), and the gelation catalyst (ammonia) were vigorously mixed according to the designated target densities. The target densities are $0.0582 \mathrm{~g} / \mathrm{cm}^{3}$ for sample ID $3.0 / 7.0,0.0873 \mathrm{~g} / \mathrm{cm}^{3}$ for $4.5 / 5.5$, and 0.0970 $\mathrm{g} / \mathrm{cm}^{3}$ for $5.0 / 5.0$.

As gelation times of these 3 samples were known from the investigation results in Chapter 4, it was possible to implant the wire set 1 minute before the predicted gelation occurred. The viscosity of the sol-to-gel solution rapidly increased at this time, so the implanted wire set sank 
very slowly when it was gently dropped on the top surface of this sol-to-gel solution. As the gelation was completed, the implanted wire set was held in the middle of the alcogel monolith. The four ends of the copper wires protruded from the top surface of the gel. A brief description of the synthetic conditions of these selected aerogels is listed in Table 6-1.

Table 6-1. List of wire set-implanted silica aerogels prepared with the wire sets for the $3 \curvearrowleft$ measurement for thermal conductivity.

\begin{tabular}{llccc}
\hline Sample ID & Production process & $\begin{array}{c}\text { Target density } \\
\left(\mathbf{g} / \mathbf{c m}^{3}\right)\end{array}$ & $\begin{array}{c}\text { Gelation time } \\
\text { (minutes) }\end{array}$ & $\begin{array}{c}\text { Wire set } \\
\text { implantation } \\
\text { time } \\
\text { (minutes) }\end{array}$ \\
\hline $3.0 / 7.0$ & $\begin{array}{l}0.0582 \\
\text { The DCM (see Chapter 3) as } \\
\text { sol-gel process followed by } \mathrm{CO}_{2} \\
\text { supercritical drying. }\end{array}$ & 0.0873 & 19 & 18 \\
$5.0 / 5.0$ & & 0.0970 & 18 & 17 \\
\hline
\end{tabular}

Porosities of the produced silica aerogels are $87.295 \%$ for sample ID 5.0/5.0, 89.660\% for 4.5/5.5, and $93.061 \%$ for 3.0/7.0. As discussed in Chapter 2, the relative value of thermal conductivity of silica aerogel is inversely proportional to the porosity. Therefore, thermal conductivity of 5.0/5.0 is expected to be the highest and that of 3.0/7.0 will be the lowest of the three. Razaei et al. reported a thermal conductivity of $13.4 \mathrm{~mW} / \mathrm{m} \cdot \mathrm{K}$ at $303 \mathrm{~K}\left(30^{\circ} \mathrm{C}\right)$ for silica aerogel produced from sodium silicate with an apparent density of $0.140 \mathrm{~g} / \mathrm{cm}^{3}$ [145]. Caps et al. reported a value of $18.0 \mathrm{~mW} / \mathrm{m} \cdot \mathrm{K}$ measured at ambient temperature for a monolithic transparent silica aerogel without specifying density of their sample [146]. Cohen et al. measured $9.3 \mathrm{~mW} / \mathrm{m} \cdot \mathrm{K}$ from their silica aerogel prepared by a modified two step sol-gel method as they assumed its porosity to be $93.6 \%$ [147]. Based on these available references, the 
anticipated range of thermal conductivity in this research is as low as $9.0 \mathrm{~mW} / \mathrm{m} \cdot \mathrm{K}$ to 20.0 $\mathrm{mW} / \mathrm{m} \cdot \mathrm{K}$ as a maximum value.

\subsubsection{Preparation of organic aerogels with wire sets}

Two organic aerogels were selected and reproduced for $3 \omega$ measurements from the 5 gels introduced and investigated in Chapter 5. The precursors were phloroglucinol and formaldehyde and they were first dissolved in ethanol. The volume of ethanol was quantified based on the target densities: $0.1060 \mathrm{~g} / \mathrm{cm}^{3}$ for sample ID OA30 and $0.1861 \mathrm{~g} / \mathrm{cm}^{3}$ for OA15. After stirring this solution for 10 minutes for complete dissolution, an adjusted amount of ammonia was applied to this precursor solution to promote polymerization.

Table 6-2. List of wire set-implanted organic aerogels prepared with the wire sets for the $3 \varpi$ measurement of thermal conductivity.

\begin{tabular}{ccccc}
\hline Sample ID & Production process & $\begin{array}{c}\text { Target density } \\
\left(\mathbf{g} / \mathrm{cm}^{3}\right)\end{array}$ & $\begin{array}{c}\text { Gelation time } \\
\text { (at 338K, min) }\end{array}$ & $\begin{array}{c}\text { Wire set } \\
\text { implantation } \\
\text { time } \\
\text { (minutes) }\end{array}$ \\
\hline OA30 & $\begin{array}{c}\text { Polymerization of } \\
\text { phloroglucinol- } \\
\text { formaldehyde by } \\
\text { ammonia at } 65^{\circ} \mathrm{C},\end{array}$ & 0.1060 & 80 & 77 \\
\hline OA15 & $\begin{array}{c}\text { followed by } \mathrm{CO}_{2} \\
\text { supercritical drying. }\end{array}$ & 0.1861 & 30 & 27 \\
\hline
\end{tabular}

Unlike the silica aerogel, the sol-to-gel solution for the organic wet gel was placed in a convection oven to be heated at $338 \mathrm{~K}\left(65^{\circ} \mathrm{C}\right)$ for gelation. When the gelation time was about to occur and the viscosity was increased, the organic sol-to-gel solution was taken out of the oven for the wire set implantation. After the wire set was implanted, the sol-to-gel solution was placed 
back in the oven, and stored there until the gelation was completed. Table 6-2 shows a brief description on the synthetic conditions of the produced organic aerogels.

Porosities of these samples are $84.608 \%$ for OA15 and $92.670 \%$ for OA30. Similar to the prediction for the silica aerogels in this study, thermal conductivity of OA30 is expected to be lower than that of OA15, as the porosity of OA30 is higher. Lu et al. reported thermal conductivities of organic aerogels prepared from resorcinol-formaldehyde between 13 and 19 $\mathrm{mW} / \mathrm{m} \cdot \mathrm{K}$, when apparent densities were between 0.103 and $0.279 \mathrm{~g} / \mathrm{cm}^{3}$ [148]. Based on their report, I anticipate the thermal conductivity of our phloroglucinol-formaldehyde organic aerogels to be from 13 to $20 \mathrm{~mW} / \mathrm{m} \cdot \mathrm{K}$ as their apparent densities are $0.1126(\mathrm{OA} 30)$ and 0.2367 $\mathrm{g} / \mathrm{cm}^{3}(\mathrm{OA} 15)$

\subsection{Results and Brief Review}

The common method for preparing a sample for characterization using the $3 \omega$ technique is fabrication of a thin film strip heater on the substrate material (which can be bulk or a thin film on a substrate) to be investigated [140]. When this conventional sample preparation is used for the $3 \omega$ method, oftentimes the properties of an electrically conductive substrate are of interest and it is then necessary to prepare an electrically insulative layer between the target sample and the heating wire. This more complicated geometry then requires analysis of the sensitivity trends of thermal conductivity, thermal contact resistance, passivation layer thickness, and heating wire width of the measured sample to the input frequency. These studies are necessary to distinguish the properties of the target sample from these other parameters. However, this wire set implanted method eliminates the need for these preliminary studies as the thermal conductivity of the prepared sample is only the parameter of interest. 
In Bauer's previously published work, he reported the input frequency range of $10 \mathrm{~Hz}$ to $10 \mathrm{kHz}$ yielded data with the most sensitivity to the thermal conductivity of the aerogel [140]. Though Bauer was using a thin film aerogel with a strip heater providing an one dimensional heating source, in this work it has been assumed the same frequency range will yield the best results for these monolithic aerogel samples with the implanted wire sets providing a cylindrical heating source. This is an assumption that should be examined in later work if detailed studies of the resulting thermal conductivity are conducted.

Table 6-3. Physical properties and measurement results of $3 \varpi$ for monolithic aerogels with implanted wire sets.

\begin{tabular}{lcccc}
\hline Sample ID & $\begin{array}{c}\text { Apparent density } \\
\left(\mathrm{g} / \mathrm{cm}^{3}\right)\end{array}$ & $\begin{array}{c}\text { Skeletal density } \\
\left(\mathrm{g} / \mathrm{cm}^{3}\right)\end{array}$ & Porosity (\%) & $\begin{array}{c}\text { Thermal } \\
\text { conductivity } \\
(\mathrm{mW} / \mathrm{m} \cdot \mathrm{K})\end{array}$ \\
\hline $\mathbf{3}$ Silica aerogel & 0.1285 & 1.8521 & 93.061 & $14.4 \pm 1.2$ \\
$\mathbf{4 . 5 / 5 . 5}$ & 0.1922 & 1.8591 & 89.660 & $15.7 \pm 1.4$ \\
$\mathbf{5 . 0 / 5 . 0}$ & 0.2188 & 1.7226 & 87.295 & $17.5 \pm 1.5$ \\
Organic aerogel & & & & \\
\hline OA30 & & & & \\
\hline OA15 & 0.1126 & 1.5364 & 92.670 & $15.5 \pm 1.4$ \\
\hline
\end{tabular}

Table 6-3 shows the experimental results of the $3 \omega$ measurements on the produced aerogels. The overall trend of all data shows good agreement with what have been discussed in this dissertation. Thermal conductivities of both silica and organic aerogels were increased with 
decreasing porosities. A 9.0\% uncertainty in the data is assumed, in accordance with Bauer's research previously pursed utilizing the same $3 \omega$ apparatus in the UVa Nanoscale Energy Transfer Lab [140]. As predicted in Section 6.4, thermal conductivity of the silica aerogels obtained from this method are in the range of $9.0-20.0 \mathrm{~mW} / \mathrm{m} \cdot \mathrm{K}$. These results agree well with results reported by Cohen et al. as discussed in this chapter (Section 6.4.1) [147] and Wei et al. that were presented in Chapter 2 (Figure 2-5) [94]. Data obtained from measuring the organic aerogels range from 15.5 and $20.1 \mathrm{~mW} / \mathrm{m} \cdot \mathrm{K}$ and they also show good agreement with predictions discussed in Section 6.4.2 $(13.0-20.0 \mathrm{~mW} / \mathrm{m} \cdot \mathrm{K})[146]$. 


\section{Chapter 7}

\section{Conclusions}

\subsection{Summary of Conclusions}

The size of the aerogel market in the thermal insulation industry has been steadily increasing over time. In order for commercial aerogel insulation to stay competitive, it cannot be overemphasized how important the successful development of an aerogel production process that has high feasibility for controlling thermal conductivity is. Such a process should also be easily integrated with a conventional material process for hybrid aerogel products. In addition, the new process should contribute to reducing the production cost by simplification of the production process and by reducing the quantity of required materials.

To guide development of a new aerogel production process, a good understanding of how thermal conduction occurs in aerogel is crucial. Through the literature review presented in Chapter 2, I demonstrated the sensitivity of thermal conductivity in aerogel materials to the porosity. Reported experimental results were analyzed and this analysis showed that higher porosity results in lower thermal conductivity for aerogels with porosities of $80 \%$ or greater. I also presented an example of how the production process of aerogel insulation materials was selected to obtain the lowest possible thermal conductivity. When thermal conductivities of the produced aerogels from each of the developed processes were compared, the difference between the lowest thermal conductivity from the selected process and the second lowest one from the non-selected process is $0.7 \mathrm{~mW} / \mathrm{m} \cdot \mathrm{K}$. This example demonstrated that the production process 
of aerogel insulation should be continuously optimized even when the optimized process decreases thermal conductivity of the product by less than $1.0 \mathrm{~mW} / \mathrm{m} \cdot \mathrm{K}$.

Motivated by what were discussed in Chapter 2, I developed a new sol-gel process, called the direct-contact method (DCM, US Provisional Patent Application Serial No. 62/486,057 filed April 17, 2017) and introduced it in Chapter 3. This new process replaces the conventional prehydrolysis step in the two step sol-gel process. By employing this method, silica alkoxide and water are catalyzed for hydrolysis without diluting them in a solvent and heating. As a result, clear and pre-hydrolyzed silica sol is produced and directly available for further gelation steps within a few hours at ambient temperature. The DCM also yields a sol with higher concentration of silica than that obtained from a conventional method, so the density range of the final gel product is extended. This advantage increases feasibility of the entire sol-gel process with regards to combining with any existing material processes. In addition, the produced sol has sufficient pot life span which enables different types of gel products to be produced from one time preparation of pre-hydrolyzed sol. Finally, the production costs can be remarkably reduced as the time and energy consumed by the DCM is much less compared to the conventional two step process. With these advantages, the DCM still produces a pre-hydrolyzed sol that has properties compatible to a functional silane, which is commercially available at a much higher price than TEOS.

By taking advantage of the DCM, I investigated how porosity and the porous structure of silica aerogel is affected by the parameters of its production process. A series of monolithic silica aerogels with a range of densities were produced for characterization and the results were introduced and discussed in Chapter 4. The DCM was successfully applied to the two step solgel route and demonstrated high feasibility for tuning the synthetic conditions to obtain a desired target property of the silica aerogel texture. Solvent type at the gelation step, catalyst type for the DCM process, and the aging treatment to establish hydrophobicity in the aerogel's nature 
affected porosity of the produced aerogel as well as other textural properties. From this investigation, it was demonstrated that the desired target porosity of silica aerogel can be obtained by appropriately adjusting the process parameters. This investigation also demonstrated that it is feasible to employ the DCM for silica aerogel production of optimized thermal conductivity materials from minimal trials.

In Chapter 5, I described my study on the effects of target density on porosity of yttria aerogels and organic aerogels. Both types of aerogel monoliths were produced from modified sol-gel processes and characterized in order to outline how target density is related to apparent density and skeletal density. Monolithic yttria aerogels were produced by using yttrium chloride (III) hexahydrate as a precursor and propylene oxide as an epoxide that plays a role as the proton scavenger to enhance the sol-gel reaction. Through a study of modification of the sol-gel process, I found that a mixture of methanol - ethanol at a volume ratio of $50 \%$ to $50 \%$ used as a solvent results in a rigid monolithic yttria aerogel at ambient temperature. The experimental results showed that increasing target density resulted in increased apparent density and decreased skeletal density. Porosity of the yttria aerogels showed an inversely proportional relationship to the apparent density, whereas the skeletal density showed a proportional one to the porosity. This study aided in recognizing the importance of controlling skeletal density of the produced yttria aerogel in order to obtain a desired porosity by strategizing the sol-gel process. The relative molar ratio of the propylene oxide to yttrium precursor is considered to be the next parameter to be explored as it critically affects the structural property of the produced yttria aerogel.

Organic aerogel monoliths were produced by using a combination of phloroglucinol formaldehyde as a precursor and ammonia as a polymerization (gelation) catalyst. The selected synthetic route enabled several target densities of the synthetic conditions producing crack-free monolithic aerogels repeatedly at $338 \mathrm{~K}\left(65^{\circ} \mathrm{C}\right)$ within 2 hours. The produced aerogels showed 
well developed pore size distributions with a clearly ordered difference between the different target densities. These different target densities also resulted in different apparent densities as well as different skeletal densities. From the porosimetry analysis, using the nitrogen adsorption - desorption method, the BET surface area, the BJH pore volume, and the average pore diameter were steadily increased, which implies that the homogeneity of the pore formations is improved with the increased target density. The porosity showed high sensitivity to the apparent density in the organic aerogel, so it was rapidly increased when apparent density was decreased. The skeletal density remained at a relatively constant value with change in porosity within the studied range. These results aided in defining the range of target density for future research on organic aerogel in order to understand the porosity change as well as the porosity.

In Chapter 6, I presented a newly developed experimental method for preparation of aerogel samples for thermal conductivity measurements using the established $3 \varpi$ method. The new method required installation of a wire set inside a monolithic aerogel so that it can be used for the $3 \varpi$ measurement. The wire set was designed to play the role of the 4 connectors needed for the $3 \varpi$ method. I demonstrated successful implantation of the wire set into both monolithic silica aerogel and monolithic organic aerogel. Results were obtained for a representative set of samples using the implanted wire sets and the $3 \omega$ measurement. These representative experimental $3 \omega$ results of thermal conductivity for the produced aerogels provided confirmation that the changes of porosity and thermal conductivity agreed with what were discussed in Chapter 2. The results showed that thermal conductivities decreased with increased porosities, thus agreeing well the discussion and model presented in this research. These results also demonstrated that the wire set implantation can be a reliable method that simplifies sample preparation for $3 \varpi$ measurements in aerogel materials. 


\subsection{Suggested Future Studies}

Studies of the reproducible control of porosity of aerogel materials has perhaps received the least attention of all focused topics from the aerogel community. Also, the effects of porosity on thermal conductivity have been known just as a level of a common sense, with insufficient details to add understanding and to guide development. This section outlines some additional work that could be performed for advancement of aerogel and sol-gel technologies.

- Further exploration of the DCM to further study unexplored parameters. Currently, the DCM has been thoroughly studied for TEOS and two combinations of acid catalysts (nitric acid - acetic acid and sulfuric acid - acetic acid). Preliminary study already showed that the DCM works for the hydrochloric acid - acetic acid combination as a hydrolysis catalyst. Further studies are recommended to deepen understanding of the processes and reaction mechanisms for this hydrochloric acid - acetic acid combination. These studies might include observations of changes in $\mathrm{pH}$ and temperature when the catalyzed reaction proceeds. Use of TMOS instead of TEOS as a precursor can be investigated while quantifying the amounts of catalysts, water to TMOS ratio, and hydrolysis time. Also, the pot life time of the produced sol can be prolonged by further investigation into adjusting the quantity of water used for the pre-hydrolysis step and the storage condition. A study of Small-angle X-ray scattering (SAXS) for the reaction route of the DCM can be additionally performed as it aids in better understanding the mechanisms in the sol-gel process where multiple reactions occur simultaneously.

- Further investigation on a broader range of target density for silica aerogel production process. In Chapter 4, the range of target density studied for the produced silica aerogel was arbitrarily selected and porosity change was observed. More observation can be made outside of the target density range studied by changing the process parameters such as 
solvent type and catalysts type for the DCM. This study at a broader range of target density is expected to show repetition of the oscillating manner of change in skeletal density (Figure 4-10 in Chapter 4). Results from this suggested study can provide a clearer view on how porosity is affected by target densities at a broader range and can aid in discovering some unknown relationships between porosity and two densities (apparent density and skeletal density). As the study in Chapter 4 was pursued only for TEOS as a precursor, use of TMOS, another typical precursor, can be further studied by the same experimental method.

- Further investigation on the production process for yttria and organic aerogels. Studies of porosity change resulting from changes in the target density for both types of aerogels were introduced in Chapter 5. Based on these results, more exploration of the process parameters can be imagined, such as solvent type, catalyst type, and the amount of catalyst. Changes in these parameters are expected to influence porosity, as was reported from the study on silica aerogel in Chapter 4. A broader range of target density and other precursors can also be selected for further studies. Yttrium nitrate can be used as a precursor for yttria aerogels, and combinations of resorcinol-furfuryl alcohol and phloroglucinol-furfuryl alcohol can replace that of phloroglucinol-formaldehyde in the production process for organic aerogels. Finally, as heating was required to induce gelation in synthesized organic aerogel, the heating temperature can also be varied to observe any effect on porosity.

- Effects of supercritical drying process on porosity of aerogels. All studies of the production processes for the aerogels in this work were pursued under the same condition of supercritical drying. As monolithicity of aerogel is affected by this supercritical drying step, changes in temperature and pressure of the supercritical drying can be further studied to observe how porosity is affected. The pressurization rate, depressurization rate can be 
also explored as collapse of nano pores occurs often time in these steps and thus the apparent density is changed.

- Implanting a wire set into various monolithic aerogels. In Chapter 6 , the $3 \varpi$ measurement was pursued for 3 silica aerogels and 2 organic aerogels. A much larger sample size for both aerogels can be prepared with a broad range of target density, and thermal conductivity measurements can be used to ensure the relationship proposed between thermal conductivity and porosity. Further research also can be focused on development a technique to implant a wire set into monolithic yttria aerogel and possibly other aerogel species. Tests of various types of metal wires as the heating/sensing source can also be conducted in fabricating the wire set as the electric resistance of the wire is a key parameter for the $3 \varpi$ measurement. 


\section{References}

[1] J. Kim, "Synthesis and Characterization of Nickel-Alumina Aerogel Catalysts for Carbon Dioxide Reforming of Methane," Seoul, 1998.

[2] S. Sakka and K. Kamiya, "Glasses from metal alcoholates," J.Non Cryst.Solids, vol. 42, no. 1-3, pp. 403-421, 1980.

[3] E. M. Rabinovich, "Preparation of glass by sintering," J.Mater.Sci., vol. 20, no. 12, pp. 4259-4297, 1985.

[4] D. WOOD, "Preparation of High-Silica Glasses from Colloidal Gels: III, Infrared Spectrophotometric Studies," J Am Ceram Soc, vol. 66, no. 10, pp. 693-699, 1983.

[5] D. Segal, "Sol-gel processing: Routes to oxide ceramics using colloidal dispersions of hydrous oxides and alkoxide intermediates," J. of Non-Cryst. Solids, vol. 63, no. 1, pp. 183-191, 1984.

[6] N. Maliavski, M. Guglielmi, J. Mackenzie and D. Ulrich, "Ultrastructure processing of advanced ceramics," in Third Int. Conf.. On Ultrastucture processing of ceramics, glasses and composites, San Diego, 1987.

[7] A. Gash, J. Satcher Jr. and R. Simpson, "Monolithic nickel(II)-based aerogels using an oganic epoxide: the importance of the counterion," Journal of Non-Crystalline Solids, vol. 350, pp. 145-151, 2004.

[8] C. J. Brinker and G. W. Scherer, Sol-Gel Science: The Physics and Chemistry of Sol-Gel Processing, New York: Academic Press, 1990, p. 127.

[9] M. Voronkov, V. Mileshkevich and Y. Yuzhelevski, The Siloxane Bond, New York: Consultants Bureau, 1978.

[10] A. Craievich, M. Aegerter, D. dos Santos, T. Woignier and J. Zaezycki, "A SAXS study of silica aerogels," Journal of Non-Crystalline Solids, vol. 86, no. 3, pp. 394-406, 1986.

[11] "SAXS structural study of xerogels and aerogels formed from small-molecule organic gelators," J. Phys.: Conf. Ser., vol. 100, pp. 1-4, 2008.

[12] A. Emmerling, R. Petricevic, A. Beck, P. Wang, H. Scheller and J. Fricke, "Relationship between optical transparency and nanostructural features of silica aerogels," Journal of Non-Crystalline Solids, vol. 185, pp. 240-248, 1995. 
[13] H. E. Rassy and A. Pierre, "NMR and IR spectroscopy of silica aerogels with different hydrophobic characteristics," Journal of Non-Crystalline Solids, vol. 351, no. 19-20, pp. 1603-1610, 2005.

[14] S. Zhao, B. Jiang, T. Maeder, P. Muralt, N. Kim, S. Matam, E. Jeong, Y.-L. Han and M. Kobel, "Dimensional and Structural Control of Silica Aerogel Membranes for Miniaturized Motionless Gas Pumps," ACS Appl. Mater. Interfaces, vol. 7, no. 33, p. 18803-18814, 2015.

[15] J. Sonn, "Synthesis and Characterization of Silica Aerogel," MS Thesis, Seoul, 1998.

[16] A. C. Pierre and P. M. Pajonk, "Chemistry of aerogels and their applications," Chemical Reviews, vol. 102, no. 11, pp. 4243-4265, 2002.

[17] G. Pajonk, A. Venkateswara Rao, N. Pinto, F. Ehrburrger-Dolle and M. Bellido Gil, "Monolithic carbon aerogels for fuel cell electrodes," in Proceedings of the 7th International Symposium on Scientific Bases for the Preparation of Heterogeneous Catalysts, Louvain-la-Neuve, 1998.

[18] M. Mukhopadhyay and B. Rao, "Modeling of supercritical drying of ethanol-soaked silica aerogels with carbon dioxide," Journal of Chemical Technology and Biotechnology, vol. 83, no. 8, p. 1101-1109, 2008.

[19] G. Rogacki and P. Wawrzyniak, "Diffusion of ethanol-liquid carbon dioxide in silica aerogel," Journal of Non-Crystalline Solids, vol. 186, pp. 73-77, 1995.

[20] Z. Novak and Z. Knez, "Diffusion of methanol-liquid carbon dioxide and methanolsupercritical carbon dioxide," Journal of Non-Crystalline Solids, vol. 221, pp. 163-169, 1997.

[21] R. Sadus, "Calculating critical transitions of fluid mixtures: Theory vs. experiment," AIChE Journal, vol. 40, no. 8, p. 1376-1403, 1994.

[22] S. Kistler, "Coherent Expanded Aerogels and Jellies," Nature, vol. 127, p. 741, 1931.

[23] A. Hunt and A. Chen, "Aerogel: Energy-Efficient Material for Buildings," Lawrence Berkeley National Laboratory, 1995. [Online]. Available: https://eetd.lbl.gov/newsletter/cbs_nl/nl08/cbs-nl8-aerogel.html.

[24] M. Ayers, "The Early Days of Aerogels," AEOGEL.ORG, 2000. [Online]. Available: http://www.aerogel.org/?p=416.

[25] S. J. Teichner, J. Moutel, F. Bonnin, D. Roussel, P. Mestreau, R. Jouan, L. Koch, M. Casse and M. Cantin, "SILICA AEROGELS USED AS CHERENKOV RADIATORS," Nuclear Instruments and Methods, vol. 118, no. 1, pp. 177-182, 1974. 
[26] A. H. a. K. L. P.H. Tewari, "Ambient-temperature supercritical drying of transparent silica aerogel," Material Letter, vol. 3, pp. 363-367, 1985.

[27] G. Kim, S. Hyun, S. Hwang and G. Kim, "Cost-effective synthesis of silica aerogels from waterglass/teos by ambient drying and their applications," Ceramic Engineering and Science Proceedings, vol. 23, no. 4, pp. 73-78, 2002.

[28] M. Seyedjalali and M. R. Madani, "Silica aerogel thick film, an alternative to micromachined air gap for thermal insulation," Electronics Letters, vol. 51, no. 11, pp. 849-850, 2015.

[29] G. Kim and S. Hyun, "Synthesis of window glazing coated with silica aerogel films via ambient drying," Journal of Non-Crystalline Solids, vol. 320, no. 1-3, pp. 125132, 2003.

[30] C. Wang, C. Wu, I. Chen and Y. Huang, "Humidity sensors based on silica nanoparticle aerogel thin films," Sensors and Actuators, B: Chemical, vol. 107, no. 1, pp. 402-410, 2005.

[31] S. Hyun, J. Kim and H. Park, "Synthesis and characterization of low-dielectric silica aerogel films," Journal of the American Ceramic Societ, vol. 83, no. 3, pp. 533$540,2000$.

[32] G. Kim, S. Hyun, S. Hwang and G. Kim, "Cost-effective synthesis of silica aerogels from waterglass/teos by ambient drying and their applications," in Ceramic Engineering and Science Proceedings, 2002.

[33] G. Kim and S. Hyun, "Synthesis and characterization of silica aerogel films for intermetal dielectrics via ambient drying," Thin Solid Films, vol. 460, no. 1-2, pp. 190-200, 2004.

[34] M. Aegerter, N. Leventis and M. Koebel, "Recent aerogel development," in Aerogels Handbook, 2011, pp. 11-12.

[35] T. Roth, A. Anderson and M. Carroll, "Analysis of a rapid supercritical extraction aerogel fabrication: Prediction of thermodynamic conditions during processing," J. Non-Cryst Solids, vol. 354, p. 3685-3693, 2008.

[36] K. H. Bangi Uzma, H. Hirashima, V. RaO and P. Rao, "Physico-chemical properties of ambiently dried sodium silicate based aerogels catalyzed with various acids," J Sol-Gel Sci Technol, vol. 50, pp. 87-97, 2009.

[37] T. F. Baumann, S. O. Kucheyev, A. E. Gash and J. H. Satcher Jr., "Facile synthesis of a crystalline, high-surface area SnO2 aerogel," Advanced Mater , vol. 17, p. 546$1548,2005$. 
[38] C. N. Chervin, B. J. Clapsaddle, H. W. Chiu, A. E. Gash, J. H. Satcher Jr. and S. M. Kauzlarich, "Aerogel Synthesis of Yttria-Stabilized Zirconia by a NonAlkoxide Sol-Gel Route," Chem Mater 17:, vol. 17, p. 3345-3351, 2005.

[39] A. E. Gash, M. Pantoya, J. H. Satcher and R. L. Simpson, "Nanostructured energetic materials: aerogel thermite composites," in American Chemical Society, Division of Polymer Chemistry, 2008.

[40] J. F. Hund, M. F. Bertino, G. Zhang and C. Sotiriou-Leventis, "Synthesis of homogeneous alloy metal nanoparticles in silica aerogels," J Non-Cryst Solids, vol. 350, pp. 9-13, 2004.

[41] M. F. Bertino, R. R. Gadipalli, J. G. Story JG, C. G. Williams, G. Zhang , C. SotiriouLeventis, A. T. Tokuhiro, S. Guha and N. Leventis, "Laser writing of semiconductor nanoparticles and quantum dots," Appl Phys Letters, vol. 85, p. 6007-6009, 2004.

[42] N. Kuthirummal, A. Dean, C. Yao and W. Risen, "Photo-formation of gold nanoparticles: Photoacoustic studies on solid monoliths of Au(III)-chitosansilica aerogels Spectrochim Acta A," Mol Biomol Spectroscopy, vol. 70A, pp. 700-703, 2008.

[43] F. Fischer, A. Rigacci, R. Pirard and S. A. Berthon-Fabry, "Cellulose-based aerogels," Polymer, vol. 47, p. 7636-7645, 2006.

[44] H. Luo, G. Churu, E. F. Fabrizio, J. Schnobrich, A. Hobbs, A. Dass, S. Mulik, Y. Zhang, B. P. Grady and A. Capecelatro, "Synthesis and characterization of the physical, chemical and mechanical properties of isocyanate-crosslinked," J Sol-Gel Sci Technol, vol. 48, pp. 113-134, 2008.

[45] N. Leventis, N. Chandrasekaran, A. Sadekar, C. Sotiriou-Leventis and H. Lu, "One-Pot Synthesis of Interpenetrating Inorganic/Organic Networks of $\mathrm{CuO/Resorcinol-Formaldehyde} \mathrm{Aerogels:} \mathrm{Nanostructured} \mathrm{Energetic}$ Materials," J Amer Chem Soc, vol. 131, p. 4576-4577, 2009.

[46] U. Bauer, M. Darsillo, R. Field, J. Floess, J. Frundt, S. Rouanet and D. Doshi, "Aerogel particles and methods of making same". Int. Appl Patent WO 2008115812 A2 20080925, 2008.

[47] "Nano High-Tech Co., Ltd.," Nano High-Tech, April 2004. [Online]. Available: http://aerogel.company.weiku.com. [Accessed 62 2017].

[48] "OKAGEL," OKALUX, 2008. [Online]. Available: http://www.okaluxna.com/products/okagel. 
[49] D. Albert, G. Andrews and J. Bruno, "Organic, open cell foam materials, their carbonized derivatives and methods for production". U.S. Pat. Appl. Patent US 2002064642, 2002.

[50] M. Augustyniak, K. Hamilton and H. Kalkstein, "Manufacture of architectural membrane structures based on aerogel-containing 3-ply composites". U.S. Pat. Appl. Patent US 2008229704 A1 20080925, 2008.

[51] J. Lee, G. Gould and W. Rhine, "Polyurea based aerogel for a high performance thermal insulation material," J Sol-Gel Sci Technol, vol. 49, p. 209-220, 2009.

[52] F. Shi, L. Wang and J. Liu, "Synthesis and characterization of silica aerogels by a novel fast ambient pressure drying process," Materials Letters, vol. 60, no. 29-30, pp. 3718-3722, 2006.

[53] L. Durães, T. Matias, R. Patrício and A. Portugal, "Silica based aerogel-like materials obtained by quick microwave drying," Materialwissenschaft und Werkstofftechnik, vol. 44, no. 5, pp. 380-385, 2013.

[54] C. Daitch, J. Brenizer, B. Hosticka, L. Mason, P. M. Norris, M. Luo and L. DeLucas, "Smart Aerogel". USA Patent 6,447,991, 1092002.

[55] E. Cohen, "Thermal Properties of Advanced Aerogel Insulation," in M.S. Thesis in Mechanical Engineering, Cambridge, Massachusetts Institute of Technology, 2011.

[56] R. Yokokawa, J.-A. Paik, B. Dunn, N. Kitazawa, H. Kotera and C.-J. Kim, "Mechanical properties of aerogel-like thin films used for MEMS," J. of Micromechanics and Microengineering, vol. 14, pp. 681-686, 2004.

[57] M. L. Bauer, C. M. Bauer, M. C. Fish, R. E. Matthews, G. T. Garner, A. W. Litchenberger and P. M. Noris, "Thin-film aerogel thermal conductivity measurements via 3omega," J. of Non-Crystalline Solids, vol. 357, no. 15, pp. 2960-2965, 2011.

[58] M. Schmidt and F. Schwertfeger, "Applications for silica aerogel products," Journal of Non-Crystalline Solids, vol. 225, pp. 364-368, 1998.

[59] B. Daly, G. Antonellia, H. Marisa, W. Fordb, L. Wongb and E. Andideh, "Measurements of the thermal conductivity of amorphous materials with low dielectric constants.," Physica B: Condensed Matter, Vols. 316-317, pp. 24-257, 2002.

[60] D. Cahill, J. Olson, H. Fischer, S. Watson, R. Stephens, R. Tait, T. Ashworth and R. Pohl, "Thermal conductivity and specific heat of glass ceramics.," Physical Review B, Vols. 44-22, pp. 12226-32, 1991.

[61] D. Cahill and R. Pohl, "Heat flow and lattice vibrations in glasses.," Solid State. Communications, vol. 70, pp. 927-930, 1989. 
[62] P. Hopkins and T. Beechem, "Phonon Scattering and Velocity Considerations in the Minimum Phonon Thermal Conductivity of Layered Solids above the Plateau," Nanoscale and Microscale Thermophysical Engineering, vol. 14, pp. 51-61, 2010.

[63] M. Kaviany, Principles of Heat Transfer, New York: John Wiley \& Sons, 2002, pp. 193194.

[64] R. Zeller and R. Pohl, "Thermal Conductivity and Specific Heat of Noncrystalline Solids," Phys. Rev. B, vol. 4, no. 6, pp. 2029-2041, 1971.

[65] P. Hopkins and E. Piekos, "Lower limit to phonon thermal conductivity of disordered, layered solids," Applied Physics Letters, vol. 94, pp. 181901-1-3, 2009.

[66] D. Nadargi, S. Latthe and A. Venkateswara Rao, "Effect of post-treatment (gel aging) on the properties of methyltrimethoxysilane based silica aerogels prepared by two-step sol-gel process," J Sol-Gel Sci Technol, vol. 49, pp. 53-59, 2009.

[67] H. Nörenberg, V. Burlakov, H.-J. Kosmella and Y. Tsukahara, "Pressure-dependent permeation of noble gases ( $\mathrm{He}, \mathrm{Ne}, \mathrm{Ar}, \mathrm{Kr}, \mathrm{Xe}$ ) through thin membranes of oriented polypropylene (OPP) studied by mass spectrometry," Polymer, vol. 42, pp. 10021-10026, 2001.

[68] G. Joos and I. Freeman, Theoretical Physics, Mineola: Dover Publications, 1953.

[69] J. Slater, "Atomic Radii in Crystals," J. Chem. Phys., vol. 41, no. 10, pp. 3199-3204, 1964.

[70] E. Clementi, D. Raimondi and W. Reinhardt, "Atomic Screening Constants from SCF Functions. II. Atoms with 37 to 86 Electrons.," J. Chem. Phys., vol. 47, no. 4, pp. 1300-1307, 1967.

[71] G. Walrafen, M. Hokmabadi, N. Holmes, W. Nellis and S. Henning, "Raman spectrum and structure of silica aerogel," J. Chemistry Physics, vol. 82, no. 5, pp. 24722476, 1985.

[72] E. Keng, "Air and Helium Pycnometer," Powder Technology, vol. 3, no. 3, pp. 179-180, 1968.

[73] R. M. Costescu,, A. J. Bullen, G. Matamis, K. E. O’Hara and D. G. Cahill, " Thermal conductivity and sound velocities of hydrogen-silsesquioxane low-k dielectrics," Pysical Review B., vol. 65, p. 094205, 2002.

[74] A. Einstein, "Eine Beziehung zwischen dem elastischen Verhalten und der spezifischen Wärme bei festen Körpern mit einatomigem Molekül," Ann. Phys. (Leipzig), vol. 340, no. 9, pp. 679-694, 1911. 
[75] D. Stroud, "The effective medium approximations: Some recent developments," Superlattices and Microstructures, vol. 23, no. 3/4, pp. 567-574, 1998.

[76] E. A., "Thermal Conductivity of Ceramic Refractory Materials, Calculation from Thermal Conductivity of Constituents.," Ceramic Abstracts, vol. 11, pp. 353360, 1932.

[77] R. Landauer, "The electrical resistance of binary metallic mixtures," J. Appl. Phys., vol. 23, no. 7, pp. 779-784, 1952.

[78] P. N. Sen, C. Scala and M. H. Cohen, "A self-similar model for sedimentary rocks with application to the dielectric constant of fused glass beads," Geophysics, vol. 46, pp. 781-795, 1981.

[79] M. L. Cleary, J. R. Haynes, E. A. Schon and J. B. Lingrel, "Identification by nucleotide sequence analysis of a goat pseudoglobin gene," Nucleic Acids Research, vol. 8, no. 20, p. 4791-4802, 1980.

[80] A. Norris, "A differential scheme for the effective moduli of composites," Mechanics of Materials, vol. 4, pp. 1-16, 1985.

[81] J. B. Walsh , "Static deformation of rock," J. Eng. Mech. Div. ASCE, vol. 106, pp. 10051019, 1980.

[82] M. Avellaneda, "Iterated homogenization, differential effective medium theory and applications," Commun. Pure Appl. Math, vol. 10, pp. 527-554, 1987.

[83] X. Lu, O. Nilsson, J. Fricke and R. Pekala, "Thermal and electrical conductivity of monolithic carbon aerogels," J. Appl. Phys, vol. 73, pp. 581-584, 1993.

[84] D. Cahill, "Thermal conductivity measurement from 30 to $750 \mathrm{~K}$ : the $3 \omega$ method," Review of Scientific Instruments, vol. 61, pp. 802-808, 1990.

[85] D. Cahill, R. Tait, R. Stephens, S. Watson and R. Pohl, "in Thermal Conductivity," in Themal Conductivity 21, New York, 1990.

[86] C. Hu, M. Morgan, P. S. Ho, A. Jain, W. N. Gill, J. L. Plawsky and P. C. Wayner, Jr., "Thermal conductivity study of porous low-k dielectric materials," Appl. Phys. Lett., vol. 77, no. 1, pp. 145-147, 2000.

[87] J. Fricke, X. Lu, P. Wang, D. Buttner and U. Heinemann, "Optimization of monolithic silica aerogel insulants," Int. J. Heat Mass Transf, vol. 35, pp. 2305-2309, 1992.

[88] P. E. Hopkins, B. Kaehr, E. S. Piekos, D. Dunphy and C. J. Brinker, "Minimum thermal conductivity considerations in aerogel thin films," J. Appl. Phys., vol. 111, p. 113532, 2012. 
[89] D. G. Cahill, S. K. Watson and R. O. Pohl, "Lower limit to the thermal conductivity of disordered crystals," Phys. Rev. B, vol. 46, p. 6131, 1992.

[90] J. Gross, J. Fricke, R. W. Pekala, and L. W. Hrube, "Elastic nonlinearity of aerogels," Phys. Rev. B, vol. 45, p. 12774, 1992.

[91] S. Spagnol, B. Lartigue and A. Trombe, "Experimental Investigations on the Thermal Conductivity of Silica Aerogels by a Guarded Thin-Film-Heater Method," J. Heat Transfer, vol. 131, no. 7, p. 074501, 2009.

[92] T. Wei, S. Lu and Y. Chang, "A New Class of Opacified Monolithic Aerogels of Ultralow High-Temperature Thermal Conductivities," J. Phys. Chem. C 113, vol. 113, pp. 7424-7428, 2009.

[93] O. Nilsson, A. Fransson and O. Sandberg, "Thermal Properties of Silica Aerogel," in Fricke J. (eds) Aerogels. Springer Proceedings in Physics, Berlin, 1986.

[94] G. Wei, Y. Liu, X. Zhang, F. Yu and X. Du, "Thermal conductivities study on silica aerogel and its composite insulation materials," International Journal of Heat and Mass Transfer, vol. 54, no. 11-12, pp. 2355-2366, 2011.

[95] S. Zeng, A. Hunt and R. Greif, "Geometric structure and thermal conductivity of porous medium silica aerogel," J. Heat Transfer, vol. 117, no. 4, pp. 10551058, 1995.

[96] I. Sumirat, Y. Ando and S. Shimamura, "Theoretical consideration of the effect of porosity on thermal conductivity of porous materials," J Porous Mater, vol. 13, pp. 439-443, 2006.

[97] A. Jain, S. Rogojevic, S. Ponoth, W. N. Gill, J. L. Plawsky, E. Simonyi and S.-T. Chen, "Processing dependent thermal conductivity of nanoporous silica xerogel films," J. Appl. Phys., vol. 91, no. 5, p. 3275, 2002.

[98] J. Liu, D. Gan, C. Hu, M. Kiene, P. S. Ho and W. Volks, "Porosity effect on the dielectric constant and thermomechanical properties of organosilicate films," Appl. Phys. Lett. 81, 4180 (2002); vol. 81, no. 22, pp. 4180-4182, 2002.

[99] L. W. Hrubesh and R. W. Pekala, "Thermal properties of organic and inorganic aerogels," Journal of Materials Research, vol. 9, no. 3, pp. 731-738, 1994.

[100] J. Wang, J. Kuhn and X. Lu, "Monolithic silica aerogel insulation doped with TiO2 powder and ceramic fibers," Journal of Non-Crystalline Solids, vol. 186, p. 296-300, 1995.

[101] J. Kuhn, G. Gleissner, M. Arduini-Schuster, S. Korder and J. Fricke, "Integration of mineral powders into SiO2 aerogels," Journal of Non-Crystalline Solids, vol. 186, pp. 291-295, 1995. 
[102] S. Zeng, A. Hunt and R. Greif, "Theoretical modeling of carbon content to minimize heat transfer in silica aerogel," Journal of Non-Crystalline Solids, vol. 186, pp. 271-277, 1995.

[103] Y. G. Kwon, S. Y. Choi, E. S. Kang and S. S. Baek, "Ambient-dried silica aerogel doped with TiO2 powder for thermal insulation," J. Mater. Sci., vol. 35, no. 24, pp. 6075-6079, 2000.

[104] P. Sarawade, J.-K. Kim, H.-K. Kim and H.-T. Kim, "High specific surface area TEOSbased aerogels with large pore volume prepared at an ambient pressure," Appl Surf Sci, vol. 254, pp. 574-579, 2007.

[105] P. Hiemenz, Principles of colloid and surface chemistry, New York: Marcel Dekker, 1977.

[106] A. Rao, M. Kulkarni and D. Amal, "Superhydrophobic silica aerogels based on methyltrimethoxysilane precursor," J Non-Cryst Solids, vol. 330, no. 1, pp. 187-195, 2003.

[107] S. Clark, "Aerogel Market by Raw Material (Silica, Carbon, Alumina), Form (Blanket, Particle/Powder, Block, Panel) and Application (Building insulation, Oil \& Gas, Apparel, Electronics) - Global Opportunity Analysis and Industry Forecast, 2014 - 2021," Allied Market Research, San Francisco, 2016.

[108] F. Hoffmann and M. Fröba, "Vitalising porous inorganic silica networks with organic functions-PMOs and related hybrid materials," Royal Society of Chemistry, vol. 40, pp. 608-620, 2011.

[109] T. Tillotson and L. Hurbesh, "Transparent ultralow-density silica aerogels prepared by a two-step sol-gel process," J. of Non-Crystalline Solids, vol. 145, pp. 44-50, 1992.

[110] N. Hall and J. Conant, "A Study of Superacid Solutions," Journal of the American Chemical Society, vol. 49, no. 12, p. 3062-3070, 1927.

[111] "Evonik/ Dynasilan SILBOND," EVONIK, 2017. [Online]. Available: https://www.dynasylan.com/product/dynasylan/en/products/productgroups/dynasylan-silbond/Pages/default.aspx. [Accessed 17 April 2017].

[112] S. Brunauer, P. Emmett and E. Teller, "Adsorption of Gases in Multimolecular Layers," J. Am. Chem. Soc, vol. 60, no. 2, pp. 309-319, 1938.

[113] Micromeritics ASAP 2010 operator's mannual V3.00, Norcross: Micromeritics, 1996.

[114] C. Lastoskie, K. Gubbin and N. Quirke, "Pore Size Distribution Analysis of Microporous Carbons: A Density Functional Theory Approach," J. Phys. Chem, vol. 97, pp. 4786-4796, 1993. 
[115] S. Lee, C. Park and S. Kim, "Temperature-Sensitive Sol-Gel Transition Behavior of Biodegradable Four-Arm Star-Shaped PEG-PLGA Block Copolymer Aqueous Solution," Polymer Journal, vol. 41, no. 5, pp. 425-431, 2009.

[116] D. Schiff, "AEROGEL INSULATED PANE," US Dept. of Energy, 185 2016. [Online]. Available: https://arpa-e.energy.gov/?q=slick-sheet-project/aerogelinsulated-pane. [Accessed 113 2017].

[117] S. Stecura, "Two-layer thermal coating for high temperature componets," in American Ceramic Society, Joint Fall Meeting of the Basic Science, San Francisco, 1977.

[118] J. Zhang, Z. Zhang, Z. Tang, Y. Lin and Z. Zheng, "Luminescent properties of yttia:Eu synthesized by sol-gel processing," J. Mater. Process. Technol., vol. 121, no. 23, pp. 265-268, 2002.

[119] W.-W. Zhang, W.-P. Zhang, P.-B. Xie, M. Yin, H. Chen, L. Jing, Y.-S. Zhang, L.-R. Lou and S.-D. Xia, "Optical properties of nanocrystalline Y203:Eu depending on its odd structure," J. Colloid Interf. Sci., vol. 262, no. 2, pp. 588-593, 2003.

[120] P. Sharma, JilaviM.H., R. Nass and H. Schmidt, "Tailoring the particle size from $\mu \mathrm{m} \rightarrow \mathrm{nm}$ scale by using a surface modifier and their size effect on the fluorescence properties of europium doped yttria," J. Lumin, vol. 82, no. 3, pp. 187-193, 1999.

[121] J. Eid, A. Pierr and G. Baret, "Preparation and characterization of transparent Eu doped Yttria aerogel monoliths, for application in luminescence," Journal of Non-Crystalline Solids, vol. 351, p. 218-227, 2005.

[122] R. Pekala and F. Kong, "A SYNTHETIC ROUTE TO ORGANIC AEROGELS MECHANISM, STRUCTURE, AND PROPERTIES," Le Journal de Physique Colloques, vol. 50, no. C4, pp. 33-40, 1989.

[123] R. Pekala, C. Alviso, X. Lu, J. Gross and J. Fricke, "New organic aerogels based upon a phenolic-furfural reaction," Journal of Non-Crystalline Solids, vol. 188, no. 1-2, pp. 34-40, 1995.

[124] A. Stark, "Advanced carbon aerogels for energy applications," Lawrence Livermore National Laboratory, 14 March 2011. [Online]. Available: https://www.llnl.gov/news/advanced-carbon-aerogels-energy-applications. [Accessed 1 March 2017].

[125] H.-J. Kim, W.-I. Kim, T.-J. Park, H.-S. Park and D. Suh, "Highly dispersed platinumcarbon aerogel catalyst for polymer electrolyte membrane fuel cells," Carbon, vol. 46, no. 11, pp. 1393-1400, 2008. 
[126] H. Chen, E. Hollinger, Y. Wang and D. Schiraldi, "Facile fabrication of poly(vinyl alcohol) gels and derivative aerogels.," Polymer, vol. 55, pp. 380-384, 2014.

[127] R. Li, C. Chen, J. Li, L. Xu, G. Xiao and D. Yan, "A facile approach to superhydrophobic and superoleophilic graphene/polymer aerogels.," J. Mater. Chem. A, vol. 2, p. 3057-3064, 2014.

[128] Q. Zheng, Z. Cai and S. Gong, "Green synthesis of polyvinyl alcohol (PVA)-cellulose nanofibril (CNF) hybrid aerogels and their use as superabsorbents.," J. Mater. Chem. A, vol. 2, p. 3110-3118, 2014.

[129] A. Donius, A. Liu, L. Berglund and U. Wegst, "Superior mechanical performance of highly porous, anisotropic nanocellulose-montmorillonite aerogels prepared by freeze casting.," J. Mech. Behav. Biomed. Mater., vol. 37, p. 88-99, 2014.

[130] B. Wicklein, A. Kocjan, G. Salazar-Alvarez, F. Carosio, G. Camino, M. Antonietti and L. Bergström, "Thermally insulating and fire-retardant lightweight anisotropic foams based on nanocellulose and graphene oxide.," Nat. Nanotechnol., vol. 10, p. 277-283, 2014.

[131] H. Chen, Y. Wang and D. Schiraldi, "Preparation and flammability of poly(vinyl alcohol) composite aerogels.," ACS Appl. Mater. Interfaces, vol. 6, p. 67906796, 2014.

[132] H. Chen, B. Liu, W. Huang, J. Wang, G. Zeng, W. Wu and D. Schiraldi, "Fabrication and properties of irradiation-cross-linked poly(vinyl alcohol)/clay aerogel composites.," ACS Appl. Mater. Interfaces, vol. 6, p. 16227-16236, 2014.

[133] Q. Zheng, A. Javadi, R. Sabo, Z. Cai and S. Gong, "Polyvinyl alcohol (PVA)-cellulose nanofibril (CNF)-multiwalled carbon nanotube (MWCNT) hybrid organic aerogels with superior mechanical properties.," RSC Adv., vol. 3, p. 2081620823, 2013.

[134] G. Maciel, S. Chuang and L. Gollob, "Solid-state carbon-13 NMR study of resol-type phenol-formaldehyde resins," Macromolecules, vol. 17, no. 5, pp. 1081-1087, 1984.

[135] K. Barral, "Low-density organic aerogels by double-catalysed synthesis," Journal of Non-Crystalline Solids, vol. 225, p. 46-50, 1998.

[136] A. Jacquot, F. Vollmer, B. Bayer, M. Jaegle, D. Ebling and H. Böttner, "Thermal Conductivity Measurements on Challenging Samples by the 3 Omega Method," J. of Electronic Materials, vol. 39, no. 9, pp. 1621-1626, 2010.

[137] S. Lee and D. Cahill, "Heat transport in thin dielectric films," J. of Applied Physics, vol. 81, no. 6, pp. 2590-2595, 1997. 
[138] T. Borca-Tasciuc, A. Kumar and G. Chen, "Data reduction in $3 \omega$ method for thin-film thermal conductivity determination," Review of Scientific Instruments, vol. 72, no. 4, pp. 2139-2147, 2001.

[139] V. Giraud, J. Cluzel, V. Sousa, A. Jacquot, A. Dauscher, B. Leoir, H. Scherrer and S. Romer, "Thermal characterization and analysis of phase change random access memory," J. of Applied Physics, vol. 98, no. 1, pp. 13520-1-7, 2005.

[140] M. Bauer, Nanostructure dictating thermal transport in heterogeneous material systems, PhD dissertation, 2015.

[141] J. KIm, A. Feldman and D. Novotny, "Application of the three omega thermal conductivity measurement method to a film on a substrate of finite thickness.," J. Appl. Phys., vol. 86, no. 7, pp. 3959-63, 1999.

[142] N. Birge, "Specific-heat spectroscopy of glycerol and propylene glycol near the glass transition," PHYSICAL REVIE\% 8, vol. 34, no. 3, pp. 1631-1642, 1986.

[143] X. Hu, A. Padilla, J. Xu, T. Fisher and K. Goodson, "3-Omega Measurements of Vertically Oriented Carbon Nanotubes on Silicon," Journal of Heat Transfer, vol. 128, pp. 11009-1113, 2006.

[144] H. Carslaw and J. Jaeger, Conduction of heat in solids, 2nd ed, Oxfod: Clarendon Press, 1959.

[145] E. Rezaei and J. Moghaddas, "Thermal conductivities of silica aerogel composite insulating material," Advanced Materials Letters, vol. 7, no. 4, pp. 296-301, 2016.

[146] R. Caps and J. Fricke, Caps, R., and Fricke, J., 2004, "Aerogels for Thermal Insulation," Sol-Gel Technologies for Glass Producers and Users, , eds., , Boston, pp. 349353. [CrossRef], M. a. M. M. A.Aegerter, Ed., Boston: Kluwer Academic, 2004.

[147] E. Cohen and L. Glicksman, "Thermal Properties of Silica Aerogel Formula," Journal of Heat Transfer, vol. 137, no. 8, pp. 081601-081608, 2015.

[148] X. Lu, M. Arduini-Schuster, J. Kuhn, O. Nilsson, J. Fricke and R. Pekala, "Thermal conductivity of monolithic organic aerogels.," Sceince, vol. 255, no. 5047, pp. 971-972, 1992. 